Entering Through The Green Door: Exploring the Value of 'Fresh'

by

Rebecca Warden

A thesis submitted to the Faculty of Graduate and Postdoctoral Affairs in partial fulfillment of the requirements for the degree of

Master of Arts

in

Sociology

Carleton University

Ottawa, Ontario

(C) 2011 
Library and Archives

Canada

Published Heritage Branch

395 Wellington Street Ottawa ON K1A ON4 Canada
Bibliothèque et

Archives Canada

Direction du

Patrimoine de l'édition

395, rue Wellington

Ottawa ON K1A ON4

Canada
Your file Votre référence

ISBN: 978-0-494-83100-7

Our file Notre référence

ISBN: 978-0-494-83100-7
NOTICE:

The author has granted a nonexclusive license allowing Library and Archives Canada to reproduce, publish, archive, preserve, conserve, communicate to the public by telecommunication or on the Internet, loan, distribute and sell theses worldwide, for commercial or noncommercial purposes, in microform, paper, electronic and/or any other formats.

The author retains copyright ownership and moral rights in this thesis. Neither the thesis nor substantial extracts from it may be printed or otherwise reproduced without the author's permission.
AVIS:

L'auteur a accordé une licence non exclusive permettant à la Bibliothèque et Archives Canada de reproduire, publier, archiver, sauvegarder, conserver, transmettre au public par télécommunication ou par l'Internet, prêter, distribuer et vendre des thèses partout dans le monde, à des fins commerciales ou autres, sur support microforme, papier, électronique et/ou autres formats.

L'auteur conserve la propriété du droit d'auteur et des droits moraux qui protège cette thèse. $\mathrm{Ni}$ la thèse ni des extraits substantiels de celle-ci ne doivent être imprimés ou autrement reproduits sans son autorisation.
In compliance with the Canadian Privacy Act some supporting forms may have been removed from this thesis.

While these forms may be included in the document page count, their removal does not represent any loss of content from the thesis.
Conformément à la loi canadienne sur la protection de la vie privée, quelques formulaires secondaires ont été enlevés de cette thèse.

Bien que ces formulaires aient inclus dans la pagination, il n'y aura aucun contenu manquant.

\section{Canadä}


This thesis is concerned with identifying and exploring the values that inform food choice in a contemporary urban food system. Using The Green Door Restaurant as a site of study, I demonstrate that 'fresh' has emerged as a particularly significant qualifier of 'good' food; individuals rely on a broad, overarching commitment to 'freshness' in their efforts to present a responsible, 'ethical' self. I illustrate that individuals' engagement with 'fresh' exemplifies a critical breakdown in processes of self for the urban consumer, whose efforts to present a responsible, 'ethical' self are ultimately undermined by their self-conscious recognition of inequity and privilege, and points to the limitations of market-based, consumer-choice efforts to challenge the unsustainable and inequitable practices of our contemporary Canadian food system. This study draws on data gathered through ethnographic field observation and 15 semi-structured, in-depth interviews. 
I began this work to explore a personal interest that has unwaveringly held my attention all of my life: food. Immersing myself in this project has not only allowed me to explore the sociological dimensions of what, how, and why we eat the food we do; it has also taught me innumerable lessons about myself as a researcher, writer, artist and student. I am so grateful to have had this experience and have a number of important people to thank.

First, I would like to thank all of the people who volunteered as interview participants for this project and so generously shared their time and thoughts. I sincerely valued our conversations and found it to be an absolute pleasure to speak with each of you.

I also owe great thanks to Dr. Mike Mopas for seeing me through this process. For the past two years, you have provided me with resources and facilitated discussions that have challenged me to think deeply about this topic that I love. Thank you for bringing focus to food in such exciting, intelligent ways.

I would also like to express huge gratitude to Dr. Karen March. Thank you for putting aside your (unfathomable) neutrality about food to help facilitate this project. Your passion for research and your confidence in students' abilities to go out and explore their world makes you a truly wonderful mentor.

To the members of Motivational Monday: thank you for everything - for the collaborative dinners, bike rides, road trips, yoga classes, and weekly check-ins. Being able to walk beside such a dynamic group of people as I did this work has made all the difference.

I am also incredibly grateful to my parents, Kelly \& Dave Warden, for all their support. Thank you for believing that I am capable of great things (even really horrible things that require me to sit still and focus on details for long periods of time) and encouraging me to climb big mountains.

And last, but not least, thanks to Kyle Hamilton for standing by me through these last two years of tunnel vision focus, end-of-my-rope tears, \& (subsequent) eureka-moment discoveries. Thank you for reading every word I write, providing thoughtful feedback that helps me understand even more clearly what I really mean, and always telling me I'm brilliant. 


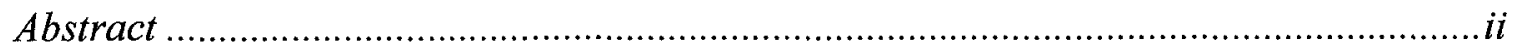

Acknowledgments .............................................................................................ii

Chapter 1 INTRODUCTION 1

Thesis Outline ........................................................ 7

Food Choices in a Distinct Style of Urban Food Consumption ...................8

Entering Through The Green Door ............................................10

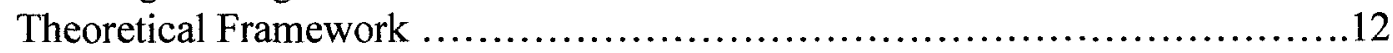

$\begin{array}{lll}\text { Chapter } 2 & \text { LITERATURE REVIEW } & 16\end{array}$

Making Food Choices .............................................................19

Making Choices: Looking at the Logic of the Regulating Self .................23

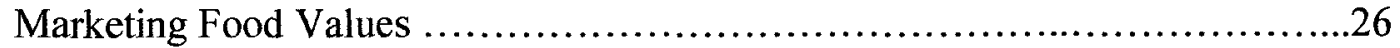

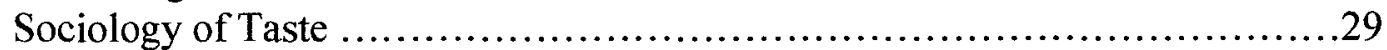

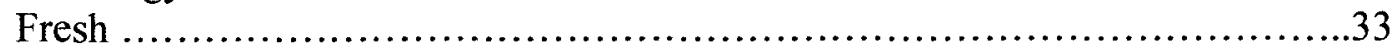

$\begin{array}{lll}\text { Chapter } 3 & \text { RESEARCH DESIGN } & 37\end{array}$

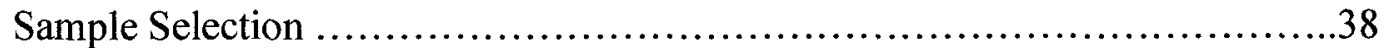

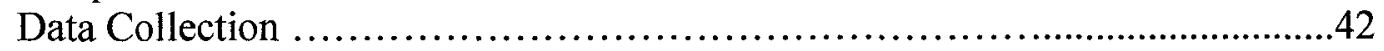

Data Analysis Strategy ................................................50

Considerations for Qualitative Research ..................................56

Chapter 4 ANALYSIS I: The Value of Fresh 60

De-constructing the Fresh Food Ethic .........................................61

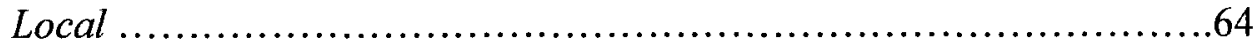

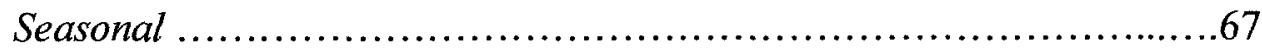

Non-Contaminated .................................................. 71

Non-Packaged/Non-Processed .....................................78

Human Handled ................................................... 83

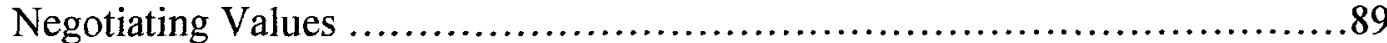

Consumption Challenges ..........................................99

Making Informed Choices to Navigate Risks ...........................94

Personal Food Practices:An Omnivorous Approach .....................99

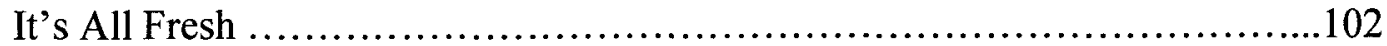

Chapter 5 ANALYSIS II: CONSTRUCTING TASTE IN A 'FRESH' SPACE 104

The Green Door Restaurant: A 'Fresh' Space ..............................107

Fresh: Democratizing ...................................................117

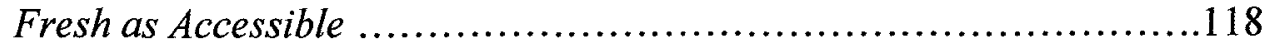

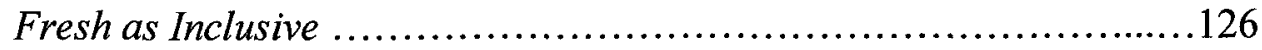

Fresh: Status \& Distinction ............................................ 131

Fresh as Exclusive ..................................................132

Food Knowledge: Evaluating Quality .................................135

Meeting Standards of Cleanliness \& Comfort ...........................140 


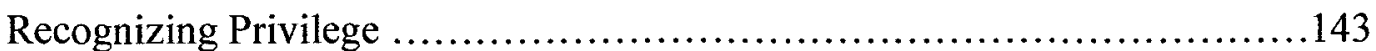

Recognizing Problems: With Privilege, Comes Responsibility ................152

"Feeling Better" by Entering Through The Green Door .......................158

Chapter 6 CONCLUSION: FINAL THOUGHTS ON 'FRESH' 164

Connecting 'Fresh' to Collective Action (vs. Individualism) .................. 166

Why Does it Matter? ...................................................... 173

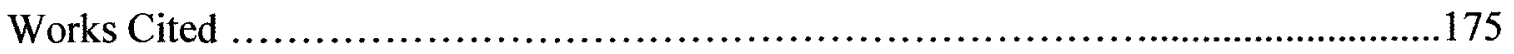


APPENDIX A: Call for Interview Participants ...................................180

APPENDIX B: Interview Script ............................................... 181

APPENDIX C: Analysis Flow Chart …........................................ 182 


\section{CHAPTER 1}

\section{INTRODUCTION}

For well-off consumers in well-off countries, choosing has become one of the hardest parts of eating. (Freidberg, 2009: 17)

Urban centers in North America rely on processes of food production, distribution, and consumption that dramatically affect how individuals experience the food they eat. In stark contrast to those residing in non-industrialized regions in the Global South, who rely predominantly on small-scale subsistence farming, many people who live in the West engage with food primarily as consumers. Industrialization and globalization have contributed to the development of a Western urban food system that effectively prevents the individual from connecting to his/her food in traditional capacities of production and preparation. Instead, urban individuals ${ }^{1}$ navigate through an incredible variety of food commodities, making dozens of value-laden choices every day, from a seemingly limitless supply of food options, about the food they eat. Whereas such luxury as choice of food is not available to the majority of the world's population, food is easily available to many people residing in westernized society, particularly those of the middle and upper class, and for these people, food choice constitutes a significant component of social life.

\footnotetext{
${ }^{1}$ I use the term 'urban individual' throughout this thesis to refer to people who have access to the variety of food commodities available in an industrialized, globalized Western city. The 'urban individual' is able to make particular food choices because those choices are readily available to him or her in a North American urban centre. While industrialization has created a climate of unprecedented variety in food commodities, it is important to note that these food choices are particularly accessible to people of the middle or upper class.
} 
Choices about food begin from the moment we wake up in the morning. Do you have time for a sit-down breakfast, or will you grab a granola bar on the way out the door? Acai Berry Medley or Fibre One? Maybe you'd be better off picking up a breakfast sandwich from Starbucks? Will you pack a lunch, or buy something from the Thai restaurant across the street? Want something even faster? Pick up a hotdog from the stand on the corner. Should it be a veggie dog today? Maybe not the bun, because you suspect you may be intolerant to gluten. How much sodium is in that mustard? Do they serve fair trade coffee at the café where you're meeting your friend? If not, will you go somewhere else? Will you use milk or soymilk in your coffee? Is the milk organic? Are the pastries made in house or shipped from a warehouse? Are they sweetened with high fructose corn syrup or honey? And on and on. These represent only a fraction of the choices that are made by urban individuals each day ${ }^{2}$.

This thesis is concerned with identifying and exploring the values that inform food choice for the urban consumer in North America. What do individuals look for when they make these choices? What matters most? What makes food 'good'? I argue that making choices about food, from this dizzying array of food options, constitutes one of the most significant components of modern urban life. Consumers are constantly

\footnotetext{
${ }^{2}$ It is important to stress that my analysis explores the perspective of a very small, elite group of people who have the time, economic/physical resources and ability to make the types of food choices I describe. The majority of people do not have this ability due to their social class, geographical, racial or political position in the world. I do not present this analysis as a global perspective (i.e. everybody has these choices to make and everybody does make these choices), but rather the perspective of a small population of privileged North Americans. I recognize, and find it prudent to highlight, this bias in my research population and in my discussion of this material.
} 
reminded that food choices matter, both on a bio-chemical level, affecting the body in capacities of health and aesthetics, and as a potent signifier of economic status, ethical stance, and cultural affiliation (Long, 2004). Through these food choices, individuals construct and present who they are.

My research explores this link between food choice and identity by studying consumer practices at a popular restaurant in Ottawa's downtown core: The Green Door Restaurant. I use this restaurant as the primary focus of my study because it serves as a clearly definable site of urban food consumption. In this study, I explore how food choices are enacted within this distinctly constructed social space, and investigate how these food practices are experienced and perceived by their patrons. In doing so, I have been able to identify core values that inform food choice within this space and explore how these values are implicated in processes of self.

Specifically, I have engaged in several months of ethnographic field observation at The Green Door and conducted 15 in-depth semi-structured interviews with 15 different patrons. I investigate three primary research questions:

- What are the food values being enacted in this space?

- What are the implications of dining in this social space for the individual in terms of presenting a 'valued' identity?

- What does the prevalence of particular food values reveal about the state and direction of our contemporary urban foodscape?

The observations I made and the responses I received over the course of my research have affirmed my contention that food choices are meaningfully implicated in processes 
of identity for the urban individual. I have found that making choices about food is indeed regarded as an important challenge of urban life, and that urban individuals devote a great deal of time and energy to making 'good' choices. In this thesis, I explore one food value that emerged from my data as a significant, and shared, qualifier of 'good' food: the value of 'fresh.'

In this thesis, I look at how 'fresh' is enlisted by my research participants as a core value of contemporary urban food consumption. My data indicates that urban individuals do not simply revel in the abundance of choice and variety that is available in our industrialized food system; rather, they engage in processes of reflexive decisionmaking in efforts to construct a personal food practice that aligns with their ethical concerns. I suggest that 'fresh' has emerged as a core food value within this reflexive construction of personal food practices because it appeals to consumer appetites and addresses concerns in a way that other food qualities do not. A 'fresh food ethic' permits flexibility as these individuals navigate between their food values and construct a personal food practice that is 'good.'

There are several important sociological implications of this dynamic. Focusing first on patrons' comments about food choice in their everyday life, I show how the way 'fresh' is valued by my participants exemplifies the increasing responsibilization of the individual in the modern neo-liberal era. I see that each of these individuals is tasked with being an informed, responsible consumer and making the 'right' choices to ensure optimal standards of health and wellbeing. This process of choosing correctly is a 
significant dynamic in terms of how food choices are implicated in the presentation of self. It also has important implications in terms of how individuals participate as consumers in a supposedly 'democratic' industrial food system.

In a system that urges consumers to 'vote with their wallets' to make 'good' choices, 'fresh' is implicated in the marketing and sale of food; it assures consumers that this food is 'good' for them and will support them in their efforts to present a responsible, 'ethical' self. I demonstrate that The Green Door constructs a 'fresh' space for its patrons in order to create a dining experience that will be attractive to urban consumers. Looking specifically at how 'fresh' operates as a core value within The Green Door, I show that 'fresh' functions within an existing framework in the sociology of taste; contemporary food values are presented in seemingly democratic terms while functioning covertly as signifiers of status and distinction (Johnston \& Baumann, 2010). My findings exemplify this research and, like Johnston and Baumann's work, highlight the limitations of marketbased, consumer-choice solutions to the environmental and social problems that afflict our contemporary Canadian food system.

The primary contribution of this thesis emerges out of this analysis. By focusing on how food choices are linked to processes of self, I identify a dynamic that is not acknowledged in Johnston and Baumann's work on culture and taste. Whereas Johnston and Baumann (2010) argue that environmental and class issues are trivialized in contemporary food discourse, I demonstrate that individuals do engage in reflexive thinking about their food choices and recognize that their ability to act on 'fresh' values 
reflects a position of privilege. My data indicates that individuals do not blindly buy into market promises of status and distinction; rather, they acknowledge the fundamentally unethical nature of the system they engage in and engage with 'fresh' values in genuine efforts to make responsible, 'ethical' choices. This added complexity is significant because it results in a critical breakdown in the presentation of self for the urban consumer.

Despite the considerable efforts of educated, affluent individuals to make responsible food choices, these practices can never actually satisfy the ultimate goal of presenting a responsible, 'ethical' self. In an industrialized urban food system that relies on class inequalities and externalized environmental costs, the failure of the individual to present a sufficiently 'responsible,' 'ethical' self is inevitable. Importantly, the responsibilization of the individual as consumer-citizen not only has damaging consequences on the individual; it also ensures that the systemic problems of our industrialized food system are not addressed. I argue that as long as individuals are preoccupied with the minute successes and inevitable failures of their personal project-ofthe-self, they cannot be engaged meaningfully in collective action. I propose that it is necessary to re-think this individualist consumer model if we indeed hope to create a food system that is 'fresh.' This analysis exemplifies a significant dynamic of contemporary food choice and serves as an essential contribution to the growing body of food studies literature. 


\section{Thesis Outline}

I will begin by describing the basic premise of my research. I explain my rationale for exploring food choice within the context of the restaurant and introduce The Green Door Restaurant as the focus of my study; I will briefly describe its mandate and presence in Ottawa and outline the value of this restaurant as a site of sociological investigation. I then outline the broad theoretical framework that has informed and supports the project design. In Chapter 2, I provide a brief review of the sociological literature that has informed my research, including existing work on regulating the self, food and choice, the industrialization of food, sociology of taste, and 'freshness.' In Chapter 3, I offer a detailed summary of my methodology. Chapters 4 and 5 constitute the bulk of the analysis. In Chapter 4, I outline the five core food values that emerged from my research and argue that they serve as component parts of a larger 'fresh food ethic.' I propose that freshness acts as an overarching signifier of 'good' food, appealing to consumer desires and quelling fears in broad strokes that resolve inconsistencies and hypocrisies. I argue that the 'fresh food ethic' has emerged as a viable mediator and consolidator of food values within the reflexive construction of individuals' personal food practices. In Chapter 5, I explore how 'fresh' is engaged and enacted within The Green Door Restaurant to offer consumers a desirable dining experience. I propose that 'freshness' exemplifies an ideological tension between democracy and distinction that characterizes contemporary gourmet discourse. However, I qualify this analysis; whereas this dynamic generally indicates a dismissal or trivialization of class inequities, my interviewees 
demonstrate a self-consciousness around privilege that further complicates their relationship with food choice. In the sixth and final chapter, I apply my findings to a broader social context. I address larger questions about the responsibilization of the individual as consumer and the potentialities of consumer-based activism. I explore the sociological significance of 'fresh' in terms of how it has become implicated in processes of reflexive decision-making, and how it defines, shapes, supports, and challenges the larger mechanisms of our contemporary urban foodscape.

\section{Food Choices in a Distinct Site of Urban Food Consumption}

In order to explore the values that inform food choice in a contemporary urban food system, I have focused my attention on one specific domain of urban food choice: the restaurant. 'Dining out' constitutes an increasingly widespread practice for urban individuals. Once considered a luxury restricted to elites, the social event of going to a restaurant has been democratized to the extent that dining out is now extremely common across a wide range of social demographics: "most people eat out sometimes, many do very often" (Martens \& Warde, 1995: 131). I argue that restaurants are a particularly appropriate focus for research on urban food choice because they highlight the shifting scope and dynamics of food choice in urban centers.

In any urban center, restaurants dedicated to every conceivable ethnic, dietary, and stylistic cuisine offer consumers unprecedented access to food choice. In this capacity, the restaurant exemplifies the expansion of food choice for the urban individual. Because restaurants are in the business of appealing to consumer desires, I suggest that 
selecting a successful, popular restaurant as a site of study can offer relevant insight as to the contemporary values that attract clientele. The restaurant also serves as a good site of research on food choice because it offers extraordinary opportunity for individual choice within each dining experience.

The restaurant meal is distinct from the traditional domestic meal (where one meal is prepared and shared by a group) in that it is selected individually, usually with options to customize the plate. In this way, restaurants reflect the individualistic attitude that now characterizes urban food choice. The restaurant also alters the dynamics of domestic food consumption by bringing the traditionally private act of dining into the public sphere. In the context of a restaurant, an individual's choices are made in a distinctly social context, "during a period marked by his continuous presence before a particular set of observers" (Goffman, 1959: 22). I argue that this highlights the symbolic value of food choices and propose that the choices enacted by individuals in this context are importantly implicated in a performance of self (Goffman, 1959).

The restaurant constitutes a particularly good site of study for my thesis because it grounds my analysis in the concrete action of consumer choice. I see that restaurants construct a distinct social context that reflects and informs patrons' food values. In my research at The Green Door, I made note of the physical constructs of the space, including spatial design, lighting, furniture, and artwork/décor, as well as the processes and practices that are enacted there. I watched individuals interact with the space, staff, and one another as they navigated through this space, and directly observed patrons 
select, pay for, and consume their food. In doing so, I gained important insights about how food consumption is 'framed' within this space, who goes to this restaurant, and how food choices are 'lived out' in action.

Because this thesis is concerned with exploring the link between food choice and processes of identity, I was particularly interested in how food practices in this space are perceived by their patrons; it is these perceptions that are meaningfully implicated in their individual processes of self. Selecting a restaurant as a primary site of study, and then recruiting patrons of that restaurant as interviewees, has proven to be a strong research model for this project because it provides a common reference point for interview participants - i.e. the restaurant - in an otherwise extremely broad topic of discussion, and focuses the interaction on food practices, as opposed to ideological musings about food choice.

Focusing my research on urban food choice at a restaurant has allowed me to investigate the values that individuals bring to their consumption practices in this space, and explore how these choices are manifest in action. I will now introduce the specific restaurant I have selected as my primary site of study, and offer a brief explanation as to why this particular restaurant serves as a strong choice.

\section{Entering Through The Green Door}

Since 1988 , the daily practice in the kitchen and bakery of the Green Door Restaurant reflects our desire to create delicious and wholesome food that is as close to its natural state as possible. We do this by keeping things simple; there are no fillers, processed ingredients, or preservatives in any of our dishes. We work from 6:00 am until 10:30 pm kneading bread, creating desserts, stirring soups, preparing vegetables, cooking, chopping, slicing, blanching, sprouting, 
blending, baking, roasting, serving, juicing, brewing, wiping, washing, dishing, scrubbing and placing food on the buffet. Thirty people on our team repeat the same process, behind the scenes and in front at the cash, 6 days a week.

(Guide To The Green Door, 3)

The Green Door, "Ottawa's oldest and most loved vegetarian restaurant," has established itself as a thriving dining destination (Guide to the Green Door, 2010). Described in the passage above, this restaurant is constructed around a menu of locally sourced, 'simple' food, which is served buffet-style and priced according to weight. The food is presented as 'healthy' and 'wholesome,' and is exhibited in colourful, abundant, glistening displays in front of the open-concept kitchen where it was prepared. Customers, themselves, construct their meals, lining up to choose the specific dishes and quantities they want from a daily spread of soup, breads, curries, stir fries, lasagna, cold salads, and desserts. Each of these dishes, totaling upwards of 50 each day, are labeled with friendly, handwritten cards that name the dish and give information about its 'key ingredients.' Once patrons have had their plates weighed and priced at the cash, those who choose to dine-in carry their trays to one of the many tables arranged densely in an open-concept cafeteriastyle dining space. Patrons can also choose to fill take-away containers if they wish to take their food to go.

I selected The Green Door Restaurant as the focus of this study because it represents a distinct approach to food choice that clearly appeals to consumer desires, while also inviting interpretative analyses from its patrons. When I first moved to Ottawa, I was told to go to The Green Door by nearly everyone I spoke to about my interest in food. All of these people offered personal interpretations of the restaurant. The Green 
Door was described as one-of-a-kind; a local gem that had been around for many years, was always busy, and served amazing food. I was given the distinct and sustained impression that The Green Door Restaurant is a provider of the things that are 'good' about food; patrons celebrated the quality of the food, while also alluding to the environmental and social merits of the restaurant's operations. Both the food and the atmosphere also seemed to provoke critical thinking about the social dynamics of the dining practices (including the incongruence between The Green Door's socially conscious mandate and its high prices) and inspire thoughtfully formulated interpretations about urban food practices (such as what it means to eat 'organic'). The people who advised me to go the restaurant shared these musings and, in doing so, inspired my sociological investigation.

When I had my first 'Green Door experience' it became immediately apparent most notably through the line-up that wove around the buffet line and out the door - that this restaurant enacts a set of values that are highly desired by its patrons. I provide a more detailed rationale for my selection of the restaurant in Chapter 3, but at present let it suffice to say that focusing my research on The Green Door Restaurant has allowed me to both identify some key urban food values and investigate the implications of these values in terms of how they are played out in a defined social space.

\section{Theoretical Framework}

In terms of an epistemological approach, my design relies broadly on a social constructionist perspective. Some constructionists believe that "there is no such thing as 
a single objective reality" but rather multiple realities that develop through the discursive processes of individual and collective interaction (Feilzer, 2010:6). I recognize that a restaurant, like any social space, is a construct derived from, and dictated by, the "views, actions, and interactions" of individuals and groups "at a particular time and place" (Charmaz, 2006: 189). More precisely, my research project is grounded in a symbolic interactionist perspective, which emphasizes that "people act towards things on the basis of the meanings they ascribe to those things" (Blumer, 1969). Arguing that food is a particularly potent social object (Lupton, 1996), this perspective allows me to investigate how the restaurant serves as a symbol-laden site of social interaction.

I have also drawn on Goffman's (1959) The Presentation of Self. Goffman proposes a dramaturgical approach to social life wherein the process of self-production is understood as a performance. He employs a theatrical metaphor that includes the back and front stage, actor, performance team, and audience to create a framework for understanding the inner-workings of social life. In Goffman's model, the actor creates impressions for his/her audience through sign-vehicles; expressions of appearance and manner that culminate in what ultimately become the performance of self. The notion of food as a stage prop in the performance of self captures both the literal and symbolic qualities of food and highlights its role as an essential component of social life. Goffman emphasizes that his dramaturgical perspective is particularly applicable within concrete social establishments where "social life is organized within the physical confines of a 
building or plant" (Goffman, 1959: xi). The Green Door Restaurant is a closed social establishment where food is employed as a primary prop for the performance of self.

Interestingly, Goffman uses an example of a waiter, moving awkwardly, then playfully, then mechanically throughout a restaurant, to illustrate how actors play with appearance and manner in order to realize their condition (75). It is this sense of play, this exploring the boundaries of one's existence, which I find to be particularly applicable to processes of food choice in modern society. Because of the abundance of options available to the modern urban consumer, the individual can engage with food in highly specific ways that signal who they are (or who they want to be).

While there is a widely acknowledged emphasis on the individual in modern Western culture, Goffman stresses that teams, rather than individuals, are his basic unit of analysis for studying the social world. A performance is not a solo endeavor, but rather requires the collaboration of multiple actors who work closely with one another to pull off the show. I entered my research with the understanding that there is a huge amount of cooperation that goes into operating a restaurant and staging a dining out routine.

Goffman's model has encouraged me to look not only at individual narratives about their food values, but also at the collaborative processes and practices that shape individuals' experiences of, and perceptions towards, food consumption. Restaurants, which rely on mechanized processes of food production and distribution to cater to a mass clientele, and often employ culinary techniques and ingredients from across the globe, exemplify shifting dynamics in these collaborative processes that have resulted from the 
industrialization and internationalization of our modern food system. I argue that Goffman's work is particularly useful in my investigation of urban food consumption because of his attention to these important (and shifting) social dynamics.

While Goffman's theatrical metaphor has very much informed my research, I have not chosen to explore the nuances of conceptualizing food as a prop or patron as a performer as the primary focus of my analysis. I have begun to develop these ideas in other work (and indeed see this as an immensely interesting avenue to pursue), but, recognizing the limited scope of the Master's thesis, I have chosen not to detail this analysis here. Rather, I introduce this framework as a foundational theoretical model for my work. Goffman's work has served as the overarching metaphor through which I have been able to explore how 'dining out' is implicated in the modern project of the self.

In the next chapter, I will review the existing literature that has informed my analysis of 'fresh' as a core value in contemporary urban food consumption. I provide an overview of the work that is particularly relevant to each layer of my analysis and illustrate how my thesis fits into this existing body of academic work. 


\section{CHAPTER 2}

\section{LITERATURE REVIEW}

This thesis explores the values that inform consumer choice in our contemporary urban food system. I identify 'fresh' as a core food value, and explain how it functions as a qualifier of 'good' food for urban consumers, to argue that individuals' engagement with 'fresh' highlights consumer efforts to present a responsible, 'ethical' self. This has important implications both for the individual, who is caught in an unending process of reflexive decision-making, and for the larger urban food system, which relies on unsustainable, socially problematic practices that cannot be rectified through individual counter-consumption. Our collective failure to challenge industrialized food production and distribution practices - such as the heavy use of pesticides and antibiotics, the precarious employment of low waged migrant workers, and the aggressive marketing of highly processed convenience foods - is significant because it disrupts individual efforts to present a valued self, and has devastating environmental and social consequences including, but not limited to, soil depletion, climate change, inhumane working conditions for farm workers, and increasing rates of heart disease, diabetes, and obesity. My study explores these dynamics of contemporary Western food choice.

The purpose of this chapter is to situate this analysis in an existing body of food studies literature. I provide an overview of the work that has been particularly influential to my analysis and illustrate how this thesis fits into this existing body of academic work. Many scholars have contributed to our current understandings of contemporary cultural 
meanings around food; this literature review offers only a snap shot of the exciting work that now addresses the many trends that shape modern society, and our current food system in particular, and inform (and complicate) food choice.

My research has emerged out of an increasingly noted understanding that the industrialization of our contemporary Western food system has fundamentally changed the food consumption practices of urban individuals (Mennell, 1995; Haden, 2005; Winson, 2005). Many scholars acknowledge that food choice is complicated in an industrialized food system that both provides incredible possibility for culinary exploration and confronts consumers with a myriad of anxieties and dangers (Lupton \& Chapman, 1995; Guthman, 2003; Johnston et al., 2009). I begin by providing an overview of this literature, which serves as the foundation of my research. The dynamics of food choice I summarize in this section are critical to my analysis; I argue that 'fresh' serves as a catch-all qualifier of 'good' food, easing the anxieties of managing food choice by appealing to consumer desires and addressing their concerns. This analysis is significant because individuals' engagement with a 'fresh food ethic' highlights their efforts to present a responsible, 'ethical' self.

The analysis that has emerged from my research on food choice fits into a substantial body of literature on the responsibilization of self in modern society (Lupton, 1996; Giddens, 1991; Foucault, 1978). To contextualize this component of my analysis, I will summarize the work of a number of sociologists who argue that the modern individual is framed primarily as an autonomous, responsible risk-manager. I then point 
to sociological literature that explores how particular food values are employed in the commodification of food products in such a way that reflects this responsibilization of self.

Next, I focus specifically on a recently published book entitled, Foodies: Democracy \& Distinction, which provides the sociological framework I employ in the second piece of my analysis (Chapter 5). In this book, Johnston and Baumann (2010) propose that food values function within competing ideologies of democracy and distinction in contemporary gourmet discourse. This work is essential to my thesis because, as I demonstrate in my data analysis, 'fresh' exemplifies the tension between these competing ideologies and points to a dynamic within this framework that has not yet been explored. I build on Johnston and Baumann's analysis by showing that consumers recognize that their engagement with 'fresh' reflects status and privilege; I argue that this recognition complicates, and ultimately sabotages, their efforts to present a responsible, 'ethical' self. This type of recognition-without-meaningful-action matters both for the individual, and for the larger food system, which continues to rely on unsustainable, inequitable practices unchecked.

Last, I will look at the fascinating (but notably limited) work that has been produced to date on the function of 'fresh' in a contemporary Western foodscape. This summary of previous writings provides relevant background information about the food value that is at the centre of my analysis. 


\section{Making Food Choices}

Changing cuisine and food practices in our contemporary Western food system has been noted and explored in much sociological literature (Mennell, 1985; Haden, 2005; Winson, 2005). These scholars describe the industrialization, commercialization, and internationalization of food production and distribution in modern urban centers to trace how rapid 'advances' in technologies of the food production and distribution industry have come to offer consumers "the opportunity for more varied experience in eating and to develop more varied tastes" (Mennell, 1987: 321). Technologies of controlled ripening, refrigerated transportation vehicles, protective sprays, and protective packaging (to name only a few), have reduced differences in availability based on season or social occasion (Mennell, 1995), and enabled the mass production of highly processed, "enhanced," convenience food products, which now saturate our food system and shape contemporary food practices (Haden, 2005). These shifts in the contemporary Western food system have created unparalleled choice for consumers.

Many food scholars (Guthman, 2003; Bordo, 1993; Ashley et al., 1995; Haden, 2005) acknowledge that our contemporary Western food system has evolved in such a way that making choices about food constitutes a significant social challenge. The variety and scope of food choices available to the average urban consumer requires that they make detailed choices about food multiple times a day. Lupton and Chapman (1995) write that individuals are "subjected to imperatives" right from infancy that indicate the kinds and quantities of food that they should eat to maintain good health and 
physical wellbeing (477). They argue that adults continue managing food habits and regimens through internal regulation, "the practices of the self that have been normalized through acculturation," and external regulation, "via the imperatives of health promotion and medical advice" (478). This task of managing food choice is difficult because, as many food scholars (Fischler, 1990; Lupton \& Chapman, 1995; Guthman, 2003) have observed, the contemporary cultural meanings that inform these choices are rife with paradoxes, including those related to competing social and physical needs, and cost. Food operates in "dual and conflicting roles" of possible pathogen, a source of disease and risk, and life energy, source of nourishment and pleasure (478).

Claude Fischlers' (1990) seminal work L'Homnivore provides a useful foundation for conceptualizing food choice. Fischler identifies the 'omnivore's paradox' as a formative dynamic of human relationships with food. He observed that humans have a biological need for variety, diversity, and innovation in food that competes with an equal, and opposing, need for caution against unknown foods that may be dangerous. $\mathrm{He}$ suggests that societies develop rules, norms and classification systems around food consumption and preparation in order to resolve the tensions and anxieties that result from these competing needs (1988: 278-279).

Other scholars illustrate that our contemporary urban food system is indeed characterized by distinct classifications of 'good' and 'bad' food (Lupton \& Chapman, 1995). For example, Lupton and Chapman (1995) note that 'good' foods are healthy and non-fattening, while foods that are unhealthy (read: fatty or sugary) are deemed 'bad.' 
They note that many of the health risks associated with a 'bad' diet are made clear through widespread media coverage and government messaging that warns against the risks of a 'bad' diet, including obesity, diabetes, and heart disease (Lupton \& Chapman, 1995). Choosing these 'good' healthy foods, however, can be more complicated than simply following government guidelines. Haden (2005) shows that many highly processed edible products are advertised to highlight the supposed 'goodness' of the food with promises like 'vitamin-enriched,' or 'low-fat,' which complicate consumer efforts to eat nutritious, 'good' food. Additionally, food is not only valued from a 'nutritional science' perspective that prioritizes biomechanical function (Lupton, 1996: 92), but is also charged with cultural meanings related to group affiliation, cultural tradition, and aesthetic ideals.

Lupton and Chapman (1995) point out that categories of 'good' and 'bad' food are further complicated by social factors. Foods are valued or avoided based on social occasion and cultural membership. Additional paradoxes emerge out of these social meanings; for example, many people experience a tension between the need to eat certain types of 'good' food for health or aesthetic reasons, and the need to eat 'bad' foods for comfort, pleasure, and belonging (478). One important paradox exists in the financial limitations of particular social classes, which prevent individuals from making 'good' choices. Choices of necessity may be complicated by social meanings that set up the consumer as 'bad' and 'unhealthy.' On the other hand, a number of scholars note that contemporary food choices among the upper classes are affected by increasing pressure 
to achieve and maintain an arguably impossible standard of slimness (Guthman, 2003; Bordo, 1993). Guthman argues that "yuppie eaters" who can afford to pay top dollar for a plate of organic mixed greens pride themselves on restrictive eating on the grounds that it reflects "conscious reflexivity" and "moral valence" (Guthman, 2003: 45). Noting the tension between an individual's physical hunger and their efforts to maintain a socially approved body, these scholars demonstrate that negotiating various, and often contradictory, food 'needs' constitutes a significant challenge for modern individuals.

Scholars note that people develop strategies to negotiate between these meanings of food choice (Ashley et al., 2005; Guthman, 2003; Bordo, 1993). Ashley et al. (2005) suggest that individuals engage in a process of 'calculated hedonism,' wherein the restraint and discipline associated with the ideal diet is balanced carefully with calculated pleasures of self-indulgence (202). Guthman (2003) notes that the stakes are high for individuals attempting to negotiate this balance, as those who make too many 'bad' choices are not only sentenced to suffering negative health effects, but are also de-valued as moral failures. In a food culture that simultaneously promotes indulgence and discipline, eating becomes "an exercise in the management of anxiety" (Ashley et al. 188).

My analysis is grounded in, and contributes to, this body of work. I explore what makes food 'good' and identify 'fresh' as a core value of food choice; I then investigate the implications of this food value in terms of how the urban individual negotiates food paradox. In accordance with the scholars reviewed above, I see that distinctions between 
'good' and 'bad' food have important, and varied, ramifications. Based on my data findings, I argue that 'fresh' eases the anxieties of managing food choice by appealing to consumer desires and addressing concerns in broad strokes that resolve the paradoxes and hypocrisies of extensive urban food choice.

\section{Making 'Responsible' Choices: Looking at the Logic of The Regulating Self}

The analysis that has emerged from my research on food choice fits into a substantial body of literature on the responsibilization of self in modern society (Lupton, 1996; Giddens, 1991; Foucault, 1978). In an era of modernity and neo-liberal politics, there is a great deal of emphasis on individual responsibility and self-governance (Fusco, 2006). Numerous scholars have shown that contemporary Western society operates within a neo-liberal ideology that seeks to minimize involvement on the part of the state, and emphasizes self-responsibility, self-surveillance and individual autonomy; the individual is viewed as a moral agent who voluntarily takes up self-governmental imperatives (Lupton, 1999; Lupton \& Chapman, 1995; Giddens, 1991; Foucault, 1978).

In contemporary Western societies, food tastes and habits are highly related to understandings of the 'civilized self' (Elias, 2000); "the self who understands and accepts that certain foods are more appropriate than others to eat" (Lupton \& Chapman, 1995: 478). Certain foods are deemed to be appropriate, 'good' and 'civilized' foods, while other foods are 'bad' and betray self-indulgence, and a lack of taste and social refinement (478). Lupton has produced a number of works (Lupton, 1999; Lupton, 1996; Lupton \& Chapman, 1995), which illustrate the link between the internal governance of the 
individual and choices related to health. She shows that governing the self through health-related choices, particularly those related to diet and exercise, are deeply influenced by perceptions of risk. Food is a particularly significant social object because it is susceptible to contamination and, being ingested, is risky at the most intimate level.

Specifically, Lupton's work draws on Gidden's understanding of the 'risk society,' which conceptualizes modern existence as "living with a calculative attitude to the open possibilities of action, positive and negative, with which, as individuals and globally, we are confronted with continually" (Giddens, 1991: 28). Lupton (1995) suggests that within this 'risk society' the discourses of a new public health would have us believe that health risks "loom around every corner, posing a threat to the public" (77). The individual is not only confronted with micro-level concerns (such as hygiene and sanitation), but also the risks of macro environments and pollutants, including concerns about radiation, climatic change, and industrial waste. These risks, which produce largescale threats to the human species, require the vigilance of the 'environmental citizen' who is focused on making responsible, safe choices to maintain personal health and minimize the ecological damage of industrialization (Petersen \& Lupton, 1996).

Importantly, Lupton indicates that people do not only transform themselves into subjects of self-regulation as a means of avoiding potential threats, but also in the interest of self-improvement. The project of the self is not only about mitigating risk, but also achieving some ideal of happiness and healthiness. As issues around nutrition, fitness, pesticide use, animal cruelty, and labour rights take center stage in popular public 
discourse, notions of 'ethical' consumer choices are interwoven with notions of self-care. Lupton (1999) states:

Risk-avoidance behaviour, therefore, becomes viewed as a moral enterprise relating to issues of self-control, self-knowledge and self-improvement. It is a form of self-government, involving the acceptance and internalization of the objects of institutional government. Because the project of selfhood is never complete, but rather is continuing throughout the lifespan, so too the project of risk avoidance as a technology of the self is never ending, requiring eternal vigilance (p. 91).

I find Foucault's (1978) concept of governmentality particularly useful for conceptualizing the projects of self that appear in my data. Foucault indicates that individuals are governed not only through a top-down imposition of power, but also through mechanisms of internal self-government. He (1978) argues that we now experience a new 'administration of life,' whereby methods of training, self-surveillance, normalization and examinations constitute an 'anatomo-politics' of the human body that allows for more effective governance of society. Individuals' efforts to make 'good' choices are regulated primarily, if not solely, via mechanisms of self-government. For example, an individual may not cite any one authority who monitors their food consumption, or a single expert whose guidelines they follow; rather, they might draw on multiple bodies of food knowledge to construct highly individualized personal food ethics and rely on self-surveillance to maintain a personal food practice that is 'good' for them. The concept of governmentality brings together Foucault's interest in "the micropolitics of the body, the macro-surveillance of population, and the technologies of selfsurveillance, which together provide for a comprehensive management of life" (Fusco, 
2006). Discourses of risk encourage individuals to police themselves to avoid risk and achieve cultural ideals through self-surveillance and self-improvement (Lupton, 1995).

These scholars' conceptualizations of how a modern, neo-liberal ideology links to individual governance and health are fundamental to understanding the logic of food choices. They establish the individual as an autonomous, responsible self, and provide the base from which I explore how food values are implicated in efforts of risk management and self-improvement. As will be seen in the data analysis chapters of this thesis, my interviewees demonstrated a 'calculative attitude' towards their food choices in their efforts to act 'ethically' in terms of their personal health and the health of the planet. My data indicate that individuals' engagement with 'fresh' exemplifies the larger social implications of a micro-politics of the body; developing and adhering to a highly personalized 'fresh food ethic,' the individual engages in continual, and inevitably futile, attempts to present a responsible, 'ethical' self, while leaving the fundamentally unsustainable and inequitable practices of our contemporary Canadian food system unchecked and unchallenged.

\section{Marketing Food Values}

It is also relevant to this thesis to consider how food values such as 'fresh' are implicated in corporate efforts to capitalize on the types of ethical (i.e. environmental, social, and nutritional) concerns that preoccupy the responsibilized urban consumer. Freidberg (2009) asserts that a culture of industrial capitalism demands that we consume "as often as possible" while navigating the "anxieties and dilemmas of mass consumption" by 
shopping "in ever better, more enlightened ways" (3). This assertion points to the core 'problem' addressed in this thesis; I explore how individuals engage with notions of 'fresh' as a means of presenting a more 'enlightened' or responsible self while navigating the personal anxieties produced by the demands of a culture of mass consumption. Many studies explore the various ways companies construct food products, or more accurately, brand identities, that appeal to a consumer's ethical concerns, fitness goals, personality traits, dietary restrictions, and cultural affiliations (Parkins \& Craig, 2009; Roseberry, 1996; Wilson, 2004). In the context of a contemporary urban food system, food marketing plays a powerful role in 'helping' consumers decipher the value of food products and make 'smart,' 'good' selections.

Lupton (1995) points to the extent to which environmental and health risks have been taken up as subjects of widespread, popular consumption; she notes that "risk assessment and risk communication have become growth industries" (77). That is, the business of 'helping' consumers mitigate risk - which operates primarily (if not solely) to maximize profit - has taken up an important, and increasing, presence in consumer culture. This is significant in terms of what kinds of information and messaging are available (and promoted) to consumers as they make choices as 'environmental citizens' about food.

Johnston et al. (2009) point to another relevant dynamic in food marketing; they demonstrate that 'alternative' values are easily commandeered by large corporate players. These researchers investigated what they term the "corporate-organic foodscape," 
identifying stock themes (including reference to specific geographic location, small-scale, pastoral food production, humble origins, and commitment to family) that are employed as strategies to humanize the "behemoth transnational corporations" and garner the trust of the consumer through a manufactured "personal relationship" (512). They found that companies engage with the language of alternative movements to appeal to consumer desires, while continuing to rely on industrial mechanisms of food production and distribution to maximize profit and cater to mass demand. Johnston et al. argue that 'alternative' terms like 'organic' have been "fetishized;" they are employed as marketing tools to appeal to emotional sentiments rather than reflect sustainable farming practices (Johnston et al., 2009: 511).

My study contributes to this body of literature in that I explore how 'fresh' is taken up in the commodification of food at The Green Door Restaurant. In accordance with this literature, I argue that individuals' engagement with 'fresh' in this space reflects their efforts to consume in ever more 'enlightened' ways to mitigate risk and make 'good' choices. Further, and similar to Johnston et al., I see that efforts to present a responsible, 'ethical' self are complicated by a disconnect between widespread conceptualizations of alternative food values (like 'fresh') and the real practices of the industrialized food system. In the next section, I look at how food values - like 'fresh' operate within our industrialized, commodified food system as signifiers of 'good' taste. 


\section{Sociology of Taste}

Johnston and Baumann's (2010) Foodies: Democracy Vs. Distinction in the Gourmet Foodscape has been particularly relevant to my research because it provides a framework for understanding how food values function in our contemporary Western food system. Through an analysis of texts from food magazines and blogs, as well as interviews with self-proclaimed 'foodies,' Johnston and Baumann document and explore how new markers of high-status food have emerged in North America. They argue that contemporary gourmet food discourse overtly reflects a liberal democratic ideology of equal opportunity and cultural openness, while functioning covertly to promote an ideology of status and distinction (Johnston \& Baumann, 2010: 42). In a cultural climate where gaudy displays of wealth and overt snobbery are no longer accepted, individuals distinguish themselves as high status in new ways. Specifically, Johnston and Baumann see that notions of authenticity and exoticism, both articulated as egalitarian ideals, "work to signify specific foods as a source of distinction for those with cultural and economic capital" (35). Through a systematic discussion of this trend, they conclude that by valorizing these seemingly democratic values, contemporary food discourse minimizes and obscures fundamental class inequalities.

These authors provide an immensely rich and comprehensive review of the sociological literature on food, culture, and taste. In this section, I outline a number of key ideas that form the foundation of their analysis and inform the examination of my own data. The notion of "omnivorousness," used increasingly by sociologists of culture 
in the last several decades (Warde et al., 2007; van Eijck, 2001, Fischler, 1990), serves as a foundational concept in Johnston and Baumann's work. These researchers claim that omnivorousness, defined literally as openness to eating various plants and animals, serves as a useful concept in understanding the complexity of contemporary cultural (including culinary) trends. Johnston and Baumann illustrate that people with high levels of education and income tend to have cultural tastes that sample selectively from both traditionally highbrow and traditionally lowbrow genres as a means of signifying high status. Omnivores are not less status seeking, but rather are granted high status according to alternative values.

To explore this concept of omnivorousness, Johnston and Baumann look to Bourdieu's work on taste and distinction. Contemporary mass consumption has resulted in a decline in the ostentation and extravagance of haute cuisine and a 'democratization' of eating (Mennell, 1987). Johnston and Baumann argue that despite these shifts, gourmet culture can serve to affirm and elaborate Bourdieu's key assertion that everyday cultural forms - like food - are instrumental in creating and maintaining social status and distinction (34). Bourdieu's Distinction (1984) illustrates that the "consecration of certain cultural preferences as part of 'good taste' is not a random phenomenon, but reflects the ability of dominant class fractions to legitimate their tastes as superior" (Johnston \& Baumann, 2010: 33). Johnston and Baumann suggest that an omnivore's valuation of food indeed reflects this paradigm, highlighting status for an elite and maintaining social hierarchies. Acknowledging that contemporary taste hierarchies are 
no longer simple or straightforward, they argue that there remains to be "an important relationship between social class, power, and aesthetic preferences as they relate to food" and that "the everyday life concerns of eating can say a great deal about the relationship between taste, class, and power" (34).

Johnston and Baumann examine how notions of authenticity and exoticism, which they identify as key elements of foodie discourse, function as the primary goals and motivations of the contemporary gourmet. While Johnston and Baumann provide an immensely detailed discussion of these themes, I provide only a brief summary here. First, Johnston and Baumann explore how references to authenticity are implicated in narratives about food. Specifically, they show that food is "understood as authentic when it has geographic specificity, is "simple," has a personal connection, can be linked to a historical tradition, or has "ethnic" connections" (70). Johnston and Baumann argue that an emphasis on authentic culture appears to facilitate a democratic ideology - valuing the food cultures of marginalized groups, and helping to sustain small-scale and local food producers - while simultaneously drawing clear, discriminating lines between legitimate and illegitimate foods.

Exoticism is the other key value in contemporary gourmet discourse identified by Johnston and Baumann. Situating their discussion in Said's (1978) seminal work, Orientalism, which explores Western culture's fascination with the exotic Other, these authors observe that foodies place high value on foods from different countries and nonEuropean ethnicities (Johnston \& Baumann, 2010: 111). They note that 'culinary 
cosmopolitanism' - a willingness to eat the food of others - may reflect a growing democracy of the palate and an admirable appreciation for culinary diversity, but must also be critiqued as an imperialist enterprise. Finding new exotic cuisines earns 'foodies' social status based on currencies of food knowledge and adventurous norm-breaking. Johnston and Baumann demonstrate that like 'eating authenticity,' 'eating the Other' is regarded as an important strategy for validating the eater as high status in an omnivorous culinary discourse.

Johnston and Baumann's work on how food is valued in gourmet discourse is relevant to my research in that it has offered a framework for understanding how food values function as markers of status and distinction in our contemporary Western food system. This thesis serves as an extension of Johnston and Baumann's analysis by looking specifically at how a contemporary urban food value, 'fresh,' is lived out in the act of dining. Given my data findings, I argue that like authenticity and exoticism, 'fresh' operates within a paradigm of omnivorous culinary consumption; educated, relatively well-off patrons of The Green Door view the markedly casual, cafeteria-style dining experience as an attractive and desirable food consumption practice. I conclude that 'fresh' serves as an overtly democratic ideal, while functioning covertly as a marker of distinction.

My analysis also builds on Johnston and Baumann's work by suggesting that, whereas 'fresh' is unarguably implicated in signifying distinction and obscuring class inequalities, it is also meaningfully implicated in processes of identity management that 
cannot be reduced solely to status display. My research indicates that 'fresh' is implicated in internalized practices of self-surveillance and self-improvement, which are strictly managed in a reflexive project of the self that relies on an 'omnivorous' engagement with multiple and varying bodies of food knowledge. I illustrate that 'fresh' (and perhaps authenticity and exoticism as well) is implicated in continual, but ultimately futile, efforts to present an 'ethical' self. Recognizing the importance of Johnston and Baumann's contribution, I offer this thesis as a complimentary, rather than competing, analysis.

The final piece of this literature review looks specifically at the notion of 'fresh,' which emerged as a core theme in my data and is the focus of my analysis. In the following section, I look at existing work that addresses 'freshness' as a valued marker of cultural consumption.

\section{Fresh}

In her recently published book, Fresh: A Perishable History, Suzanne Freidberg (2009) offers a comprehensive analysis of how 'freshness' is valued in the modern context. Freidberg notes that there is one question that consumers continually return to when making choices about food; we ask: "Is it fresh?" Freshness is perceived to ensure quality, optimize pleasure, and prevent disappointment and danger, but what does fresh actually mean? In her book, Freidberg explores how notions of freshness shift as consumers become increasingly removed from the day-to-day work of growing and preparing food. 
Freidberg begins her investigation by citing an official public inquiry, hosted by The Food and Drug Administration (FDA) in July 2000, that aimed to discuss and lay down guidelines for "the use of the term "fresh"' (2). Quoting a lobbyist who asserted that 'fresh' is "the cornerstone" of the food industry, Freidberg points to both the difficulties and significance of defining this term. She outlines a series of questions that were addressed in this inquiry:

Could freshness be measured? Should it be defined by taste or by internal qualities? Did the agency need another term to describe foods that were processed but 'fresh-like'? How much information about a product's freshness did consumers need or want on a label? And finally, if the FDA redefined the legal meaning of "fresh," who would profit or lose out? (Freidberg, 2009:2)

As Freidberg notes, the last question was of primary concern to those who attended the meeting (and for those in the food industry at large). While 'fresh' was first defined simply as any food that had not been frozen, heated, chemically treated, or "otherwise preserved," this definition quickly fell short, failing to accommodate key exceptions. Freidberg offers a number of examples that illustrate the complexity of the fresh label: "fresh" pasteurized milk, "fresh frozen" vegetables, "fresh cooked" seafood, "fresh squeezed" juice, and any number of "refrigerator fresh" perishables. All of these usages of the word 'fresh' problematize any basic definition of freshness that could serve as an overarching point of reference for industry or consumers.

In order to probe the complexities of this term, Freidberg breaks her analysis into chapters devoted to single food products. "Tracing the history [of fresh] by way of a tour through an ordinary refrigerator" (3), she explores notions of freshness as they relate to beef, eggs, fruit, vegetables, milk, and fish. Freidberg observes that "fresh" has come to 
mean very different things for different foods. She notes that whereas a producer of bagged salads might describe fresh as anything "alive and respiring," this hardly suits as a working definition of freshness for poultry (2). Whereas fruit may be valued as "fresh" based on the vibrancy of colour, an egg conceals its freshness (or lack thereof) beneath an unrevealing white shell. Whereas the best fresh-caught fish is served immediately as a "catch of the day" (because of enzymes and bacteria that make fish smell and decay), grade AAA fresh beef is hung for weeks (a process of aging that tenderizes the animal's flesh) before it is served. Freidberg demonstrates that the meanings of fresh varies hugely, making it arguably the most complex and contested of food qualities. Using Freidberg's work as a guide, I explore these complexities and point to five core layers of 'freshness' that emerged in my data: local, seasonal, non-contaminated, non-packaged, and human handled. By looking at how my interviewees articulated these notions of 'fresh,' I am able to point to the sociological implications of this food value in our contemporary Western food system.

Freidberg charts the evolution of a food system that has seen dramatic changes in the last century by looking at how 'fresh' is applied to different food groups. The emergence of refrigerated transport vehicles has transformed the beef industry, chemical stabilizers slow the decay of fruit, and fish sedatives allow for the global trade of 'fresh' seafood. While these technological 'advances' - these inventions and innovations of our industrialized food system - signify an unmistakable move away from freshness in any traditional sense (i.e. the guaranteed 'freshness' of food produced through local, small- 
scale subsistence farming), they do create a system in which the possibility of freshness seems more attainable, and more desirable, than ever. Similar to Freidberg, I believe the concept of 'freshness' holds a particularly unique, and significant role as a core urban food value. It reflects a suspicion of, and distaste for, highly processed, 'value-added' food commodities that threaten the safety and quality of our food, while simultaneously celebrating technologies of food preservation and packaging that promise to highlight and enhance the freshness of the food.

Freidberg (2009: 3) states that the appeal of 'fresh' lies in 'the anxieties and dilemmas borne of industrial capitalism and the culture of mass consumption." In this thesis, I investigate the appeal of 'fresh' for my research participants and explore how individuals' engagement with the notion of 'freshness' functions as a core dynamic of contemporary food consumption. My analysis of 'fresh' emerges directly from data gathered from participant observation research at The Green Door Restaurant and indepth interviews with 15 patrons. In the next chapter, I outline the specifics of my research design. 


\section{CHAPTER 3}

\section{RESEARCH DESIGN}

This thesis is concerned with identifying the values that inform urban food choice and with exploring the sociological implications of these values on the individual consumer. In this chapter, I outline the research design that has allowed me to identify 'fresh' as a core signifier of 'good' food and develop an analysis that contributes to existing food studies literature. The most significant feature of this research design is my use of The Green Door Restaurant as a site of study. Drawing on a grounded theory approach to data collection and analysis (Charmaz, 2006), I employed ethnographic field observation and in-depth semi-structured interviews to explore how food choices are enacted in this space. My intention has been to gather enough data about the persons, processes, and setting of The Green Door to understand the nature and range of food values that are perceived and experienced there. Through this focused study, I am able to explore how 'fresh' functions in the lived experience of dining out. I argue that The Green Door constructs a 'fresh' space, which appeals to consumer desires and exemplifies contemporary dynamics of status display. This research is significant because it points to a critical breakdown in the presentation of self for modern consumers, and highlights the failure of consumption-based solutions to address the systemic inefficiencies and inequalities of our contemporary food system. 


\section{Sample Selection}

My selection of The Green Door Restaurant as the focus of my research project was made according to the principles of "information-oriented selection;" it was based on my expectation that this restaurant would serve as a particularly rich source of information. This selection strategy allowed me to "maximize the utility of information from a small sample" (Flyvberg, 2001: 79), which was imperative in consideration of the relatively narrow time frame for a Master's thesis.

The Green Door Restaurant emerged as a particularly attractive site of study because it explicitly incorporates, in its signage, customer 'guide' (Guide to The Green Door, 2010), and website (www.greendoor.ca), many of the words that are already receiving scholarly attention as value-laden markers of contemporary food discourse. This restaurant makes an explicit commitment to serving 'simple,' 'wholesome' food (Johnston \& Baumann, 2010). It specializes in 'healthy' foods made from ingredients that are locally grown, but brought to life by exotic flavours. Much of the food is vegan or gluten-free; ingredients are 'natural' and 'organic' (Guide to the Green Door, 2010).

The Green Door also constructs a unique dining space. Specifically, the restaurant employs a serve-yourself, pay-by-weight, buffet-style format and provides a cafeteria-style setting for eat-in dining. I was interested in investigating the social implications of this layout; it struck me that this seemingly more egalitarian dining system is significant considering the relative affluence of the clientele. I predicted that this egalitarian atmosphere might reflect consumer efforts to seek 'alternative' food 
consumption practices and flagged this restaurant as a potentially rich site of food choice behaviour. In carrying out my research, I have found that these structural elements of the restaurant reflect, and have provided a unique opportunity to further investigate, tensions between democracy and distinction that characterize contemporary food practices (Johnston \& Baumann 2010).

The casual, open-concept format of the dining space also made this restaurant a particularly good site of study for ethnographic field research. Because of the close proximity of tables, I was able to make detailed observations about what patrons were doing and saying to one another over the course of their dining experience. Regardless of where I sat, I occupied a good position to observe the full scope of activity, from food selection at the buffet to consumption to dish drop-off. The communal atmosphere also allowed me to engage in informal interactions with other patrons even in the initial stages of research (before I began my interviews), which gave me insight as to what Green Door patrons value in food, and how these values fit in to their presentation of self ${ }^{3}$.

The Green Door Restaurant is a well-known, busy dining destination that has been in business for over 20 years. As many of my interview participants indicated, The Green Door is regarded as a staple in Ottawa and, as such, may be understood to reflect a

\footnotetext{
${ }^{3}$ Ethical considerations related to engaging in 'covert' research at The Green Door were relatively minimal because restaurants are public sites where expectations of privacy are low. Due to my concern about the privacy of Green Door customers who would not be able to be made aware you were conducting research on them and their activities, 1 met with Leslie Macdonald-Hicks of the Carleton University Ethics Board to ensure I would be aware of all of the ethical protections required of this type of research approach (i.e. not identifying patrons by name in my analysis, not making audio or video recordings of interactions, etc). My field observations did not subject patrons to any risks that they would not otherwise be subject to at The Green Door.
} 
viable style of urban food consumption. For these reasons, I predicted that The Green Door would serve as a rich and relevant site of exploration for my research. I correctly anticipated that this site would serve as a concrete starting place from which to move forward with an exploration of emerging food values.

My research design also required that I develop a selection strategy for interview participants. As an initial recruitment strategy, I posted a call (see Appendix A) on the Ottawa Foodies blog (www.ottawafoodies.com). I targeted this group to draw on a sample of local individuals who would be able to articulate, to some degree, how they value food. This sampling decision was motivated by an attempt to ground my thesis in the body of sociological work currently being done on 'foodie' discourse (Johnston \& Baumann, 2010). My intention was to explore how individuals participating in a 'Green Door experience' relate to the performance of a 'foodie' identity and to explore questions of democracy, distinction, anxiety, and responsibilization of the individual, that relate to this discussion.

Although I received some response from this posting, my sample was ultimately determined by a more effective response to subsequent recruitment postings that targeted a broader audience. Specifically, I posted flyers at a number of grocery stores and cafes that similarly draw on notions of 'organic,' 'local,' 'healthy,' or 'alternative' food in their corporate branding. I predicted that the Green Door patrons I was targeting might also frequent these retailers. I also sent out an email across my personal networks in a final attempt to recruit participants. The interview posting called for people who were 
interested in urban food consumption, passionate about food, and had been to The Green Door. Interviewees' self-identification with these characteristics merited their inclusion in my study; I interviewed all individuals who responded to my post.

Although not all of my research participants were affiliated with the Ottawa Foodies blog, I argue that my sample does allow me to engage with Johnston and Baumann's work on gourmet food discourse. All of my participants articulated an attitude towards food that reflects the privilege of middle-upper class Western urban living; respondents were not concerned with finding enough to eat, but with choosing 'good' food from an abundance of options. By simply going to The Green Door, they demonstrated their interest in engaging in 'alternative' food practices (i.e. vegetarianism), as well as their relative affluence. Specifically, my research participants demonstrated an enthusiasm for food knowledge and discerning tastes that aligns with Johnston and Baumann's work on 'foodie' consumption practices. I anticipated this compatibility through my initial field observations at The Green Door and targeted Green Door patrons as my interview sample specifically because of these characteristics.

In the end, my interview sample consisted of 15 middle-class individuals, aged 25 to 60 , with varied backgrounds and interests. To offer a general impression of the sample, I interviewed a soon-to-retire elementary school principal, a 26-year-old businesswoman, an actor, a stay-at-home mom, a soldier in the Canadian Armed Forces, a raiki master, and a number of graduate students. It is relevant to note that simply because of the responses I received, my sample included only two men, and did not 
include any visible minorities. I do not view this as problematic; I did not make a concerted effort to create any proportionally reflective assembly of Green Door clientele, and I do not claim to offer a representative sample. However, I suggest that my sample does, in general terms, reflect typical Green Door clientele in that research participants were educated, had access to disposable income, and demonstrated a personal interest in choosing 'good' food. I have assigned each interviewee a pseudonym to maintain their anonymity in my analysis.

\section{Data Collection}

I needed to gather data that would allow me to understand how food values are constructed within The Green Door Restaurant and how their patrons engage with these values in their food practices. In order to do so, I engaged in several months of ethnographic field observation at The Green Door and held 15 in-depth semi-structured interviews with Green Door patrons who self-identified through the posting tactics described above.

Because the basis of my research design was to study food choice within one particular site of food consumption, my first task was to establish a detailed understanding of The Green Door Restaurant. Schatzmann and Strauss (1973) offer useful instructions regarding the logistics of entering a research site. They advocate first 'casing' the location so as to determine the suitability and feasibility of 'using' the research site (19). I found that the preliminary 'casing' I engaged in was immensely useful. Extant texts, including the restaurants' websites, menus, community boards, and 
cookbooks, served as a primary resource during this 'casing' phase. Charmaz (2006) asserts that extant texts - varied documents that the researcher uses as data, but has had no hand in shaping - are a valuable source of data because of their relatively unobtrusive availability and capacity to reflect shared perceptions. In my study, these texts served as 'official messaging' of Green Door management, and were particularly relevant for my research because they help shape consumers' perceptions about the food choices they make in this space.

By familiarizing myself with some of the practices of The Green Door through an examination of this textual material, and reflecting on my informal visits to The Green Door as a patron, I was able to confirm that this restaurant would serve as a viable focus of study and establish a basic understanding of the practices and processes that shape food consumption in this space. To proceed with more involved ethnographic field observation, I acquired written permission from a manager of The Green Door to conduct research at his restaurant. Informing each individual patron was not necessary, as I did not identify any of the patrons I observed in my analysis or subject them to any additional risks, but gaining permission from the owner was an important step in fulfilling the requirements of the Carleton University Ethics Board and legitimizing my presence in the space.

Once I entered the research site, my basic task was to "acquire an appreciation for the nature of life" at The Green Door (Emerson et al, 1995). Because it was my intention to explore choice within the context of a specific restaurant, it was necessary for me to 
first establish a nuanced understanding of how the space is constructed and the practices and processes that go on within it. I entered into this research with the simple question, "What is happening here?" (Glaser, 1978). I took notes on everything I observed, paying particular attention to how people chose their food and how they behaved toward one another while selecting and eating it.

Throughout my time in the field I tried to remain as open as possible to whatever I saw or sensed (Charmaz, 2006: 14). I began by recording "concrete sensory details" about the basic scenes, settings, objects, people, and actions I observed (Emerson et al. 1995:68). Flyvberg advocates focusing on the particular, "the minutiae, practices, and concrete cases" (2001: 136) to access the richest data. This methodology entailed recording extensive field notes on the physical construction of the space, including the interior design, furniture, dinnerware (mugs, plates, cutlery), artwork, lighting, and music. I was also concerned with observing and recording how the layout of the space directs movement through the restaurant and how individuals act and interact within the construct of this space.

During each field visit I wrote several pages of notes by hand, allowing my natural observational impulses to direct my focus. For example, if a painting caught my attention, I would spend some time taking notes on the art on the walls. If a piece of conversation caught my ear, I would record it. Similar to Snow and Anderson (1987), I found eavesdropping to be a particularly rich source of data. Because of the social nature of dining for many patrons, dialogue between groups and individuals is a major 
component of the Green Door experience. "Listening to others within a bounded interactional encounter without being a part of that encounter" (Snow \& Anderson, 1987: 8) provided me with valuable access to the relatively unselfconscious verbal exchanges of participants. It was particularly possible to gather this data because of the close proximity between all Green Door patrons.

Occasionally I was also able to engage in informal conversation with other patrons. Immediately following such an interaction I would attempt to record it verbatim, usually staying on site to allow the context of the exchange to trigger additional details in my memory. Once at home I elaborated on the jot notes I took in the field, fleshing out my short hand descriptions into a clarified narrative. I also wrote a series of memos, musing over and expanding on themes that emerged from my observations. Memo writing provided me the opportunity to become "actively engaged in [my] materials, to develop [my] ideas, and to fine-tune [my] subsequent data-gathering" (Charmaz: 2006, 72).

My growing familiarity with The Green Door Restaurant, as I went back again and again, served as the foundation for my analysis, shaping my ideas about what happens in the space and informing the questions I would later ask my interviewees. Ethnographic field observation allowed me to immerse myself in the inner-workings of The Green Door to begin to construct an analysis that explores, as fully as possible, "the situational contours and contexts" they construct (Mason, 2006: 16). Once I acclimatized myself to the practices and processes of this restaurant, I proceeded with a second phase 
of data collection and analysis: 15 formal in-depth semi-structured interviews with Green Door patrons.

Interviews are a particularly useful research method for exploring how a particular topic is perceived or experienced (Charmaz, 2006; Lofland \& Lofland, 1984, 1995). This methodology was relevant to my research because I wanted to explore the implications of food choice on individuals and on personal identity. Talking to patrons directly about how they experience food practices at The Green Door allowed me to explore what meanings different participants attribute to the processes and practices that they engage with in this space and how these processes and practices reflect or conflict with their ideas about who they are. "What meanings do participants attribute to [the topics]? How do they talk about them? What do they emphasize? What do they leave out?" (Charmaz, 2006: 20). These were the types of questions that drove my research agenda.

In accordance with the grounded theory approach, my interview script consisted of a few broad, open-ended questions about participants' personal ideas about food and their participation in Green Door dining (Charmaz, 2006: 26). I asked: What kind of an eater are you? What makes food 'good'? Why do you go to The Green Door? How would you describe food consumption in Ottawa? And how would you describe The Green Door? These broad questions allowed me to learn not only about patrons' relationships to The Green Door, but also about the broader context of food ethics and practices that shape and frame them. I also prepared a series of follow-up, or probing, 
questions that could invite research participants to expand or go in to more detail, on their answers (see Appendix B for full interview script). Although I gave my interview participants permission to ask for clarification if they felt they needed it, I invited them to follow their instincts in responding to my questions and to trust that their answers would be relevant. Because I did not enter into my research with specific themes to 'test' for, any ideas that emerged organically from the interview questions could serve as the data from which I could develop my analysis.

I recorded all interviews on audiotape in order to capture the interactions verbatim for transcription. Recognizing that the nuances of the interviews may not be transmitted in the recording, I also made short notes during the interview, which I could then expand on afterwards to preserve my immediate reactions and flag my memory on certain points during later analysis. Recording data from the interviews, including my reactions, in as much detail as possible served to minimize any distortions that might have occurred in later stages of data analysis as a result of my simply relying on memory or vague impressions. This said, I understand that interviews are dialogical in nature (Flyvberg, 2001: 139) and inevitably reflected my subjective involvement in the interaction. The interview narrative is always a discursive process between the interviewer and the interviewee (Enosh \& Buchbinder, 2005) and therefore the data I collected and the analysis I developed inevitably reflect my participation in the research.

More broadly, I acknowledge that my analysis reflects my deep interest in the social meanings of food practices and my belief that these practices have significant 
sociological implications. Like the patrons I interviewed, I am passionate about eating and am interested in the political and environmental ramifications of my food choices. This enthusiasm no doubt had an impact on the tone and direction of the interviews I conducted. However, my data collection has been extensive and my analysis rigorous; I engaged in the cyclical processes of data collection and analysis to ensure that the analysis I present in the following chapters emerged from, and remains rooted in, my data. Further, I have attempted to be as transparent as possible about the particularities of my research practices (including how particular themes emerged) as well as the limited scope of the study. Because I established these safeguards, my personal interest in the subject should not be considered a liability, but rather an asset (Flyvberg, 2001).

While I had initially considered attempting to do all interviews at The Green Door, I ultimately decided against it due to space constraints (taking up tables in the restaurant), noise issues, and financial limits. Although it would have been interesting to observe my interviewees' behaviour while 'in the field,' I believed that being in the setting might also affect how freely they felt they could speak about their feelings and impressions. I compensated for this decision through my field observations. The sensitivity I developed through conducting fieldwork gave me an understanding of the processes described in the interviews. My nuanced familiarity with how The Green Door is constructed and operated, allowed me to understand interviewees' references and probe their responses for further clarification when necessary. I argue that it was not in fact necessary to observe my interviewees as they dined at The Green Door because my initial 
observations allowed me to construct a solid understanding of the values being enacted at The Green Door. The interview phase of my research allowed me to learn about patrons' perceptions of these values so that I could better understand how they are implicated in processes of self. All interviews lasted approximately one hour.

In sum, this thesis draws on data compiled through two months of weekly field visits, 15 interviews with Green Door patrons, and a variety of published Green Door material, including their website, cookbook and a 20-page 'Guide to The Green Door' information brochure. Importantly, I went back and forth between these sources throughout the data collection process, alternating between data collection methods to explore emerging themes as comprehensively as possible. I referred to extent texts throughout the data collection of this project, and returned to The Green Door repeatedly over the course of the interview phase of data collection in order to again observe and reflect upon whether the space and activities described by my interviewees matched the types of observations I had also been noting. Weaving these research methods together allowed me to collect a detailed and comprehensive mass of data and advance my sympathetic understanding of the meaning that the Green Door held for its patrons. Specifically, my intention was to gather enough data about the persons, processes, and setting of The Green Door to understand the nature and range of food values that are perceived and experienced there. I attempted to be as detailed as possible in gathering my data in order to "reveal what lies beneath the surface" (Charmaz, 2006: 19). These 
data provided the raw material from which the data analysis described in the following section emerged.

\section{Data Analysis Strategy}

Over the course of a year, I compiled hundreds of pages of field notes and transcripts. Confronted with this mass of data, I engaged in a systematic analysis that involved three stages of coding. Through this process, I identified 'fresh' as a core value of urban food choice and constructed an analysis that illustrates its significance to individuals' presentation of self and the broader dynamics of our industrialized food system.

Following the grounded theory approach described by Charmaz (2006), I started with a process of 'initial coding,' focusing on fragments of data, which can be categorized into segments and summarized concisely (Charmaz, 2006: 52). Specifically, I engaged in a process of line-by-line coding, 'naming' each line of my written data (Glaser, 1978). This methodological strategy allowed me to reflect on my data in considerable detail, while also encouraging me to crystallize and communicate the essential meanings of fully developed sentences (Charmaz, 2006: 50). My intention was to have codes reflect the content of each interview line as closely as possible rather than pre-create or force codes upon my data.

Once this initial coding process was complete, I repeatedly reviewed all of my emergent codes and highlighted patterns to familiarize myself with the general categories that had become apparent. Taking a copy of the field notes I gathered during my initial two-month phase of direct field observation, I began a process of cutting out lines or 
sections of the coded text and physically grouped compatible codes into organized piles. I adopted this analytical strategy in order to see the tangible emergence of prominent themes, as well as to look at how the commonly themed component parts of my data might interact outside of their original context. Many of these specific categories informed my interview questions and re-emerged as significant elements of my final analysis.

Taking the core themes that emerged from this stage of the data analysis, I moved on to the 'focused coding' stage (Charmaz, 2006: 42). Focused coding entails selecting initial line-by-line data codes that seem to be most useful and testing them against more extensive data (42). Thus, I went back through all of my data to look for content that related to, or exemplified the broader thematic codes that had emerged during the line-byline coding process. I pulled data sections from their original contexts and compared and contrasted the data as thematically-organized groups. This strategy allowed me to reveal phrases or data sections that were contradictory or antithetical as well those that I could merge together into broader themes. Specifically, I used the methodological strategy of focused coding to explore how commonly themed component parts of my data could interact and 'stand up' outside of their original context.

It is important to clarify that I began analysis from the moment I began collecting data. This was particularly critical to the legitimacy of my grounded theory-inspired study, because it ensured that the trajectory of my research was determined by my data from the start, and that it remained consistently in line with the sentiments of my data as I 
developed my analysis. Grounded theory practices are based on inductive reasoning through a cyclical process of data collection and analysis (Charmaz, 2006). Throughout my research, I worked back and forth between data from my field notes, interview transcripts, and extant texts to engage in what Glaser and Strauss (1967) term the 'constant comparative method.' Thus, for example, I asked: What makes the Green Door distinct from other restaurants? What are the particularities of the food/the clientele/the décor from one week to the next? How are these elements perceived by patrons? How does the lived experience of patrons compare with the official mandate of The Green Door, as outlined in The Guide to the Green Door? How do my interview responses align with or contradict customer behavior as I observed it? How does this differ from week to week? Day to day? Patron to patron? This allowed me to develop an analysis that captured the particularities of my study in rich detail and emerged organically from my data.

It is relevant to note that the content of my analysis, as it appears in the following chapters, differs considerably from my original ideas about the direction of my research. When I first entered the research site for the direct observation phase of data collection, I predicted that my analysis would focus on how The Green Door space shapes food consumption practices. For example, I wondered if the cafeteria-style construction of the dining space affects patron interaction in a specific way, if the alternative pay-by-weight system meaningfully impacts how patrons valued food, or how the open boundaries between the kitchen and front of house space influence patrons' dining experiences. I 
predicted that I would discover how the unique characteristics of The Green Door space relate to patrons' perceptions of a personal food identity. However, in carrying out the initial direct observation phase of data collection, I became drawn to food choice as a central focus for my analysis (see Appendix C). I saw that making 'good' choices was an important component of Green Door dining, and I became interested in exploring how this might reflect the dynamics of food choice for these patrons in the broader context of their day to day lives.

The result of this shift in focus to individual food choice is that my analysis explores aspects of contemporary food consumption that I had not anticipated. Specifically, the content of my interview data has directed me to address food politics and to consider if, and how, dining out can be framed as a viable strategy for social change. Because food identity, rather than food activism, has been the primary focus of this thesis, I do not explore the complexities of social movement theories or interrogate this food activism layer of my data in as much detail as this topic merits. Rather, I focus on how notions of ethical food consumption, which are importantly connected to food security activism, are implicated in the presentation of self. I see that the additional analytical possibilities related to social movement strategy and political food activism are a testament to the richness of my data and I hope to pick up on these issues in subsequent research.

Throughout all stages of the research process, I immersed myself in the data and engaged intellectually with potential ideas and emerging themes through the practice of 
memo writing. According to grounded theory practices, memo writing is the "pivotal intermediate step between data collection and writing drafts" (Charmaz, 2006: 72). From the first time I entered the field, writing memos became an important means through which I processed and examined the data content produced by my research activities. I wrote memos throughout the research process to cultivate an active and expansive analytical engagement with core categories and key themes. Ranging from stream-ofconscious reactionary writing to more methodical musings on a certain theme, memos have served as an invaluable source of reflection and direction throughout my research process. To offer an example, the following is an excerpt from a memo I wrote early on in my fieldwork called, 'The Plate: The Other Party:'

At nearly all tables I see plates loaded with different dishes, all flavours mingling and at many of these tables people methodically combining small pieces of different dishes on their fork for a particular bite. It seems that an important activity in this social space is the act of Creating Bites. Dipping bread in soup. Twirling noodles on the fork and then stabbing a small piece of avocado onto the end of the fork. There is personalization in the primary selection of constructing a plate, but there is also secondary selection in constructing a fork-full. Creative Eating. What is the logic, on the part of the individual, that informs this business of eating (combining dishes, cutting incompatible shapes to fit neatly on a fork together)? Is this different at The Green Door because of personalized plate construction?

This memo illustrates my near-immediate recognition that patrons of The Green Door engage in reflexive decision-making throughout all stages of their food practices. Having begun to interrogate these types of initial musings in memos, I explored these observations further in my interviews, and eventually identified 'fresh' as a core food value that informs reflexive decision-making and food practices. 
I arrived at the notion of 'fresh' through this cyclical process of data interrogation, first noting the prevalence of the term 'fresh' throughout my data, and then making links to categories and characteristics within my data that contributed to my understanding of this concept. I began this research project by thinking about the 'big picture' of food consumption, considering the particularities and dilemmas of the Western urban food system, and then narrowed in on 'choice' as a particularly prominent issue. Using The Green Door as a focus, I attempted to identify the values that inform food choice for urban individuals. I discovered that five categories, which emerged through a process of focused coding, belonged within a broader umbrella-category of 'freshness:' local, seasonal, non-contaminated, non-packaged, and human handled. I recognized that these categories could be understood as interconnected component parts of individuals' personal food ethics. I have developed an analysis that illustrates how these food values are enlisted by urban consumers as they make choices about food from within the industrialized Western food system and, in so doing, perform self. I illustrate the complexities of this analysis in full detail in the following chapter.

Despite my heavy reliance on grounded theory practices, it is necessary for me to clarify that the conclusions I draw in my data analysis cannot ultimately be understood to reflect a 'grounded theory' study. In order to make this claim, I needed to engage in an additional phase of theoretical sampling, which would involve going back into 'the field' to seek additional data that could be used to elaborate on, refine, and strengthen the core 
categories or themes that had emerged from the original research. Due to time constraints, however, I did not take this final methodological step.

The aim of returning to the field and interrogating the data repeatedly is 'saturation.' Saturation can be declared when categories no longer spark new theoretical insights (Charmaz, 2006: 113). Once categories have achieved 'saturation' they are sorted, mapped and diagrammed into the analytical frame that ultimately constitutes the researcher's theory (121). Within the scope of a Master's thesis, this extremely timeintensive process was not possible. In consequence, although grounded theory served as my primary guide as I designed and executed my research methodology - and my concept of 'fresh' emerged organically from my data - my findings must ultimately be understood as a thematic analysis, rather than a fully realized grounded theory study.

\section{Considerations for Qualitative Research}

The strength of qualitative research is that it allows the researcher to access and capture the multi-dimensionality of the 'lived experience' (Mason, 2006: 11). Achievement of this goal is immensely important for my research study because food consumption practices constitute a complex and profoundly important component of the 'lived experience.' The complexity of this every-day life practice is reflected in my layered analysis of how food choices are implicated in consumers' attempts to present a responsible, 'ethical' self by consuming 'fresh.'

There are, however, a number of considerations that I had to address as I entered both the data collection and the data analysis phase of my research study. Firstly, I 
recognized that my background assumptions and disciplinary perspectives would inevitably sensitize me to particular possibilities and processes in my data. Blumer's (1969) notion of 'sensitizing concepts' captures the tendency for researchers to begin their studies with certain initial ideas to pursue and questions to ask. From the start, I anticipated looking at how The Green Door serves as a site of identity management. I was interested also in examining how much crossover exists between self-sustaining food practices (such as dining at a healthy, pesticide free, "socially responsible" restaurant) and larger efforts for community food security. I intended to pay particular attention to how The Green Door fits into a larger network of 'sustainable consumption practices' within Ottawa (i.e. community gardens, social movement cinema, holistic medicine). Importantly, I recognized that these sensitizing concepts are "a place to start, not to end" (Charmaz, 2003: 17). In consequence, my use of a grounded theory approach was instrumental in ensuring that the data, rather than my pre-conceived notions, directed my analysis. Although some of my initial thoughts have remained relevant (i.e. as subjective beings we can never fully remove ourselves from the research process or our data), my analysis has taken me down unexpected avenues and into an area that I had not anticipated.

Secondly, I knew that I could not enter this research project with an objective perspective or "hold a privileged position from which the final truth can be told" (139). Flyvberg (2001) argues, however, that no social science researcher can, nor need to, assume an objective perspective. Rather, the researcher must allow his/her values to aid 
in producing social science research "matters" (166). Flyvberg claims that personal interests and experiences can be viewed as assets, rather than liabilities. Reflexivity, or "recognizing the social location of the researcher" is a crucial component of qualitative research (Doucet \& Mauthner, 2003). It is important that the researcher include a practice that will allow him/her to recognize how pre-conceived notions and personal biases factor into and affect the research process. Thus, it was important that I made explicit my position (socially and emotionally) in my research both to myself and to my research participants. As such, I acknowledged as best I could at all stages of the project that a passion for food directed me towards this area of research, and that my analysis would inevitably reflect my interests in alternative urban consumption practices. I made sure to write memos on my personal observations and reactions through all stages of data collection and analysis to monitor, and make explicit, my position in the work.

Lastly, the question of generalizability tends to haunt the qualitative research agenda, especially in the face of positivist and post-positivist authority (Charmaz, 2006). A prominent critique of qualitative research is that results are "too contextual" and therefore are not generalizable (Mason, 2006: 17). This thesis has been constructed on the widely shared belief that there is immense value in exploring the context-dependant particulars of a social space (Flyvberg, 2001; Smith, 1999; Charmaz, 2006). While some scholars are not concerned with attempting to draw generalizations from their work (Flyvberg, 2003), others argue that "generalization seems worth pursuing if at all possible" (Seale, 1999: 107). Seale asserts that results from a qualitative case study can 
be transferred to other cases if the 'sending' and 'receiving' cases share key contextual characteristics (117). I believe that my analysis may be 'transferable' to other cases that explore the food consumption practices of middle to upper class urban Canadians and/or investigate food values (such as local, organic, seasonal, non-packaged, and human handled) that have emerged in contemporary Western food discourse. In addressing these issues, this study may prove to hold a wider relevance in food studies literature.

I suggest that my analysis of The Green Door and its patrons is transferable within a larger sociology of food because it identifies and engages with key concepts and characteristics that recur as cornerstones in much of the contemporary discourse existing currently in the sociological literature on food. By exploring these issues, I offer my analysis of 'fresh' and my discoveries of The Green Door and its patrons as part of the wider complexity of social behaviour involved in food consumption in the Western urban foodscape. 


\section{CHAPTER 4}

\section{ANALYSIS I: The Value of 'Fresh'}

The goal of this thesis is to explore the values that inform food choice in a contemporary urban food system. Based on data gathered through participant observation and 15 indepth interviews with patrons of the Green Door restaurant, I argue that choices about food constitute a particularly significant aspect of urban life and seek to explore the sociological implications of food values as they are enacted in consumer choice. Specifically, this thesis is concerned with understanding how food values are implicated in processes of identity management, and what they say about the state and direction of our contemporary food system.

Over the past year, I engaged in several months of ethnographic field observation at The Green Door Restaurant and 15 interviews with Green Door patrons to explore these dynamics. My data analysis indicates that 'fresh' is a core food value for my interview participants and that these individuals engage with 'fresh' in efforts to present a 'valued' self. This finding is significant for a number of reasons; it exemplifies a critical breakdown in processes of self for the individual, whose efforts to present a responsible, 'ethical' self are ultimately undermined by their acknowledgment that the urban food system is fundamentally unsustainable and inequitable, and demonstrates that these issues cannot be challenged through individual efforts of 'ethical consumption.'

In this first analysis chapter, I introduce 'fresh' as a core food value and a core concept of analysis in this thesis. Drawing upon interview responses, I identify five 
layers of 'fresh,' each of which stand for food that is 'as close to its natural state as possible' (Guide to the Green Door, 2009) by being: (1) local, (2) seasonal, (3) noncontaminated, (4) non-packaged, and (5) human-handled. Each of these qualities was articulated by my research participants as an important component of their personal food ethic. However, none of the participants perceived they were able to attend to all of these values in all of their food choices. My data analysis shows that these consumers navigate between these five food values, often making allowances in one area to achieve fulfillment of another food value, to ultimately create an overall diet that is 'good enough.' I will demonstrate that my interviewees see effective achievement of this goal as a significant challenge and that they explore a vast range of information sources in their attempts to construct a personal food practice that addresses their ideological concerns and meets their individual needs. Ultimately, as I illustrate in the following pages, these efforts are implicated in processes of self for the contemporary urban individual; the individuals in my study engage with 'fresh' in concerted and continual efforts to present a responsible, 'ethical' self.

\section{De-constructing the 'Fresh Food Ethic'}

As I engaged in the focused coding of my interview data to identify core food values, I was struck by the fact that each participant in my study expressed a personal food ethic that incorporated a massive number of specific and varied priorities. 'Good' food was perceived to be animal-free, raw, live, gluten-free, salty, high in protein, organic, meaty, low in sodium, local, comforting, not-packaged, 'natural,' nutrient dense, and/or unusual, 
to name only a few of the qualifiers used. Interviewees also described food consumption practices that involved decision-making about a wide range of food-related issues. For example, Tina ${ }^{4}$, a graduate student who is vegan and runs a two-acre vegetable farm with her partner, summarizes her decision-making process:

There's like, this whole list of criteria, I guess that are kind of like ethical, or moral considerations. Or you know, like, yeah, like worker rights, that type of thing. Kind of like social justice framework. Or an environmental framework. Um, you know we have like an industrial [system], so I think questions of scale are important in terms of, like, sustainability. But then the other side is like, that is like, it has to be Good. Like, it has to taste good.... Like if it's local, if it's organic, if it was living wage, whatever, if it tastes like crap, then I don't think that's enough.

In this passage, Tina experiences a 'moral' dilemma where she balances potential exploitation of food workers, environmental degradation and pollution, and her desire for sensual and nutritional gratification. Because food is meaningful in a multitude of capacities - politically, ecologically, socially, sensually, and nutritionally - Tina is required to balance a 'whole list of criteria,' obtaining food from particular sources and using particular food practices, to express an ethical self. This quote illustrates that Tina's ethical/moral dilemma, and her sense of maintaining an ethical 'food' self, involves a decision-making process in which she balances her personal needs/desires with her sense of justice regarding how food is grown, harvested, produced and packaged.

The last line of Tina's quote reveals the crux of the matter. Recognizing the risks of making 'bad' food choices and the difficulty in finding foods that satisfy every

\footnotetext{
${ }^{4}$ All the names that appear in my analysis are pseudonyms to ensure the anonymity of my interviewees.
} 
qualification, what is it that makes a food choice good 'enough'? Regardless of the individual's personal food ethic - each distinct in terms of how the individual weighed and prioritized specific nutritional, ethical, environmental issues - our discussions would inevitably return to one key idea. Good food is fresh. As Ann articulated in her first response: "I would say, in general, I like to eat things that are fresh." More than just another qualifier to be added to the list with lactose-free and high-in-fibre, my data indicate that freshness is a uniquely complex and meaning-filled word within contemporary food discourse. From the perspective of my interviewees, few food qualities seem as "unquestionably good as freshness" (Freidberg, 2009: 2). Fresh is attractive. Fresh is healthy. Fresh is safe. Fresh is ambiguous. Fresh is powerful because it encompasses the political, environmental, ethical, nutritional, and sensual concerns of the urban individuals I interviewed.

In the sections that follow, I explore what it is about 'fresh' that makes it an overarching priority for my interviewees and investigate how eating 'fresh' food contributes to one's sense of self as an ethical eater. Specifically, I will illustrate how each of the core food values I identified in my interview data - local, seasonal, non-contaminated, non-packed, and human-handled - connects to the broader narrative of 'freshness' described in the accounts provided by the fifteen people interviewed for this research project. 


\section{Local}

Local food is 'fresh' because it implies a spatial 'closeness' between food and its 'natural state' on the farm, in the lake, or off the road where it was grown. Freidberg (2009) points out that, for most of human history, fresh or perishable foods were by definition local (9). Before the advent of refrigeration, food was 'fresh' simply because it could not be transported very far before it spoiled. Now, despite innumerable innovations in technology designed to free us from the limits of local production, an association between local production and freshness remains entrenched in public perceptions of 'good' food. For the individuals I spoke with, local sourcing is a cornerstone of their food consumption decision-making and has innumerable benefits. As one interviewee said, "Local food is important. Like, buying things that are local is definitely important to me."

Erin, a soldier in the Canadian military, speaks to the link between local food and freshness and explains the financial and social benefits of choosing local. When asked what makes food good, she said:

Local for sure. I mean, it means that the food's fresher, it means that transportation costs are lower, so product is cheaper. Plus, you know, it supports the local economy. So yeah, local is really important I think....

Sharing Erin's dedication to supporting local food producers, another interviewee added that supporting regional food production is important because it is sustainable. He uses the rural areas of the Philippines to contrast what he views as our overly-homogenized, and therefore unsustainable, food system:

Every village has a different diet, based entirely on what is a supportable system. They still have their foods systems and everything in place. We eat goats. Why do we eat goats? Because we're in the mountains and we can raise goats and goats 
can live here. We don't ship the goats down to the fishing community, who eats fish, because it's pointless. They eat fish.

In a contemporary Western society where access to culinary variety is valorized as progress, the proposition that individuals limit themselves to only what can be grown in their own region would presumably be viewed as a sentence to insufferable monotony. Indeed, all of my interviewees spoke to the nearly unrivaled importance of having variety in their diets. However, interviewees also insisted that fresh local food offered something that imported products did not. While many interviewees spoke to the fact that buying local is a more ethical choice, they also insisted that local food offers superior taste. For example, Erin qualifies her pragmatic rationale for buying local with an admission that local food tastes better:

When I discovered the market at Lansdowne I just found that everything was better, because it tasted better. So, again being all about how good food tastes, I just go for the local now. For the taste.

When I asked this self-described 'avid' eater to explain her preference for local food, she said, "Well [it's] fresher. A lot of times bigger (giggles) Like, the carrots are huge. Ha! Because they're local." She then went on to tell the story of the time she bought eggs at Costco and ended up throwing out most of the carton " cause they were disgusting compared to the local stuff." She expanded to say that they did not taste like eggs; they tasted "gross," "like wax."

The majority of my interviewees perceive local food as not only the better, but the only edible option. Fresh local food tastes like it 'should;' it tastes real. While technological 'innovations' in mass production offer consumers a uniform aesthetic, 
consistent availability, and low price for staple products like eggs, the food they produce is de-valued by Green Door consumers. It is not 'fresh.' While the actual cause of the waxy, non-egg taste Erin rejected may have been a result of any number of things (the chicken feed used at the factory farm, the chemicals used to prolong the eggs' shelf-life, or even the variety of chicken that had been bred for highest egg production), she understood buying local to be the solution for dealing with the problem of never encountering this type of food experience again.

Interestingly, the value of local food was cemented for Erin when she served on the main base of the Kandahar Air Field (KAF) in Afghanistan. Because ingredients are shipped in from Dubai or Pakistan, "food is never fresh, there's never any vegetables....there's never any good, fresh fruit in KAF." Faced with offerings of rehydrated beef, Erin turned to local food: "The further you go from the main base, the better the food gets, because you get to get local stuff," and she insists, "the fresher it was, the better."

In these types of accounts, I found that Green Door patrons articulated the merits of eating food that has been grown in close proximity to where it is consumed, whether that is within Southern Ontario, the Philippines, or Afghanistan. Clearly drawing on the sentiments of the locavore movement ${ }^{5}$, my interviewees made it clear that choosing local foods is important because it reduces food miles, supports local farmers, is more

\footnotetext{
${ }^{5}$ The local food movement advocates the collaborative development of locally based, self-reliant food economies. 'Locavores' are interested in eating food that is locally produced, rather than transported long distances, in the interest of sustainability and eco-consciousness. This concept gained popularity in Canada with the 2007 publication of The 100 Miles Diet, written by Alisa Smith and J.B. MacKinnon of British
} 
sustainable, and tastes better. Choosing local food responds positively to the dilemma of the 'good' ethical eater who wants to support local business, be environmentally friendly, and reject cruelty to animals and the exploitation of farm workers, while also enjoying healthy, delicious food. For all these reasons, interviewees insist that proximity to source makes food 'good.'

\section{Seasonal}

Local food is not only 'fresh' because of spatial proximity to local farms, but also in its inherent 'closeness' with the natural temporal rhythms of the seasons. Participants also identified eating seasonally as an important priority in their food selection. As Chloe, a vegetarian studying computer science, remarked: 'I'm really careful about where [my food] is coming from and what season things are in." She explains that her love for foods eaten in season, fresh out of the garden, began with her experiences as a child:

We did a lot of gardening...Some of my memories are, you know, going to my grandparents house. And going and eating lettuce from the garden. And eating it at the table with dad.

Eating lettuce as it springs up in early summer, as opposed to buying it from a grocery store in January, carries with it a nostalgia for 'simpler' times when families shared meals together, and the realities of daily life were entrenched in, and in tune with, the cycles of nature. Chloe's account of eating lettuce with her father emphasizes how 'fresh' took on additional symbolic meaning for Green Door patrons in its ability to transform food experiences into positive memories of previous eating encounters. In this sense, freshness

Columbia, who committed to eating only food grown within 100 Miles for a full year. 
captures the aliveness of pre-modern life when both the challenges and pleasures of daily survival were more 'real' (Freidberg, 2009: 4).

Eating seasonally was also attractive to my interviewees because of its capacity to generate anticipation. Despite the prevalence of 24-hour grocery stores selling bundles of imported asparagus and plastic clamshells of strawberries year-round, participants spoke animatedly about their anticipation of asparagus or strawberry season. Specifically, many participants talked about anticipating the seasonal offerings of their local Farmers' Markets: "This summer I was looking forward to it. Like, every Sunday I was like, 'Farmers' Market!' And I would love it." While the delicate texture of new asparagus or the incomparably vibrant taste of a fresh-picked wild Ontario strawberry must be credited in large part for this enthusiasm, an excitement around buying foods just as they become available, potentially only hours after they have been harvested, seemed to be about more than just the quality of the food for these Green Door patrons.

Diana elaborated, for example, on the pleasures of shopping for seasonal produce at a Farmers' Market, "Supporting local people who are trying to make it and not supporting the grocery store that, they have five million chains and they get their produce from California when they could be getting it from Ontario." In this way, Diana and other research participants attached a sense of 'goodness' to their actions of buying 'fresh' food locally thereby reinforcing their perception of self as ethical eaters. The lack of concern for the environmental impacts of corporate business practices and the disregard for the wellbeing of local farmers made shopping from these large corporate food retailers 
unattractive. Highlighting these environmental and social concerns in their accounts of food selection, participants chose fresh seasonal food for its value as a more 'ethical' choice.

However, despite their widely shared disdain for large corporate giants who disregard the 'natural' temporal rhythms of the seasons through what are perceived to be 'unethical' means, Ontario's growing season is short and the participants recognized that they were all required to find alternative food sources when the harvest season dried up. They engaged in a personal dilemma of trying to balance their political views of food consumption with their need to eat fresh food and still maintain a positive sense of self as 'ethical' eaters. As one interviewee said with defeat, "Yeah, during the winter, you're pretty much left to fend with the grocery stores." Buying produce from local farmers all year is a relative impossibility in Ontario's temperate climate, so purchasing foods within this context of seasonal freshness when it is available was described as an important component of participants' personal food practices.

Eating seasonally in the strict sense of only consuming food as it becomes available in a local climate was not seen as a viable option for those I interviewed. While many participants engaged in practices of food preservation, like canning and freezing, to mitigate the 'loss' of fresh seasonal food, all participants also purchased foods that were imported. Jennifer cited 'balance' as a necessary characteristic of an urban food consumption philosophy:

I think it's especially hard this time of year [winter], when good, fresh food is more scarce.... One of the reasons I like this Organics delivery that I get, is that 
they try and do as much local as possible, but then he does, you know, I think he takes the same approach that I do. Like, it's a bit more balanced. So, he'll still throw some bananas in, or we'll get a mango, or something. So that's how I get my local stuff. Through the winter.

Interestingly, imported foods are also perceived as fresh. Following a kind of "it's happy hour somewhere" logic, a number of participants welcomed 'fresh' fruits and vegetables grown, for example, in tropical locations, on the presumption that this food was 'in season' wherever it came from. Again, freshness was conceived in terms of a temporal closeness to the 'natural state' of the food. 'Fresh,' in this sense, could describe any perishable food that appears on grocery store displays in an optimal state of readiness for consumption. Importantly, this definition of 'fresh' took priority, at times, over the need to take a stance against worker exploitation, which interviewees acknowledged to be common practice in many of the source countries from which this food is obtained.

While participants often expressed some regret about making these purchases, they nonetheless wanted to enjoy the benefits this 'fresh' food would afford them as healthy 'eaters.' Popular knowledge supports the thinking that an individual with cold or flu symptoms should have a ripe, juicy orange to boost their levels of vitamin C, despite the impossibility of this food being grown in any season in Canada. For example, Robert spoke about 'eating seasonally' completely outside the context of any traditional links between harvest times and food availability. Instead, he chooses foods depending on what he perceives to be his varying 'needs' at different times of the year. Explaining his consumption of specific natural, nutritionally accessible foods as components of a personal strategy to balance probiotics and $\mathrm{pH}$ levels, Robert said, "In a season I need 
this. In another season, that might be harmful to me." Foods that appear on grocery store displays in an optimal state of ripeness, despite having been imported from great distances, are valued as 'fresh.' According to Robert's personal food ethic, imported fresh 'seasonal' foods are not only enjoyed, but required at risk of harm to him if he fails to eat in this fashion.

\section{Non-Contaminated}

A third value that emerged consistently and clearly in my data reflects this link between food and risk. Though participants valued the variety of 'fresh' foods available to them within our contemporary urban food system, each one articulated their concern that this variety was possible only because of the pervasive use of chemicals in industrial food production. For example, when asked what makes food 'good,' Amelia replied:

Um, to me, it means that there are certain processes that it has not been through....so for fruits and vegetables they do not have pesticides on them, they haven't been subject to being sprayed by chemicals. Um, and when it comes to meat that they haven't been pumped full of antibiotics. So it's more what they haven't been through. What they haven't been subject to.

For Green Door patrons, foods that have been treated with chemical, pesticides, or antibiotics are de-valued because they are no longer 'natural.' Conversely, foods that have not been treated with these materials are 'fresher,' and more valuable; they have not been contaminated, and are therefore 'closer to their natural state.'

When speaking to this point, participants consistently used the term 'organic' to describe these foods, and all participants spoke about efforts to buy 'organic' products. As Amelia claimed, "Most of the food I buy is organic." Christine clearly articulates the 
link between notions of freshness and organic in her description of food at The Green Door: "I know everything is made with fresh ingredients, probably a lot of it is organic, um, and sometimes it's stated, 'yes, this is made from organic.' In this quote, fresh is used as the general qualifier of 'good' food, with 'organic' specifying that this 'goodness' relates directly to the food being un-contaminated by chemicals. This type of 'fresh' is also valued in multiple capacities, as more delicious, healthy, socially conscious, environmentally friendly, and, therefore, 'ethical.'

Recalling his initial skepticism, Jon offered the story of his conversion to organic foods as testimony to their merits:

I had my skepticism dimmed when my mother made me taste test, compare an organic yam, and an inorganic yam. There is no comparison....There is NO comparison. So I ... or, or, um, another really good one is, uh, organic carrots. I mean, organic carrots. Like, uh, when I eat carrots now I can just taste like, I mean not only are they woody and like, a lot of both organic and inorganic carrots are, but like chemical taste! You can actually taste the chemical!

Again, taste is cited as a primary benefit of eating fresh, non-contaminated food; the real, natural taste of the food is contrasted against a woody, chemical alternative. Interestingly, Jon identifies the subjects of his taste test as organic and inorganic. Rather than simply viewing the foods that had been treated with chemicals as natural foods that had perhaps been compromised to some degree, Jon seems to indicate that these foods can no longer be perceived as living organisms (the literal definition of the term) but rather as a synthetic chemical variation. This type of judgment is significant in that it essentially removes the value of 'inorganic' food as a source of life-energy. This process of elimination described by Jon reflects the widespread consensus among my interviewees 
that organic food is clean, pure, natural fuel for the body. For a living, breathing, renewing body, organic food is the optimal choice. As such, to be a healthy, ethical eater means eating organic.

Further, the Green Door patrons I spoke with believe spraying crops with pesticides or an indiscriminate/overuse of antibiotics on livestock (both common practices in big agribusiness) have important environmental and social impacts. As such, organic food is also perceived as valuable in that it has broader environmental and social benefits for the world in which they live. Rachel remarked:

I think it's more importantly, obviously the environmental things. They're not being treated with pesticides. Um, and just sustainable agricultural practices, knowing how the people um, I guess have been, not just taking care of the land, but also the kinds of seeds they're using or the kinds of things they're growing. Um, just again, I guess, to maintain some kind of diversity. And noncommercially-streamed seeds, kind of thing.

While Rachel spoke to the environmental impacts of chemical-use such as soil depletion and loss of diversity, others noted the effects of these toxins on those who work the land. Because one interviewee had spent time in South America living and working with coffee growers whose health was negatively impacted by chemicals they were forced to use on their crops, she makes it a priority to choose 'organic' when she is purchasing this item:

The motivation is mostly the chemicals, like the uh, pesticides and stuff that are on non-organic. Um, and also the growing practices. We do make a point of...drinking fair-trade, organic coffee. Um, so that's the one thing that we're pretty strict about.

Interestingly, certain non-organic foods items were perceived to have greater negative effects than others. While discussions of buying local and eating seasonally often related to fruits and vegetables, many participants specified that animal products 
most needed to be organic. When asked which organic foods she purchases, Julie answered:

I don't really buy organic vegetables, unless, no I don't actually. I'm more on the organic meat. There's something, something I find really disturbing about ingesting hormones and steroids and that sort of thing that I'm getting through the meat. And I'm sure that I'm ingesting ... now I'm depressed ... (laughing) I'm sure I'm ingesting chemicals with vegetables, but I don't ... I'm not as conscious about it when it comes to vegetables. In terms of buying organic. I buy organic meat. And organic milk before I buy organic vegetables.

Asked the same question, Ann quickly responded, "Definitely milk. Milk for sure. Meat if I can afford it. Or if it's on sale. Sometimes it is... Um, eggs." Later in the interview, Ann explained that she stopped drinking 'regular' milk because she was told it might be contributing to her difficulties getting pregnant:

I stopped drinking regular milk, and switched to organic. So, like, there was no hormones. No preservatives. No MSG. Which is linked, you know, with brain function, you know. So I just kind of realized then, like oh my god, I'm poisoning myself.

Having conceived her daughter shortly after making this switch, Ann was adamant that 'regular' milk is not only less healthy, but toxic and detrimental to 'natural' processes of reproduction. Similarly, while tearing up at the end of the interview Helen admitted, "I worry about contamination. I worry, quite a bit about chemicals given to fowl and cattle...I worry about the contamination, chemicals, hormones, uh, also, also, uh, you know when you hear that people are getting these terrible diseases from eating certain, like it was beef for awhile."

Avoiding animal products that have been produced in factory farms was also cited by these Green Door patrons as an important ethical benefit of buying organic. Factory 
farming practices include heavy use of antibiotics to keep animals 'healthy' in overcrowded, poorly lit, stress-inducing warehouses that provide optimal conditions for widespread transmission of infectious disease. Non-organic animal products are not fresh because the conditions in which they are produced, including a diet of antibiotic laced corn and extremely limited access to natural light and fresh air, creates a disconcerting disconnect between the product and the 'natural' conditions of a traditional farm. Thus, for example, Rachel insists on organic yogurt because of her ethical objections to producing animal products this way, as well as her personal concern to avoid ingesting high levels of antibiotics:

Like, I'll eat yogurt, but it tends to be organic yogurt. 'Cause I'm still not a huge fan of um, obviously the idea of factory farming and the way milk is produced now-a-days. And so if I can be relatively sure that it's free of antibiotics I'll eat the yogurt.

Each interviewee made it clear that the prevalence of food that is treated with 'unnatural' chemicals was a cause of major concern for them. When asked how she would eat if she did more of the grocery shopping, Helen said, "I would have to buy organic, because that, there's too much of a worry about the chemicals, right?"

To address this anxiety, my interviewees participated in organic food consumption in a variety of capacities. Some made special trips to specific food retailers based on their selection of organic meats, and a number of individuals had organic food boxes delivered to their homes. One participant, Elle, also spent time in Washington as a lobbyist for the Organic Consumers Association. In these ways, they made consistent efforts to align their need for personal health with their perception of self as ethical eaters 
who supported positive environmental food production through the consumption of organic, fresh products.

Importantly, participants acknowledged the controversial and contested nature of the organic label. Tina asserted: "I'm also very critical about how those labels get used." Jon also noted his past reservations about adopting an organic food ethic:

Yeah, it's a charged word. It's a bit of an annoying concept. I was sort of like, anti-organic for a while, because you know, I thought it was expensive, and it's marked up. And I thought, you know, who really knows if it's organic or not. I mean, to me, my understanding is that it's, it doesn't include chemicals. Or uh, yeah, you know, unnatural chemicals.

Jon's comment is significant because it speaks to many of my interviewees' attempts to consume natural, chemical-free foods while similarly reflecting the fact that, ultimately, they have limited power to monitor their food consumption under an industrial food production market. Despite some telltale differences in taste or appearance, the processes and practices of industrial food production are largely invisible to consumers.

Consequently, Jennifer admitted: "That kind of worries me, that people really, you know, it's amazing what people will put in their body without even realizing it." Ann, a dedicated consumer of organic foods, also revealed that she really does not understand the requirements of organic in terms of farming practices, but rather equates organic with simple, natural foods: "Oatmeal is about as basic as you get. It's probably all organic really. Maybe not. I don't know much about it really." This experience of not knowing, common amongst those I interviewed, both adds to individuals' anxieties and increases the value of an organic label that clearly signals safety. 
While interviewees expressed concern that this label has been adopted by large corporations whose use of the term is more a marketing strategy than an indicator of adherence to regulation, many said they were ultimately willing to trust these claims (and pay higher prices for products) because the risks of not doing so were unacceptable. Thus, a number of interviewees said they were encouraged by the launch of an Organics line in Walmart, or the expansion of the Organics section in Loblaws because it made their efforts to buy non-contaminated foods more accessible. For example, Julie asserted, "Loblaws is really good now, because they have all of the Free-From meat. So it's like, sectioned, so in the meat section you can buy chicken, or you can buy chicken that's free from steroids, and antibiotics and stuff like that... So with those options, l'll always buy better."

My research participants' attempts to avoid chemically contaminated food reflect their broader desire to consume food that is 'as close to its natural state as possible.' The value that they assign to fresh organic food is also related to both their personal experiences (in terms of taste and mitigating risk) and their broader efforts to engage in their perception of ethical food consumption. The organic movement of the 1960's has helped to create a widespread awareness of the dangers of chemical-use in farming practices and the use of this term has come to dominate contemporary food discourse (Guthman, 2003). However, as I will discuss in the next section, the use of chemicals in processes of food production is not the only way these participants saw food as being contaminated within our contemporary food system. 


\section{Non-Packaged/Non-Processed}

Another cornerstone of 'good' food consumption for these Green Door patrons was the rejection of packaged or processed foods. Perhaps the most widely shared consensus across interviewees' responses was the belief that packaged or processed foods are to be avoided. Stabilized with high-fructose corn sugar, salt, or chemical preservatives, and wrapped, boxed, or canned within inedible, synthetic materials, these foods are decidedly perceived as not fresh. Participants did not view packaged foods as fresh because the processes the foods are put through and the packaging they are presented in reflects an often complete disembeddedness from the ingredient's 'natural state.'

Amelia's response to my question what makes food 'good,' illustrates participants' perceptions of the link between non-packaged foods and freshness: "I guess freshness. Ahhh.... yeah, it being fresh and real. Um, no matter what it is, yeah, I think that's probably my number one criteria." When I asked her to clarify what she meant by 'real', she said:

Yeah, well I avoid um.... well I think it goes back to the avoiding the processed. Avoiding anything that has been sitting ...Processed for me means anything that is packaged, pre-packaged.... And [food that's non-processed] is fresher.

Helen also spoke to a personal commitment to 'real,' as opposed to packaged, foods: "Nothing from, nothing um, prepared. Absolutely nothing. Like, everything is made from scratch and it's made from real ingredients." Similarly, Julie asserted that 'real' food is the homemade Italian food her grandparents prepare:

So, for example, um what makes Italian food real to me? What makes Italian food good is, like to me it's the sauce. So, like sauce out of a jar? I cannot. I 
cannot. I can't. I can't eat it. It tastes processed. It tastes like...it tastes like sugar!

The consistency with which the concept of 'real' is employed by the interviewees as a counterpoint to 'packaged' in my data is striking. Although the term is problematic in its ambiguity, the similar words, phrases and examples used by them in describing 'real' food give it substance. As such, I argue that participants' desire for 'real' food reflects the link I propose between freshness and food that is in its 'natural state.' Julie illustrates this point:

I would only buy a whole chicken, rather than chicken breasts in a package, I would buy the whole chicken...Because, um, the idea is that it hasn't been as processed. Right? Yes, it has, it has been plucked and it has been packaged, but you're not getting, you know, a package with like, 12 legs of a chicken. You're getting the whole chicken and then you deal with that. And then I would use the bones to make stock. And tried to use it more like that.

Although the 'processing' of dividing and packaging chicken into parts is relatively minimal, compared, for instance, with the drying, refining, cooking, toasting, stabilizing, individual wrapping, and packing that produces a box of granola bars, it nonetheless disconnects the food product from its 'natural state.' A whole chicken is more valuable because it is recognizable in its fresh, natural form and, as a result, is perceived to be 'real' food.

The irony of this development is that the original impetus for packaging food was to increase the 'value' of perishable, or labour intensive edibles. Selling packages of chicken breasts was designed to offer consumers the opportunity to enjoy only the highest quality cut of meat; boxes of dry sweetened cereals provided consumers with a quick, convenient way to 'start the day off right;' and canning food was the solution to 
enjoying 'fresh' foods all year long in any circumstances (Freidberg, 2009). Now, however, as my data indicates, these innovations have created products that represent the antithesis of 'good' food. Thus, instead of enhancing the value of the product, with added convenience or stability, packaging is perceived to diminish the value of fresh food. Alana offers an explanation as to why packaged or prepared foods should be avoided:

I guess for me it's about being connected to the ingredients. And as soon as you're bringing a prepared product, you don't really know what the ingredients are any more. You've lost sight of what you're putting together. And I just sort of feel like someone else is controlling that for you? ...... in the most basic way, like, something that you understand. At the very least.

Many research participants spoke about their desire to reconnect with whole, real ingredients because they fear that packaged food contains hidden danger. Fresh, as opposed to packaged, allows the individual to maintain control over the processes their food undergoes before it is eaten. Ann articulates this perception in her discussion of fresh versus packaged food:

I would say, in general, I like to eat things that are fresh. And not from a package. Um, I'm not a vegetarian or anything like that, but, if I do eat meat, I tend to gravitate towards the organic meats. Um, basically, I just don't eat anything out of a package. Other than, you know, maybe pasta, or, you know, cereal, I guess. But, you know, generally speaking, I peel it, slice it, dice it, myself.

"Peeling, slicing, and dicing" whole, natural ingredients may take time and energy, but this action also ensures that food is safe. It is still fresh and, hence, non-contaminated and healthy for the food consumer to eat.

Interestingly, fresh food does not need to be low in fat or sugar (ingredients that are commonly vilified in popular media as dangerous or at the very least, unbecoming). 
According to my interviewees, food just needs to be prepared from real, recognizable ingredients. Contrasting her current personal food ethic with one that used to include frequent trips to convenience stores for Maltesers and potato chips, Diana said:

I've decided my junk food is going to be baking. Like, if I'm going to eat junk food, then I have to bake it. So that way, you know what's in it. Like, you put whatever's in it, there aren't preservatives and enrichments that you don't know what it is, so it's kind of, like, it's still junk food, it's still not really good for you, but at least you know exactly what's in it. So, sugar, flour, cocoa, it's not anything weird.

This sense of feeling more safe and in control was only one of the benefits cited by participants of eating fresh, non-packaged, non-processed foods. For example, Robert, a self-described 'live" ${ }^{6}$ food eater, represents a faction of eaters who completely reject any type of food processing. Robert argues that live, 'bio-available food' is nutritionally more accessible:

Any food that has not gone over a hundred and five degrees is still alive in that it contains all the biomechanical elements that it needs to be digested. And that's why people who eat raw food tend to be more energetic rather than less. Because it actually wakes them up - when I eat a meal, it wakes me up. And most, when people eat a cooked meal, it shuts them down. It makes them go to sleep. Live food is something that is actively living itself. It hasn't been pasteurized, it hasn't been cooked, it hasn't been boiled, it hasn't been distilled, it hasn't been processed...

Fresh non-processed food is again valued as a source of clean, live energy. Thus, Robert views the consumption of fresh, non-packaged foods as vital to enjoying a high quality of life, arguing that eating processed foods depletes the body's energy. He offered his

\footnotetext{
${ }^{6}$ A 'live' or 'raw' diet is composed of unprocessed plant foods that have not been heated above $40^{\circ} \mathrm{C}$ $\left(104^{\circ} \mathrm{F}\right)$. Those who adopt this diet believe that foods cooked above this temperature have lost much of their nutritional value and are less healthy or even harmful to the body. Raw or living foods have natural enzymes, which are critical in building proteins and renewing the body. Heating these foods kills the
} 
experience at a raw-vegan potluck as testament to the positive, energizing effects on the body of food that has not undergone any processing: "Nobody was in a food coma ... Everybody was alert and active. And they were more alert and active after the meal, rather than less." In a similar fashion, speaking again about her time on Kandahar Air Field in Afghanistan, Erin reminisced about the times she lived off the main base and was able to try the local fruits: "Being able to eat fresh fruit is better than canned fruit. It makes a big difference." Erin commented that this 'difference' was related to levels of morale among soldiers. Because they ate 'from scratch,' they were able to maintain the level of fresh required to keep their bodies healthy and their morale intact.

While participants agreed that cooking from scratch with whole ingredients is the best way to eat, they acknowledged the difficulties of defining this value. Levels of processing (and the associated value, or lack of value, in that food) was understood within a continuum of 'freshness:'

So, I mean technically, when you buy organic bread, technically that's processed, so that I will eat. When I think processed I think big box, middle of the grocery store kind of stuff. So anything that is pre-packaged in a box and is going to sit on your shelf for a year or two. (Giggling) For the most part, I'm not going to put it in my body. Because it's a little scary to me; like what do they put in to make it last that long? Not refrigerated, on a shelf.

Katherine later qualified her claim that she does not eat processed foods:

So if I do buy pre-packaged food, it's from there [Market Organics grocery store]. And it's like, it has an expiry date that's usually a day or two later, and I know that they probably made it in the kitchen in the back. So it's good. But even then I try not to do that very often either.

natural enzymes, and can leave toxins behind. Robert preferred the term 'live' eater over 'raw' because he feels it better captures the natural vitality of uncooked food. 
While participants shared the view that packaged, processed foods are undesirable and even dangerous, they all acknowledged and accepted that their consumption practices included items that fell somewhere on this continuum of 'freshness' ranging from 'processed' to 'non-processed' foods. Participants did not generally seem concerned with how much their food had been 'processed' (embracing, for example, the multi-phasic process of transforming a sheaf of wheat into a loaf of bread), but rather by which means processing had occurred. The preceding quote relates to a final dimension of the freshness that emerged from my data. While food that was processed and packaged within the mechanized structures of our industrialized food system was de-valued as alienated from its 'natural state,' participants indicated that food could be processed in a particular way that not only preserved, but could enhance its value as fresh, good food.

\section{Human Handled}

Participants' distaste for mass-produced, packaged food was countered by their shared, and equally strong, appreciation for food that had been prepared by people. Recognizing that food is almost always put through some sort of 'process' when it is prepared for consumption, they state that human handling does not remove the 'freshness' of the food, but rather highlights the 'natural' quality of the ingredients. Establishing personal connections with the people who produce or prepare one's food, and becoming personally involved in processes of food production and processing, were articulated as viable strategies for getting as close as possible to the 'natural state' of one's food. The 
energy that people put into food preparation enhances the freshness, and therefore value, of the food.

For example, when I asked Chloe to clarify what she meant by 'high quality' food, she said:

It would be something that makes it.... Sorry this is hard ... uh ... yeah, something that is, something people have taken care to choose the ingredients and that it's not coming from, you know, a package, say...I guess, though whole ingredients do come from packages. But yeah, I would say that....I tend to go to places that selfdescribe as putting thought into the food that they are preparing.

Amelia also articulated this sentiment in her description of fresh 'processed' food in her discussion of why she ate at the Green Door:

And it's fresher... and it's not...I guess, food that is prepared on site, with ingredients that they have. Versus, an establishment that, uh, things are pre-made and they're just throwing it together.

These types of comments reflect the widely shared belief among the interviewees that thoughtful, caring cooks (or farmers, bakers, or butchers) who prepare their food 'fresh to order' produce 'good' food. For instance, Katherine recalled: "When I went to India I ate so well, and it was so much easier to eat well, right? Because everything is sort of there, and it's available, and they're making it on the spot." In this way, speaking specifically of the roadside cooking stalls she ate from in India, Katherine argued that the food was healthier and generally better because she could watch her meals being 'made fresh' from local ingredients by a single person who carried the food through the entire process from whole ingredients to a spicy, flavorful, steaming-hot curry.

Research participants agreed that the successful preparation of any 'whole' ingredients relies on thoughtful, skillful preparation. One interviewee, who raved about 
her husband's mastery in the kitchen throughout our interview, argued that 'good' food is food that has been handled with skilled attention:

And um, we, we eat lots of organ meats. We do. We eat livers. We love livers. They taste good, 'cause they're prepared well. It's all about preparation, right? I mean, everything can taste good. But you do have to know how to prepare it.

When consuming less popular cuts of meat such as liver, interviewees see skilled handling as a necessity. In a follow-up discussion about her husband's skill, Helen made an interesting link between preparation and freshness: "And um, uh, so he will get either pork or veal, but he makes pork taste like veal, so ... it doesn't really matter." Thus, Helen claims that her husband's preparation not only brings out the good qualities of the pork, but transforms it in to an even younger, more tender, and 'fresher' product. As a result, a temporal freshness can also be achieved through the skill of the person preparing the food.

Julie also speaks to the possibility of producing freshness through personal food preparation. Rooting through her cupboards and holding up a vintage coke bottle filled with tomato sauce, Julie illustrates the value of food that has been prepared by hand: "So for example...This is tomatoes. And this is made, my grandparents still crush their own tomatoes. This happens in the fall. It's called 'Making Tomatoes'." 'Making Tomatoes,' as Julie describes it, is a multi-phase process that includes buying Roma tomatoes, laying them out in their garage, letting the tomatoes ripen, de-seeding, skinning, and crushing them with a home grinder, seasoning with various herbs, then sterilizing, bottling, and sealing them. Making tomatoes is a decidedly long and intensive process, but the end product, used as a staple ingredient for her and her family all year 
long, is viewed to be equally as natural, or fresh, as eating the original 'whole' tomato. Julie expressed an awareness of this dynamic when she noted, "It's called 'Making Tomatoes,' even though it's making sauce."

Many interviewees spoke about their personal efforts to become involved in traditional practices of food processing, preservation, and preparation. They argued that getting personally involved in food production, and acquainted with the offerings of each season, had a number of benefits. For example, Tina spoke about re-claiming the 'process' of her food from planting seeds to consumption:

My partner started a vegetable farm this summer, so that was a very intense relationship with food. And just eating whatever we grew, and that kind of thing, and then trying to preserve and store stuff for the winter.

Tina expressed great pleasure in learning about the realities of farming and familiarizing herself with what can be grown, and how, and when it is ready for harvest. Putting this amount of thought and care into the processing of her food, and getting 'as close to its natural state as possible,' afforded her a deep appreciation for the food she ate. From her perspective, the 'freshness' of this food was not only defined in terms of its local sourcing, seasonal consumption, organic certification, or lack of packaging, but also in terms of the freshness of the experience of running a farm for the first time.

While an increased engagement with traditional practices such as canning was at times framed as a hobby, or a novel dalliance with a romanticized rural past, this trend also reflects the interviewees' general mistrust of the larger systems of production that erase the limitations of seasonal food consumption. They saw self existing within a food production system that, as Jennifer put it, "is not really looking out for us." However, 
rather than attempt to personally produce one's own food like Tina, most participants relied on alternative food retailing sources where they could develop personal relationships with their food producers.

Many participants used Farmers' Markets as an opportunity not only to buy local, seasonal foods, but also to get to know the people who were growing this food and learn how those growers approached the food production process. For example, when I asked Amelia how she negotiated her concerns about animal cruelty with a gluten allergy that, from her perspective, made it essential for her to consume meat, she replied:

I have gone to the local farmers' markets, where they sell their products, and have spoken with them directly about how they handle their animals. And um, they seem, to genuinely care for them.

Amelia says that she spends much more money than the average person on food because she insists on buying organic meat from people who care. Another interviewee described speaking with farmers as less an investigative procedure than as an enjoyable practice of connecting the dots between food and source: "I love, well I love that you're getting all your produce there, and you feel like you're getting, you're seeing the farmers and interacting with them." Participants felt an increased 'closeness to the natural state' of their food through connection with its producers. Diana continued:

And even stuff that isn't healthy that I would get there, I would just feel better about buying it there. Like, the girl with the ice cream! When I would get ice cream, I'd get the Goat's Cheese Ice Cream, and it makes you feel good when it's like, her company, her little face is on it, and you see her, and you know who you're supporting. And that's making a good product, that has like, 5 good ingredients.

Diana also spoke of taking pleasure in being recognized by merchants who would follow up on how she had prepared the squash or carton of peaches she had bought from them 
the week prior. These quick, unexpected sparks of conversation were experienced as fresh moments in her day and increased the value of her food as a social practice shared with her food producers.

Robert argues that we enjoy a better relationship with our food when we reestablish relationship with the person that produces it:

When the Farmers Markets were open: We knew the name of the guy who grew our grains. We had a personal relationship with the guy who produced our produce, you know. We hung out, you know, we would go out for tea with the guy who, you know, grew our arugula, and you know, this is wonderful! Um, the person who produced our worm compost is a close personal friend.... Reestablishing that relationship not just with the food, but with the person that produces that food.

This network of personal connections was highly valued by Robert. Similar to Diana, he feels that such connections served a social as well as a supply function that enhanced his quality of life. Other participants echoed this sentiment through statements of the value their personal connections with food producers held for them because opportunities to forge these relationships have largely been eliminated from our contemporary food system. Speaking of her participation in a $\mathrm{CSA}^{7}$, Jennifer exclaimed, "And it was incredible for the period of time that it lasted, to know exactly who grew my food. I mean, that's incredible."

Foods that have been 'processed' by people were not only seen as superior in taste, having been handled with thought and care to bring out and emphasize the natural

\footnotetext{
${ }^{7}$ Community Supported Agriculture (CSA) is an alternative model of food consumption. A CSA consists of a community of individuals who pledge support to a farming operation where the growers and consumers share the risks and benefits of food production. This mutually beneficial commitment provides consumers with a direct link between the production and consumption of food. CSAs usually consist of a
} 
value of ingredients, but they also afforded these Green Door patrons with a sense of safety and community. Whether they increased their personal involvement in processes of food production and processing, or established personal connections with the people who did this work for them, the participants in my research study agreed that people's active participation in the production of their food makes that food 'fresh.'

\section{Negotiating Values}

While all of my research participants spoke to local, seasonal, non-contaminated, nonprocessed/non-packaged, and human handled values as important components of their personal food ethics, none felt able to attend to all of these values in all of their eating choices. These individuals had to navigate between values, often making concessions in one area to fulfill another food value in their attempt to create an overall diet that was 'good enough' in terms of their perceived need for food to be fresh. Katherine summarizes the difficulties of negotiating the component values of the fresh food ethic:

I really do subscribe to the idea that .... Ideally [food should be] locally sourced and organic. And again, nutrient dense. For me, those are quality foods. Because if they have those things, typically they taste really good. The difficulty with that though I think, is, number one, there is a lot of contradictory information out there. So as much research as you do, it's hard to decide which foods to buy. Cost is an issue sometimes. And then, what do you do when you're faced with the situation of: I can buy this local, but it's not organic. Or I can buy organic, but it's not local. And personally, I would probably choose organic, because I know. As much as I would hate to do it. Unless it's from a farm that I actually know in the area and I actually know the people that run it. Because just because the farm is, you know, 100KM away, doesn't mean they don't use pesticides in there. You know what I mean? It's tough some times to make the right choices. 
The challenges of making the 'right' choices emerged as one of the most significant dynamics within my data. In this section I will outline the core challenges my participants identified in their interviews and explore the implications of these challenges by making links to the broader social context in which food production occurs. I argue that individuals engage with the 'fresh food ethic' as a reflexive project of the self, adopting these values in varying configurations and combinations to create a highly personal food practice. I will show that the 'fresh food ethic' reflects an 'omnivorous' approach to food knowledge wherein individuals pick and choose 'knowledge' from a limitless spectrum of food wisdom. Rather than looking to a prescribed, expert-endorsed food regimen, individuals work reflexively with these bodies of food knowledge to construct a personally negotiated approach to food consumption that promises custom-fit optimal wellbeing. I conclude that despite the markedly personal nature of an individual's food identity, all of my interviewees' personal food practices operate within a broad, and shared 'fresh food ethic.'

\section{Consumption Challenges}

My data indicates that making the 'right' choices is complicated by three primary challenges: financial limitations, social factors, and contradictory values. The financial cost of adhering to these values was cited as a challenge for the vast majority of my participants. For example, in explaining what she considers when choosing what to eat, Tina said, "more about ... price, like you know ... You know, grad students. Grad student plus farmer is not, like, the (Laughing) ... the best." Describing a personal food ethic that 
includes buying organic meat, Amelia agreed, "Obviously it's more expensive, so it's financially challenging." Chloe also cited price as a prohibitive factor in food selection. Calculating the cost of buying local and non-packaged foods, this interviewee concluded: "organic stuff tends to be that much more expensive." She said: "I try and push my husband when we're buying meat or dairy or eggs for him that those things should be organic or at least hormone free as much as possible." However, she finds it difficult to persuade her husband to buy organic because of the higher cost.

Participants also noted the impact of social factors on their food choice. Thus, when I asked Amelia what she considered to be the biggest challenge of eating according to her personal food ethic, she replied:

The biggest challenge is social. Socially is definitely the biggest challenge. When I go out with friends, or any one at all, there's always this anxiety over, will I be able to eat where we're going? Or if I can eat something will it just be a salad so I'll be starving for the rest of the night?

While Amelia can have a 'fresh' salad most places, she finds it difficult to get enough of what she needs. Ann also argued that the challenge of integrating herself into social circumstances was considerably greater than any internal challenge of choosing the right things. She pointed out that even salads are not a 'good' choice when going out with friends, because restaurants use salad dressing 'out of a bottle:'

Because if I'm out, even in restaurants, depending on where you go, I mean, there are no healthy choices sometimes. Like some of the sports bars in Ottawa. You have zero choice on the menu. There is literally nothing good for you on the menu. (Laughing). Maybe a salad, but you know, it usually comes with bacon, and some kind of garlic, Jack Daniels dressing or something right from the bottle.

While these passages point to important structural challenges (including a lack of 'fresh' options on mainstream menus), my research participants consistently framed their 
concerns as being social in nature. They often insisted that there are foods they can eat and places they can go to dine, but that they feel guilty complicating others' social plans with a need to accommodate their dietary choices and often feel like these dietary concerns cause them to 'stick out' rather than 'fit in.'

Commenting on how she feels about being vegetarian within her husband's meateating family, Renee admitted:

I don't really want to make a spectacle of it. I don't want to say, no, I'm not having, like any of what you're eating. I find that embarrassing. Personally. I guess I worry about what other people think. That's part of the problem.

The feeling that an individual's personal food ethic cannot easily be fulfilled in a social context was widespread in my interviewees' accounts and articulated as a significant challenge in maintaining their dietary goals of eating fresh.

The Green Door patrons I interviewed also noted that the values themselves were often incompatible with maintaining 'fresh' eating habits. For example, one interviewee explained that her decision to buy more local foods has resulted in her buying less organic: "Organic used to be more important and has become less important to me ...because there aren't very many organic, local producers." Elle also struggled with the difficulty of integrating the local value within her raw vegan ethic stating: "Obviously local is better if that's possible. But again, being a raw vegan, you do eat a lot of tropical fruits, or whatever, so you have to get around that as well." For many of the interviewees navigating these values was perceived as an evolving process that requires continuous reconsideration depending upon one's social or environmental context. Thus, for example, although Elle identified strongly as a raw vegan eater who is committed to consuming 
completely non-processed foods, she admitted that she is required to prioritize particular values:

I realized recently... to me eating organic is almost more important than eating raw, or, I mean, if the situation would come up, probably even vegan. To me, [organic] is more important.

One of the main challenges cited by participants was finding produce that was both non-contaminated and at an optimal state of ripeness. Renee cited the organic value as her top priority:

Primarily it's the organic versus non-organic. Like, I don't make any great effort to trace where my food comes from in particular. But, um, I tend to trust in the fact that if it's organic it is produced ethically and the people are treated well who make it. So that's the main thing.

However, this seemingly clear-cut value was complicated for her by a competing priority:

Like, sometimes you go to the supermarket and the organic apples just look horrible. Like, they're soft and bruised and you know, as much as I want to buy organic, I also want quality food.

In this way, Renee described a decision-making process in food selection - experienced by all of my research participants - that was not bounded within the realm of ideology, but rather navigated 'in the field' of choices available to them:

We make a beeline for the organic section, see what's there, that we can afford and looks good. We'll pick it up, and then, whatever's not there we'll get in the non-organic.

More than revealing what could simply be dismissed as evidence of hypocrisy, this decision-making process highlights a tension in food values that characterized my research participants' narratives. Although choosing between a bruised organic apple and a shiny, chemically treated apple may be perceived to be a relatively innocuous "tension" in and of itself, I argue that this decision (compounded by hundreds of other micro-choices that 
individuals make each day) reflects an important dynamic in our contemporary Canadian foodscape. Specifically, those Canadians who seek the types of choices being made by the people I interviewed enter a multi-faceted terrain in which food becomes part of their perception of self as ethical eaters and healthy members of their society.

\section{Making Informed Choices to Navigate Risks}

Scholars have shown that cultural meanings of food are now "dominated by perceptions of risk and anxiety" (Ashley et al. 187). They see that food is a particularly significant social object because it is susceptible to contamination and, once ingested, becomes risky at the most intimate level. Widespread media coverage on the links between diet and disease, including reports that mark the rise in diabetes, heart disease, and a growing "obesity epidemic," highlight the risks of making poor dietary choices (Lupton \& Chapman, 1995). Beyond concerns about chemical contamination from food additives, artificial flavorings, and pesticide-use, there have been recent food scares around E. Coli and BSE (mad cow disease), which demonstrate the failure of our industrialized food system to produce safe, healthy food. This knowledge requires individuals to question the systems they have entrusted to produce food and be vigilant in how they navigate through those food production systems. In the case of the apple, for example, Renee is not simply making a choice about what she wants to eat, but is also weighing the short and long-term risks of toxicity on her health and the environment. Given such instances reported in my data, I argue that the individuals I spoke with engage in a process of 
reflexive decision-making that allows them to navigate these risks in a way that affects their perception of self as healthy, ethical eaters.

Interviewees indicated that they look to a wide variety of information sources to make informed decisions about health and safety. All of my interviewees spoke about doing 'research' that informed them on the values of the fresh food ethic. Robert asserted, "I take in information about food constantly ... Mostly on the Internet...Also, I love the public library." Amelia reported similar activities: "I do a lot of reading. I know so much about nutrition and food from my own reading books, and on the internet." To offer some specific examples that guided my understanding of interviewees' 'research,' Helen "highly recommended" a book called Sugar Blues, which posits that white sugar is "modern day poison" and outlines the dangers - most notably depletion of immune function - of consuming foods that have been refined in processes of industrialization. Amelia summarized the central thesis of The Paleolithic Diet, which recommends returning to a diet comprised of the 'natural' foods that were eaten by our hunter-gatherer ancestors as a means of strategizing against disease. Gardening magazines and cookbooks were also cited as resources used by the research participants to re-connect with the human handling of their food. Chloe revealed that these resources provide ideas on how to eat more seasonally:

I also have a huge list of book marks in my box of recipes that I want to try at some point and a giant stack of cookbooks ... And some of my cookbooks are more summer cookbooks, some are more winter so, sometimes I'll do cookbooks, sometimes I'll go through Vegetarian Times. I'll go through all the old ones from that particular month and look for recipes. 
Jennifer said that she follows "a lot of food blogs" to get fresh recipe ideas and recommendations on where to go to enjoy meals made from locally sourced, organic ingredients. In addition to reading and searching the Internet, research participants spoke about watching documentaries to familiarize themselves with any potential threats that result from contemporary industrialized food production. Katherine, who has been certified in nutrition and weight loss management, said that she is always "expanding [her] scope of food knowledge and doing a lot of [her] own research:"

I read...um, some Jillian Michaels, which I felt kind of silly buying.... big TV personality. But it's actually quite useful. There's one [book] called The Hormone Diet, which I read.... All of the Michael Pollen ones... And you've probably seen Food Inc. Um, so I've seen those types of documentaries as well.

Food Inc, an award-winning documentary film that highlights the horrors of factory farming and the wide spectrum of problems that emerge from relying as heavily as we do on genetically modified, chemically treated, mono-culture cash crops, transmitted 'fresh' values to a wide mainstream audience. Similarly, the authors Katherine cites in this passage are popular advocates of 'fresh' values. Other participants discussed Michael Pollen, author of The Omnivore's Dilemma (2006) and In Defense of Food: an eater's manifesto (2008), who has become a seminal figure for a huge mainstream audience for his critique of the 'industrialization of eating.' Arguing that our contemporary urban foodscape has devastating consequences in terms of our ecological and individual health, Pollen explores the dangers of chemical contamination and outlines the merits of locallybased food systems. Jillian Michaels, fitness guru on American reality TV show The Biggest Loser and author of Master Your Metabolism, was also noted. She explains how 
different foods work within the body on a micro-scale and outlines a diet strategy that uses "natural" foods to balance hormones. Michaels also provides "quick and easy" recipes that encourage the reader to re-connect with the human handling of whole ingredients.

Importantly, participants indicated that this research was not simply an intellectual exercise for them or the product of idle curiosity. All were adamant that the information they collected from these sources was critical to their wellbeing and was translated into action. As such, Robert passionately described his commitment to this research when he said:

I know what goes in my food. Um, and yeah, I'm kind of putting a big ' $\mathrm{k}$ ', a capital ' $k$ ' on the word 'know.' Because it is about that certainty, that knowledge of what the food is (and how it was produced).

Robert also noted that this quest for Knowledge is only relevant if it is accompanied by a willingness to change one's behaviour: "I will always change my opinion based on new information." In a similar fashion, Katherine offered an example of how her choices have shifted since she began her education:

Now I'm much more aware of the composition of my meals. So, the macronutrients. And then also the actual micro-nutrients that I'm getting. Like am I getting enough anti-oxidants? Which anti-oxidants do what? So, just the general effects of food on your body, and the kind of, "food is your medicine."

Making reference to the same documentary film cited by Katherine, Diana indicated that her research has shaped her behaviour:

Like when you read, when you watch, like Food Inc.... And you hear about how terribly the cows are treated ...My parents have a thing with a food co-op, where they buy meat responsibly, so now we get it through them. 
Specifically, research participants indicated that their personal food practices were shaped by concerted attempts to manifest ethics (which they had developed through research) into action. Tina asserted:

I try and put a lot of emphasis on tying my ideas to my actions. And so if I think a particular way, I spend a lot of time trying to actually figure out how to do that in my own personal actions.

This integrity around food consumption is linked closely to notions of discipline, especially, in the decision-making process involved in food choice. Thus, Tina added:

Trying to be really disciplined with my food speaks to the fact that I try and be a really disciplined person about my like, values and my ethics, and implementing them into my daily life. Um .... I guess if you were being negative you would say, like, militant. (Laughing). I'm very, probably ....... like, militant about my politics in terms of like, how they inform my daily actions and behaviours and relationships.

Making these important links between values, ethics, action, and identity, Tina speaks to the importance of maintaining personal integrity in her food practices. Interestingly, a number of interviewees used the term 'militant' to describe their attempts to maintain integrity between food values and food practices.

I've gotten to be more militant about it. Ahh, I wouldn't say militant. More particular. In some ways it's a challenge. .... I'm also very conscious of where food is coming from so, you know.... I'm really careful about where it's coming from and what season things are in.

Thus, while Chloe quickly backed away from a militaristic language, she clearly articulated the view that constructing a personal food practice requires vigilance. 'Good choices' do not just happen; they are made by informed actors. Individuals engage in a reflexive process of information gathering and evolving action, relying on personal discipline to maintain integrity. Importantly, the participants in my study indicated that 
their allegiance to particular food practices (and the discipline this allegiance required) was self-imposed rather than reflecting compliance to an external authority. While participants acknowledged that broader structural conditions play a role in shaping their beliefs and priorities, they spoke with the belief that they were free to explore an essentially limitless range of information sources to construct a personal food practice that was custom-fit to meet their personal needs.

\section{Personal Food Practices: An Omnivorous Approach}

The final, and perhaps most significant, piece of this discussion is the highly personal nature of the food practices my participants described. While the fact that all of my participants described a practice of systematic food research is significant in and of itself, I argue that the integration of this research into completely individualized personal food practices is particularly interesting. Rather than attaching self to a single dogma (e.g. religiously following The 100 Mile Diet, or Weight Watchers or citing the PETA mandate as a personal mantra), each of my interviewees integrated various bodies of knowledge to construct an individualized food practice. Based on their accounts of this process, I argue that this construction can be conceptualized as an omnivorous approach to food knowledge. That is, individuals demonstrate an appreciation for multiple and varied food genres, which are representative of both "low and high brows," to construct a 'good' personal food practice (Johnston \& Baumann, 2010: 35). These actions exemplify omnivorousness as Johnston and Baumann define it, whereby people with high levels of education and income engage with cultural tastes that sample selectively from wideranging genres to signify high status through the presentation of an 'ethical' self, rather 
than traditionally highbrow displays. My data indicates that, when applied to the construction of personal food practices, this type of 'omnivorousness' is functional in a number of important ways.

First, cultural omnivorousness permits a degree of flexibility that is critical for successfully navigating the risks and confronting the challenges of our food system. Pointing to the reflexive nature of their research process, interviewees insisted that they integrated a variety of complex and simple information to create a practice that worked for them. Thus, Ella was adamant in saying: “Again, it's not a religion, it's just a dietary choice." She gave herself permission to "bend the rules" of a prescribed raw vegan diet when it made sense for her to do so socially, or when another value (like organic) was being fulfilled. Similarly, Robert noted:

A lot of people, they find a ... 'way,' and yes, I'll put air quotes around 'way', and it becomes almost like a religion to them. Raw food, as I like to call it living, active food, is very much that. A lot of people get on that diet and become religious about it. They, they go on these strange little Jihads across Internet forums. You know, saying how pure they are, to living food, and how other people aren't as pure to living food as they are.

Criticizing this approach, he advocated an omnivorous outlook that ensures an openness to new and perhaps even more ethical ways of eating. Amelia also supplemented the knowledge she gained from reading The Paleolithic Diet with research about the chemistry of gluten-free baking to construct a personal food practice that balances good health and pleasure:

Because I believe in that science [The Paleolithic Diet], I eat less grains than I did before, even when I was gluten-free. But I refuse to cut them out completely, because what's the point in living if you can't have cake now and then 
(laughing)!? Um, um, so I make those choices. So I believe in the science, but I make modifications to suit my happiness in every day life.

An omnivorous approach to food knowledge also allows participants to be the ultimate authority on their own food practices. Interviewees consistently asserted that rather than accept the recommendations of an expert in a top-down transmission of knowledge, they 'tested' various bodies of knowledge, in isolation and in combination, to decide if that knowledge was legitimate. The information they integrated into their personal food practices was accepted (and often recommended) based on their personal version of proof, especially, feelings of physical health and wellbeing. For example, since making the shift towards a fresh food ethic, Jon reported: "I've noticed a huge increase in energy levels, less mood swings, and uh, just feel healthier overall." Katherine added: "When you make those tweaks and those adjustments, then you feel so much better. You're clear headed, you're focused, you're energetic." Similarly, Helen declared, 'I'm a person who is rarely sick. Like, just about never, and I work in a school...And I have a theory that the reason I don't get sick, is because the diet I have is very healthy." In other words, participants drew upon an "interpretive repertoire articulating the integrity of their own bodies rather than drawing upon medical authority" as a strategy (Lupton \& Chapman, 1995: 490).

Participants cited a number of examples to illustrate the 'tried and true' benefits of their 'fresh food ethic;' one participant claimed to have cured chronic stomach aches by cutting out packaged foods, another relieved recurring asthma attacks by replacing chemical-laden candy with homemade, naturally sweetened baking, and another credited her switch to organic milk with renewing her reproductive function. Participants 
integrated the core values of the 'fresh food ethic' into their personal food practices because they experienced the benefits of these values first hand and found these practices to support their perception of self as healthy eaters who maintained ethical food values.

\section{It's All Fresh}

The individuals I interviewed engaged with wide-ranging bodies of food knowledge to educate themselves on the risks of our food system and construct a highly personalized food practice that accommodated their individual interests, political leanings, nutritional goals, culinary preferences, dietary intolerances, ethical positions, and on and on. While each of my participants described a markedly unique personal food practice, I found a common thematic thread that links these individual narratives within a single overarching concept. The bodies of food knowledge cited by participants, ranging from pop-culture books by reality TV celebrities to ancient texts on holistic healing to political documentary films, disseminate clear messaging about the overarching value of 'fresh' food. Adopting an omnivorous approach to food knowledge, the individuals in my study engaged with these multiple perspectives under the broad philosophical umbrella of the 'fresh food ethic' to negotiate multiple values, address challenges, and navigate risks.

Given this finding, I argue that 'fresh' serves as an immensely valuable mediator between the multiple, interacting, and oftentimes competing interests of a personal food ethic. Freidberg (2009) argues, "of all the qualities we seek in food, freshness best satisfies all [our] modern appetites" (3). In a food system that offers innumerable options, fresh serves as a catch-all term that emphasizes the 'goodness' of the food, while allowing less 
desirable, even risky food characteristics to be left unspoken and unacknowledged. Fresh foods are pure and natural, they are healthy, they connect us to others, they are safe, they are ethical, they taste better. As long as food is fresh, that is 'enough.'

In the next chapter, I explore how fresh is mobilized for these interviewees within the context of 'dining out.' I argue that restaurants are immensely rich sites of sociological study because they constitute a closed social context wherein food values are manifest in 'lived experience.' Specifically, I illustrate how the core values of the 'fresh food ethic' are constructed, enabled, and enacted within The Green Door Restaurant. I argue that notions of freshness are employed within this space to construct an eating experience that is particularly palatable to the urban consumers I interviewed for this study. 


\section{CHAPTER 5}

\section{ANALYSIS II: Constructing Taste in a 'Fresh' Space}

In the previous chapter, I identified five core values that were cited as important tenets of my participants' personal approaches to food: local, seasonal, non-contaminated, nonpackaged, and human-handled. I illustrated how these values, each of which serves as a response to the increasing industrialization of our contemporary food system, were perceived by my research participants to ensure or enhance the 'freshness' of food. Defining 'fresh' food as that which is 'as close to its natural state as possible,' I argue that these values can also be understood as component parts of a broader narrative of 'freshness,' which operates as a catch-all qualifier of 'good' food within the urban context. I propose that because individuals cannot comply with all of their values in every food choice, and because failing to do so means that the individual has not safeguarded him/herself against the myriad risks of industrialized food production, individuals rely on an overarching commitment to 'freshness' to frame their food choices as 'good enough.' I argue that although individuals draw on widely varying bodies of knowledge to construct highly personalized food practices, all of my research participants' personal food practices can be understood within the broad frame of a 'fresh food ethic,' which provides both the cheerful positivity and the necessary ambiguity to quell fears and promote pleasure in their 'good enough' food choices.

This thesis is not only concerned with investigating how individuals identify and conceptualize their personal food values, but also with how they engage these values in 
action as food consumers. Individuals may very well describe personal food ethics that reflect the values and concerns of 'good' food, but translating these values into action is a complex, dialogical process between the individual and the social world (Feilzer, 2010). I have been specifically concerned with understanding how these values are enacted in the lived experience of dining out. Belasco notes that what we think about food (and how we engage with it) "may have little to do with the actual material properties of the food itself" (2002:13). Rather, it is the social conditions surrounding food production and consumption that shape 'good' taste. The data in my study support this claim.

One aim of my research is to investigate the 'social conditions' of food practices at The Green Door Restaurant. In this chapter, I propose that the core values of the 'fresh food ethic' are enacted in the social space provided by this restaurant in such a way as to appeal to consumers' aesthetic and ethical tastes. I see that, like the individuals I spoke with, The Green Door does not adhere to all of the multiple, sometimes contradictory values that have emerged within our contemporary food discourse. Instead, it relies on a broader commitment to 'freshness' that encompasses these values and implies a general, and appealing, allegiance to 'good' food practices. I begin the chapter by outlining the distinct and varied ways The Green Door Restaurant engages with notions of fresh to attract consumers. I then propose that the way 'fresh' functions in this space can be accurately conceptualized within an existing framework of the sociology of 'taste' (Johnston \& Baumann, 2010). Specifically, I demonstrate that 'fresh' functions within competing ideologies of democracy and distinction; it invokes egalitarian ideals of access and inclusiveness while ultimately serving as a marker of 'good taste.' To illustrate this 
analysis of 'fresh,' I draw on my interviewees' narratives about food choice in every day life, and specifically when eating at The Green Door. I show that these narratives present 'fresh' food practices as accessible and inclusive, while also indicating that such practices are exclusive, require extensive food knowledge, and meet requisite standards of cleanliness and comfort. I show that The Green Door exemplifies these dynamics for its patrons.

I then point to a dynamic that is not acknowledged in Johnston and Bauman's work on culture and taste. Whereas Johnston and Baumann argue that environmental and class issues are trivialized in contemporary food discourse, I demonstrate that individuals think critically about their food choices and recognize that their ability to act on 'fresh' values reflects a position of privilege. My data indicates that individuals do not blindly buy into market promises of status and distinction. Instead, they acknowledge the fundamentally unethical nature of the industrialized food system and engage with 'fresh' values in genuine efforts to make responsible, 'ethical' choices. This added complexity is significant because it results in a critical breakdown in the presentation of self for the urban consumer, and also ensures that the systemic problems of our industrialized food system will not be addressed. I argue that as long as individuals are pre-occupied with perpetual, and inevitably futile, consumer attempts to construct an 'ethical' self in their food choice, they cannot be engaged meaningfully in collective action. I propose that it is necessary to re-think this individualist consumer model if we hope to create a food system that is 'fresh.' 


\section{The Green Door Restaurant: A ‘Fresh’ Space}

The Green Door is perceived, and indeed explicitly described, as a 'fresh' space by those who dine there. Citing all the values outlined in the previous chapter as reasons why they go to The Green Door, interviewees consistently return to the word 'fresh' to describe the overall appeal of this restaurant for them. Many interviewees spoke explicitly about the freshness of food, and those that did not, emphasized the importance of all of the component categories of the fresh food ethic in their discussion. When asked how they would describe The Green Door, Jon said, "they are organic, they try to be as local as possible.... they have very clear indicators of what is in each of their dishes. And it is very, very fresh." Katy added, "I know, like, one thing is that some of the main dishes change according to the season. Which is something that I like as well." Interviewees also observed that The Green Door experience is peppered with activities that demonstratively showcase the local, seasonal freshness of the food. Ella noted:

One thing I really enjoyed is I often see a lot of their stuff - when the St. Paul's or the Mainstream Market was going on across the street, you'd see a lot of people just kind of, going from the restaurant there, getting stuff, and coming back, in big enough amounts that I was assuming it was for the restaurant. And, and you know, you're kind of thinking, well, 'oh, that's where it comes from' and ...And it really makes that real for you. Where your food comes from in terms of who made it, but also where it came from, like, directly.

Katy noted that the food at The Green Door is not only served with attention to the timing of the seasons, but also with optimal timing in food preparation and presentation. Katy commented that food at The Green Door is served at an optimal state of readiness, not sitting around or needing to be reheated because of poorly calculated timing: 
Like, it seems like it's fresh. And I just find that's the thing with the other places that I've been to. It seems like the food is reheated a lot. Yeah, it seems fresh. It is fresh I would say.

Jon echoed this sentiment:

And things like the broccoli are almost, like, too fresh, 'cause often you're like, waiting for them to bring out more broccoli. But that's attractive! Like, how often is food cooked, like, the minute that you're there, right? ... The avocado salad is a good example. They, the avocado salad is there, and it's a hot item, it goes fast, and when it's gone, it's gone. And so you know they're not making a ton of it, just to know that it's there all the time. I think that's a good thing! That's OK, if it's not there because it's run out and they have a commitment to freshness so they haven't made more to have it sitting around.

These participants considered the food to be particularly fresh at The Green Door because it is all consumed in its optimal state, never transitioning to a state that is past its prime. Participants were impressed that the restaurant ensures the freshness of the food for each customer by "replacing platters all the time," and they appreciated that "they're not making so much of it" that some of it goes bad:

Like it's fresh. That means it's fresh, right? And sometimes they'll say, oh no, there's no more tonight. And that's better than having, just, leftover food.

Ann sees that The Green Door offers food that is presented in an optimal state and she made a direct link between this presentation and the non-contaminated 'naturalness' of the food:

The vegetables at the Green Door are usually more al dente than I would make myself but obviously it's probably better for you because you haven't boiled out all of the nutrients. The food just to me tastes better, because it's ... all natural stuff added to it. Like they don't use artificial preservatives ... Like it's all, sort of, made fresh and so I find that tastes a lot better. 
Chloe also spoke to the fact that the staff at The Green Door make their dishes from natural, non-packaged ingredients, saying that a major draw for her is that "it's all...from scratch."

During my observation sessions, I also found kitchen staff engaged in many food practices that have been largely eliminated through the industrialization of our food system. Making dressings and sauces from scratch, preserving produce in the fall for use in the winter months, and soaking organic dried beans and lentils each day are a few of the ways staff engage in the 'human-handling' of the food to enhance its freshness (Guide to the Green Door, 2010). Describing a menu comprised of local, seasonal and organic options, made from whole, non-packaged ingredients, prepared by a "dedicated staff" and served in an optimal state, interviewees felt that The Green Door offered 'fresh' food in all the various senses of the word. Jon noted:

I know that anything I have there, whether it's from the end of the salad bar that's bread, or the desserts there... I know whatever I choose, it fits into my categories of what's good for me. It's good for me 'cause you can turn your mind off, in a sense, and not, like, when you go to other restaurants, 'is there cheese in this?' What kind of stock d you use? Dah, dah, dah...' You have this mental freedom to really enjoy everything, because you know that it's made in a way that you approve of.

As illustrated in the previous chapter, navigating through a contemporary urban food system to make 'good' choices constitutes a significant challenge for consumers. The Green Door not only addresses these concerns by providing a spread of ubiquitously 'fresh' food, but also in how it structures consumer transactions.

One of the most notable features of The Green Door is the weight-based system of food selection and payment. Research participants shared the view that the cafeteria style 
system of food selection is a valued attribute of The Green Door because it eases their worries about navigating food values by allowing them to make detailed, individualized choices about the food they eat. For one participant, this selection process meant she could choose the brownie that had, through human handling rather than mechanized processing, ended up with the most icing; for another participant it meant she could choose the piece of broccoli that was the most brightly coloured green. In other words, these research participants appreciated that they could choose food that was 'fresh' thereby engaging their own decision-making process of food selection and reaffirming their perception of self as healthy, ethical eaters.

Perhaps the most broadly shared sentiment held by my interviewees was that direct access to the source of Green Door food (i.e. the trays filled with abundant amounts of each dish) allowed patrons to enjoy small portions of many different foods. For example, Ella, who generally adheres to a raw vegan diet, liked the fact that she can take a taste of a cooked yam salad or broccoli-tofu stirfry without committing herself to a full cooked meal. Knowing that other values (e.g. local, organic, non-packaged, and human handled) were being satisfied, she felt that she could indulge in small portions of foods that had been processed beyond what she would otherwise view as acceptable. In a similar fashion, Jon noted, "I think one of the best things about the pay-by-weight system is that you can try just a little bit of everything. So many different tastes. Satisfying."

All of my interviewees spoke about the variety of foods offered at The Green Door and cited this variation as one of the restaurant's most important draws. Given the 
data analysis provided in the previous chapter, however, I suggest that the variety that Jon values so highly is not only variety in tastes, but also a variety in food values. I argue that the 'fresh food ethic' is attractive to consumers not only because it allows them to attend to multiple food values, but also because it allows them the flexibility of choice needed to enjoy the benefits of multiple values to varying degrees. Thus, I suggest that patrons of The Green Door are able to enact and satisfy multiple values with a single plate and, perhaps most importantly, feel that they can trust that any food choice they make will, in some sense, be 'fresh.' Jon speaks to this freedom in the following excerpt:

It's my favourite restaurant by far in the world, and I like it because when I go there, I.... feel that I don't have to worry about any of that. I don't have to read labels; I don't have to ask questions. I know that they've taken the care, to make sure that all of their food is A) from you know, healthy, by my standards places. And B) prepared in a healthy way.

When I asked Jon to clarify what he meant by 'healthy' he replied, "food that you know how it's grown and what care has been given to in to the growing process or the preparation process." In this way, by articulating organic growing practices, a critical approach to processed or prepared foods, and care (as opposed to industrialized processing) as core values, Jon described the food at The Green Door with the general tenets of the 'fresh food ethic.' From his perspective, the ability to select small tastes from dozens of different dishes that all fit in some way under an umbrella of freshness is 'satisfying.'

Interviewees indicated that it is not only the food that is 'fresh' at The Green Door, but also the space itself that attracts them. Specifically, the spatial design of The Green Door provides another means through which this restaurant keeps food 'as close to 
its natural state as possible.' The open concept layout of the space, with only a counter dividing the front-of-house space from the kitchen, allows patrons direct visual access to the source of their food. As patrons serve themselves along the buffet, they can look in on food preparation practices and watch the kitchen staff "kneading bread, creating desserts, stirring soups, preparing vegetables, cooking, chopping, slicing, blanching, sprouting, blending, baking, roasting, serving, juicing, brewing, wiping, washing, dishing, scrubbing and placing food on the buffet" (Guide to the Green Door, 2010:3).

In the majority of other dining institutions, servers represent the customer's only point of contact with the "backstage" staff and the activities engaged in food preparation. In contrast, rather than employing these gatekeepers as the customer's only restaurant liaison, The Green Door grants patrons relatively direct access to many conventional staff spaces and activities. In addition to serving their own food, customers bring their dirty dishes to a dish drop off area, where they engage with a space that would conventionally be off-limits to restaurant guests. Significantly, by deconstructing this front-stage/backstage divide, The Green Door engages its patrons directly in the 'human-handling' of their food. For example, Robert conceptualized this practice of bringing back his dirty dishes as a completion of the natural cycle of food production: from growing, to harvesting, to preparing, to decomposing. From his perspective, engaging in this final phase of decomposition was viewed as an acknowledgement of the fact that a sustainable food system must be cyclical, rather than linear. I would add, however, that this contact with the backstage activities of The Green Door Restaurant also serves as a measure of 
transparency, reassuring consumers that dishes are made from whole ingredients, fresh to order and that food recipes are produced by people, rather than through mechanized processes of mass production. In other words, food is 'fresh' and, therefore, ethical to eat.

The Green Door also engages with the various meanings of 'fresh' in its decor. Tina describes The Green Door as a bright, vibrant space that reflects the vibrant freshness of the food:

And that, the atmosphere, right? Like, I think it's very ... it's very clean, it's very bright, right? Everything is freshly painted, it's renovated, it has artwork on the walls. It's very colorful.

The artwork, a rotating showcase of local artists, situates the food within the context of the larger community. Like the food, this decor is local, handcrafted, and 'ripe,' brimming with colour and energy; it is not imported or mass-produced. Two community boards at the back of the restaurant, religiously visited by most of the individuals I spoke with, also contribute to the freshness of the space. These boards display large, professionally produced posters from the City of Ottawa and various businesses in the Ottawa area as well as other more informal notices from members of the community that advertise things like garage sales, potluck dinners, missing animals, or personal services. This influx of information brings the activities of the outside community into The Green Door space and situates the patrons' individual food consumption practices within a wider 'human' context of others perspectives. This is important because it grounds food consumption at The Green Door in notions of communal action; it suggests that creating a socially responsible, ecologically sustainable food consumption is a collective interest, 
rather than an individual endeavor, and that the broader goals of eating 'good' food are for a greater social good, as well as for individual health and wellbeing.

Participants also noted that food consumption at The Green Door is enlivened by the restaurant's creation of a more communal dining environment. The physical construction of the dining space is such that patrons are positioned in close proximity to one another. Tables are arranged densely, and benches, which line the outer wall, are often shared by the patrons at a neighbouring table. A number of interviewees reported that spontaneous interaction with other patrons increased the value of the Green Door experience. For example, when I asked Robert about his interactions with other patrons, he said:

Really, [the topic of conversation] could be anything! Uh, it could be the conversation they were having, something they're wearing...Because other people will initiate the conversation as much as I will initiate conversation.... it can be about the book I'm reading. Really anything. And it's that openness, that ability just to talk to the person beside you, that I find very rare.

Robert believed these openings to connect with people during food consumption mirrored an openness around food practices, more generally, and extended the 'human touch' (a priority in food production) into the final stages of food consumption. Although all interviewees did not report this type of exploratory interaction between patrons in different parties, the reality of the physical space at The Green Door is such that patrons are almost inevitably implicated in one another's experiences in some way. Patrons can hear one another's conversations and can easily observe others' behavior within the welllit, open concept space. They may also interact with one another in basic capacities 
unlikely to be seen in many other restaurants with serving staff, such as asking another patron to pass the salt, or to shift their belongings so they can sit down at the other end of the same table. Importantly, my interviewees considered this physical arrangement to be an asset. As one interviewee noted, "I like the kind of, communal environment. I like the fact that it's very ... There's sort of a community there.... or culture ... people tend to be friendly. And it's lively." From their perspective, the lively communal atmosphere of The Green Door enhances their eating experience, with noise, colour, and community highlighting the raw energy of the food. In contrast to the highly individualized, anonymous consumption that takes place at many conventional food retail spaces, this spatial arrangement contributed to their sense of the food as 'fresh,' and therefore, more pleasant to eat.

The Green Door's physical space is also viewed as enhancing food consumption because it integrates other environmental elements as part of the eating experience. For example, the entire street-side wall of the restaurant is lined by huge windows. These windows allow the larger context of Main St. to 'enter' the restaurant, including the activities of passersby and the natural elements of the day. This intimate interaction with the external physical environment reinforces the 'human' element of the fresh food ethic, which insists that food be situated in social, rather than industrial, practices and processes and attunes one's food consumption to the natural processes of shifting seasons. Additionally, staff create a seasonal environment within the restaurant's physical space. Thus, for example, in the spring, dining tables are decorated with fresh, seasonal flowers. Potted plants, including a Bonsai tree, and jars of rocks and seeds are also incorporated 
into the decor thereby grounding the activities at The Green Door in natural elements. As such, Green Door patrons encounter a physical context that reinforces their sense of 'fresh' year round.

My participants indicated that the value of The Green Door Restaurant is that it offers a 'fresh' dining experience. While this need may seem relatively straightforward (customers value a, b, c, d, e, so restaurants sell an experience that includes all these values), I argue that engaging with notions of 'freshness' to construct a commercially viable dining experience reflects a complex and significant trend in contemporary urban food consumption. Specifically, my analysis builds on Johnston and Baumann's (2010) analysis of shifting food values in gourmet food discourse. These scholars argue that new markers of high-status food have emerged within contemporary food discourse. They illustrate how notions of authenticity and exoticism have become prevalent in gourmet food discourse in "a general trend away from snobbish exclusion towards cultural eclecticism by high-status cultural groups" (35). This trend is significant because authentic and exotic foods seem to stand for food practices that are accessible and inclusive, while actually representing an approach to food consumption, which valorizes foods that are inherently difficult to come by, expensive, and exclusive. Based on my research findings, I argue that 'freshness' functions in much the same way. Freshness occupies a role as mediator and consolidator of local, seasonal, contaminant-free, nonpackaged, human-handled values serving as both a democratizing force and an indicator of distinction. In the following sections of this chapter, I will demonstrate that freshness 
invokes democratic ideals of accessibility and inclusiveness, while simultaneously attracting consumers with promises of quality, cleanliness, indulgence, and exclusivity. I argue that it is immensely useful to conceptualize freshness within this framework of analysis because the concept of fresh clarifies and highlights the tensions that now characterize particular individuals' attempts to choose 'good' food.

\section{Fresh: Democratizing}

Many food scholars have explored the notion of 'democratized' food consumption (Johnston \& Baumann, 2010; Lang, 1998). In the broadest sense, a democratic ideology supports the consumer's capacity to actively participate in shaping processes of food production and consumption. Framing food as commodity, this ideology holds that shopping for food exists within "a realm of freedom from work and politics - a form of democracy open to all" (Zukin, 2004: 43). Johnston and Baumann (2010) specify that democratic ideology is "organized around normative liberal principles of human equality and meritocracy" (41); it implies that individuals make choices based on their personal values with access to options, equal opportunity, and freedom to manifest choice into action. These scholars also note that a democratic ideology is connected to populist ideals that frame the United States (and Canada) as a classless and multi-cultural society, where "immigrants of multiple races and ethnicities have equal opportunities for socioeconomic and cultural advancement" (41). Many food scholars point to shifts in food production that seem to facilitate this type of democratization (Roseberry, 1996).

Globalization of trade and mass-production of food goods through processes of industrial 
mechanization have resulted in a contemporary food system that does indeed offer unprecedented access to a seemingly limitless supply of food for a majority of Canadians.

Within this context of greater availability and access, the ways we talk about food, and the types of food we value as 'good,' have shifted. Johnston and Baumann illustrate some of the most significant shifts, charting how the valorization of French haute cuisine has been replaced by the valorization of adventurous pursuits of the exotic and earnest quests for the authentic. They argue that the overt display of these values in gourmet food writing reflects the declining acceptance of old-fashioned snobbery and a growing disapproval of celebrating of high status based on wealth, social position, or ethnic/racial superiority. In this section, I argue that, like authenticity and exoticism, freshness invokes democratic ideals of accessibility and inclusiveness. Drawing on my interviewees' accounts of their personal food practices, as well as their specific comments about The Green Door, I illustrate how conceptualizations of 'fresh' are employed as a marker of 'democratic' dining.

\section{Fresh as Accessible}

One of the most prominent sentiments that emerged from my data was that 'good' fresh food is accessible. Specifically, 'freshness' was viewed as available, casual, convenient, and affordable. Ann articulated the shared view that 'fresh' options have become increasingly available since she began being food conscious:

No matter what end of town you're in, there's likely going to be a health food store, or a section in the grocery store that's organic, or a restaurant that's totally organic, or at least mindful that people have food allergies and sensitivities. 
Within this context of abundant availability, fresh is viewed not only as a choice, but an easy choice. For example, Katherine was adamant that people can make 'fresh' choices, despite popular perceptions that this is not the case. Offering the story of a woman at her gym who said that she cheated on her diet because she was busy shopping all day, Ann exclaimed: "She's in Costco and she thinks all she can have to eat is a slice of pizza. But: You're in Costco! Go get a salad. They're right there!" Katy clarified that although the salad would not likely be organic, it was a more 'fresh' option because it offered ripe ingredients that had not been processed. Helen also viewed 'fresh' in terms of simple, common sense choices:

I'm an apple a day person. We always have fresh fruit at home. And fresh vegetables. Like, we will literally munch on radishes or carrots sticks or apples. For Helen, eating 'fresh' is about following the simple, age-old formula of 'an apple a day.' Ripe, non-processed, non-packaged, and in this case organic, snacks are presented as quick, easy, nutritious solutions that are accessible to anyone.

The accessibility of 'fresh' was also articulated in terms of casual (as opposed to formal) dining. Participants expressed a shared belief that they did not need to go to a 5 star candle-lit restaurant to eat 'good' fresh food. Rather, fresh could be found at any family-style Tuesday night dinner or even, as Katherine indicated above, in Costco. This perception of casualness reflects an overall approach to eating that values joyful excitement for food over poised critiques. Johnston and Baumann (2010) point to this trend in their work and highlight unbridled passion, sensualism, and enthusiasm as core tenets of the 'foodie' identity and contemporary gourmet discourse (60). Similar to 
Johnson and Bauman's study participants, my research participants were adamant that their interest in food was about enjoying the simple pleasures of eating. Yes, they liked to try new things, and of course, taste was important, but there was nothing 'fussy' or pretentious about their tastes. Thus, Erin admitted that she is known among friends and family for her eager, non-discerning appetite, and said, "Like, I've heard, like people say, like if a steak is cooked more than medium-rare, it's ruined, forget about it. You know? And I'm like, no, it's still steak. It's good. You know? Don't waste it." Here, Erin argues that she associates good 'fresh' food with gobbling up every last bite of steak at a backyard barbeque with friends. She then contrasts this memory with the story of her attempt to indulge in the finer pleasures of French cuisine:

We went to a very fancy restaurant in Paris. We thought, this is great. If we're ever going to try an oyster, this is the place. I swear, we were sitting, we were dressed up, we were sitting next to, like this woman who looked like absolute, like ... Grand Dames, you know what I mean, with the fur around (gesturing around her neck), their air, very dignified, speaking very French. And it wasn't a family restaurant. This was like, I think it might have even had a Michelin Star. I'm not sure. Anyway, so we went in, and I said, OK, we'll get the Table D'hôte, and I'm going to try the oysters. I'm kind of gagging right now, thinking about it. I tried it. And I almost threw up. It was so gross.

Making a sickened face and bringing a napkin to her face, Erin reported she had to chug back her glass of wine to get the taste out of her mouth and laughed happily about her inelegant introduction to this quintessentially 'gourmet' food. While Erin believed the food at this restaurant was 'fresh' in the sense that it was prepared thoughtfully by staff and was served in an 'optimal state,' she did not perceived it to be any better than the fresh food prepared at the barbeque. In fact, Erin valued the more casual meal because it 
was imbedded in the 'human' context of her friends and family and she could indulge fully in the pleasures of the food. In fact, Erin noted her preference for simple, casual food at numerous points in the interview, describing, in an increasingly lilting dialect, the good, hearty fare of her Maritimes hometown. For example, when I asked her about her favourite foods, Erin said:

Like, boiled dinner. Not exactly haute-cuisine, but it's still so good. ... You take salt pork. Salt beef, more salt beef normally. And then you boil it all day, but you change the water a few times, so that they take the salt out of it. And then it's nice and tender. And then about an hour before you're ready to eat - or an hour and a bit - you add um turnip, potatoes, carrots, and then a little bit - just before the end - you add a, put a half cabbage on the top so it steams.

This simple one-pot recipe, a day-long process carried out by her grandmother, represented the 'goodness' of traditional homemade food for Erin. The dish was framed as fresh because it was made from local ingredients and hand-made by a thoughtful and loving cook. However, Erin also conceptualized 'goodness' in a personal way that drew upon positive memories of family and her past eating experiences. Thus, Erin indicated that she took great pleasure in eating this 'everyday' food, adopting a devilish grin and adding: "MMM! Then you take it out and drench it in vinegar!" In a similar fashion, Erin noted that most of the foods she ate as a child were fresh because they were local:

So really down there everything is pretty much local any way. It's just not an effort to make it local. It just is... Like the wharf is literally just down the road. So we would go down and get fish in the morning and like, eat it.

Reminiscing about how her grandmother would cook a rich seafood chowder with the freshly caught fish from the wharf as a weekday meal, Erin illustrates the strong belief 
reported by my other interviewees, that as long as it is 'fresh,' food does not need to be 'fancy' to be good.

Helen provided a comparative example to Erin's, using her husband's daily dinner offerings as evidence that fresh hand-made, seasonal meals were delicious, but not "presumptuous:"

We eat a lot of pasta, lots of sauces, vegetable sauces, um, lots of legumes, lentils, various beans. Uh, lots of cooked greens, potatoes, rice, uhm, we call it, we baptized his style 'wholesome hearty'. Wholesome hearty. There's nothing, nothing presumptuous about it. It's comfort food.

In this way, my interviewees agreed that fresh food can be casual, rather than formal or pretentious. However, in taking this view, they also engage with the democratic ideology that everyone has access to the types of food that they value.

My research participants agreed that one of the biggest draws of The Green Door was that it is casual. Rather than trying to fit in with the 'grand dames' of France, Erin, and other participants, said they just wanted fresh food in a casual restaurant:

It's not ... it's very functional. It's functional. Nothing pretty about it. Nothing that's like, let's dress up and go to the Green Door. No! It's like, let's go there in my sweaty yoga clothes to get take out.

Ann also commented on the casual dress of the "granola eater, tree hugger" patrons:

Just seeing women go in there that have not even done their hair. And it's disheveled, and they have, like, home-made knit socks on and they're wearing their Birkenstocks.... and maybe, like clothing that's from a second hand store.

Chloe remarked, "It's not, you know, it's not a nice meal out where you're going to a sit down restaurant, but at the same time, you know, it's tasty, it's fun, it's convenient." Additionally, many research participants noted that a major attraction of The Green Door 
is that you can get in and out as quickly as you need to; they could come in for a quick 15 minute meal between meetings, or run in for 30 seconds to fill a take-away container after an evening yoga class. Thus, Tina said:

You know, so it can be somewhere that you're just going for a really quick meal and you just need to, you know, in and out, 'cause you serve yourself, and you know, like I've gone to eat at The Green Door in like 20 minutes, and you're gone.

The lively atmosphere at The Green Door, and the convenient cafeteria-style system of food selection and payment, reflect the 'faster-is-better' values of contemporary Western culture. In an increasingly fast-paced, overly scheduled culture, having fresh, convenient options is attractive to the individual who wants to make 'good' choices.

Chloe adds to this perception of The Green Door's appeal of being easily accessible with her suggestion that the lively atmosphere at The Green Door also reflects the fresh 'goodness' of the food offered:

[The Green Door is] easy going. It tends to be more lively. It's louder. Often there's more chit chat going on. People seem excited to be there, whether that's the food, or the atmosphere, or the people that they're with.

This casual, easygoing atmosphere is created in large part by the physicality of the Green Door space, which resembles a cafeteria-style layout. While participants seemed aware that many people might dislike this format, wanting instead to "sit at their own table, in their own space and have people bring them stuff" many cited this layout as one of the primary draws of the restaurant for them. Katherine said: "I personally like it. Um, because it's easy, it's casual." Tina made the link between the values of the fresh food ethic and a serve-yourself format. These types of comments made by my research 
participants reaffirmed my assessment that this egalitarian format of requiring all patrons to 'get in line' and offering all patrons (regardless of who they are) equal access to all the same foods challenges the inequities of conventional server-patron power dynamics.

Thus, for example, when I asked her what she likes about The Green Door, Tina replied:

It's a few different things. So obviously it's vegetarian, so that goes with my ethics in that sense. And the local, organic I like. But I also really like that it's a serve yourself thing. In terms of... limiting the hierarchical structure of restaurants...I don't need to have someone serve me... it seems very constructed and like, a facade. Right? ...I kind of like that they have, they just put the food out ...I love, love that when people come out from the kitchen and they put food out, they don't wait they're just like, 'Excuse me! I need to put the food here!' And I like that! You know, in restaurants there's often like, the customer's placed on a pedestal, whereas the staff are way lower.

Similarly, Robert noted that Green Door staff contribute to the 'freshness' of the Green Door experience in that they are "very friendly, very open, very genuine people." While others reported only a minimum of civility from Green Door staff, all agreed that "there's very little pretense" in their interactions with patrons.

Goffman (1959) explores such pretense in his book, The Presentation of Self, when he employs the example of the Shetland Hotel to analyze the front-stage/backstage divide present in most social institutions. Specifically, he describes a conventional restaurant set up, where the owner insists on keeping the kitchen door closed in order to conceal the disorder of cooking preparations from upscale clientele. By removing this boundary, The Green Door not only provides consumers access to the inner-workings of the restaurant, but it challenges this power dynamic of maintaining control of the food process involved in a consumer's eating experience. In contrast, rather than hiding away 
the messy preparation, The Green Door showcases the human labour and creative energy that goes into preparing fresh food and enables its patrons to support, critique or question that preparation. In consequence, Helen sees that Green Door staff - equal to, rather than in service of, Green Door patrons - are valued with fair wages and job satisfaction. This perception complements her sense of self as an ethical food consumer. To quote Helen:

There are many who have been there for a long, long time. Like the people - I'm thinking of one person in particular that I've seen in the kitchen over a number of years - he's been there, as long as I can remember. So, this tells me, there's job satisfaction, they probably have a good, a fair wage. You know, because otherwise you wouldn't be able to stay there that long. So this, this all speaks, speaks to the kind of values that are important to me. You know, treating employees with respect.

Jennifer also appreciates this alternative approach and is willing to pay extra for it:

I know what goes in to making the food. And so I see it as very good value. I've also worked in restaurants and I know that this kind of food that they're making is extremely labour intensive. And so I understand the connection between wages and the price I pay for food as well. Not just the quality of ingredients. So the price doesn't bother me that much.

Although interviewees acknowledged that eating at The Green Door may be perceived as expensive, all indicated that the fresh food they received was well worth the price. A number of them argued that they were paying the 'real' cost of eating good food. When I asked Helen why she goes to The Green Door, she immediately replied, "Hmm, because it's the best restaurant in town! It's got the best bang for your buck."

These types of attitudes concerning the cost of food provided at The Green Door were reflected in the interviewees' overall perception of the steps they were willing to take in maintaining their sense of self as ethical eaters. Many of the individuals in my study noted the financial challenges of eating 'good' food, but all concluded that the 
expense of fresh food was better value than cheaper alternatives. For example, Helen insisted throughout the interview that fresh food is affordable: "We eat on a shoestring, but that's because of the genius of my husband. He really knows how to stretch a dollar." When I challenged her on how much she had to compromise on freshness to stay within his budget, asking for example, if she bought many canned foods, she replied passionately, "No! No, not at all! ... No! No, no, no! Everything's raw. Everything's real."

All but one of my research participants commented on the limitations of their financial resources, but all described consistent participation in consumption practices that reflect the values of the 'fresh food ethic.' As such, my data demonstrates a consensus that good 'fresh' food accessible; it a casual, everyday option that is convenient, affordable, and, moreover, reflects non-hierarchical power structures that promote equal access to 'good' food.

\section{Fresh as Inclusive}

The interviewees' desire to participate in consumption practices that rely on less hierarchical power structures relates to another important sentiment of the fresh food ethic. Musing on the value of The Green Door Restaurant, Chloe offered:

It's about...the inclusiveness of it too. Like there's a lot of different types of people that go there as well. So it's a very inclusive cultural environment. You can see young families with kids, you can see older people. You can see people who... um ...who have the outward appearance anyways of kinds of beliefs. So you see people who look like they used to be hippies, or still are. One of the kids that works there sort of looks like an Emo kid. And you know like, it's just sort 
of very.... fairly diverse. But you also see, sort of, straitlaced people or students eating there too. Sitting there, eating, too. So it's kind of a big cross section.

Many interviewees noted that the Green Door seemed to be a place where all types could gather. When I asked “Who goes to The Green Door?" interviewees listed high school students, staff from St. Paul's university, seniors, business people in three piece suits, young couples with babies, yoga instructors, and on, and on. Katherine commented, "It's one of those places that kind of runs the gamut." This variety in The Green Door clientele was significant to the research participants because it suggests a democratic ideal of equal access to 'good' food options. People of widely varying ages, ethnic backgrounds, professions, and lifestyle choices are able to make 'good' food choices within this space.

Interviewees noted specifically that the variety of cultural influences in the buffet contributed to the inclusiveness of the space. From their perspective, The Green Door's offering of a spread of food dishes that includes Indian curry, lasagna, quiche, kimchi, sushi, New York-style (tofu) cheesecake and Greek salad (to name only a few) creates a space that invites people from varying cultural backgrounds to come and share in a communal consumption experience. As such, it supports the democratic ideology that "fuels the omnivorous notion that arbitrary standards of distinction based on a single, elite French notion of cuisine are unacceptable, and that multiple immigrant ethnicities and working-class cuisines possess their own intrinsic value" (Johnston \& Bauman, 2010: 41). While this view does not acknowledge the notable absence of clientele of low socioeconomic status (which will be addressed in a later section), it does reflect the 
interviewees' consistently shared, perception that 'good' fresh food is a unifying and attainable value across varying life stages and life styles. As Chloe observed, "It doesn't set up barriers for people.... All kinds of people." Green Door patrons come from varying 'walks of life.' They represent food consumers who would likely not congregate regularly in other food consumption spaces, but who can come together in happy coexistence within the context of 'fresh' provided by this restaurant.

Interviewees indicated that an important aspect of the inclusiveness of The Green Door relates to its ability to minimize differences in ideology around food consumption. They observed that, although The Green Door is a vegetarian restaurant, it is patronized by eaters (including many of those I spoke with) who self-identify as everything from omnivores to pescitarians to raw vegans. A number of my interviewees viewed this minimizing of differences as significant, reporting a tendency for vegetarians (and other restrictive eaters) to express a righteous snobbery about their food choices. Renee described this sense of snobbery in her interview when she said,

I find in this community of non-animal product eaters, there's a little bit of oneupmanship sometimes. Like, you find that 'oh, well you eat yogurt, well then you're not a real vegetarian.' I find that sometimes if you get in to a conversation with people who are not animal product eaters, you can sometimes feel like it's a competition about who eats the best.

While Renee reported consistently experiencing this type of judgment in other circles, she noted that such snobbery appeared to be absent among eaters at The Green Door; instead, all types of eaters are welcome to share in 'fresh' food in this space. In fact, Robert expressed gratitude for those who eat according to this less regimented, more 'loose' food philosophy. He credits flexitarians with enabling him to eat 'good' food and 
welcomes them into the space in which one's own sense of self as an ethical eater may be more easily accepted. To quote Robert:

One of my sister's friends described herself as a vegan who enjoys an occasional steak.... And really, god bless the flexitarians. Because vegans and vegetarians are less than $3 \%$ of the population. And all the vegan and vegetarian food in restaurants that we enjoy, would not be able to survive being supported by that small percentage. Flexitarians are about $15 \%$ of the population. So, god bless them, because if not, the rest of us couldn't be vegans or vegetarians. They actually make it possible for us. A lot of people say they're oh, they're dilettantes and they just don't have the where with all, and it's like, no, I bless them ever day because they make my life possible.

Jon offered a suggestion as to how The Green Door facilitates this sense of inclusiveness in his statement, "Because it's the pay-by-weight system, I think nonvegetarians feel safe to go in there as well." Significantly, Jon argued that having control over what is placed onto one's plate gives more people opportunity to experiment with making food choices that are all 'good' in some, if not all, senses of 'fresh.' To illustrate his point, Jon related the story of bringing his most 'meat and potato' friend to The Green Door. This person piled food up on two large plates and ended up loving the food so much he bought the cookbook. These actions suggest that regardless of personal philosophies around the consumption of animal products or vegetarian eating everyone can share and enjoy a meal at The Green Door.

Interviewees indicated that this inclusiveness is valuable because it allows all types of eaters equal access to 'good' choices. More important, individual participants can draw support at The Green Door from a range of others who share 'fresh' values. As Robert admitted:

In my experience vegetarianism and veganism tends to be that very sort of lonely thing that you do, sort of at odds to your family. And you have to explain it to 
your family, and you have to apologize to your family. And ultimately, your family, the best thing they can do is understand.

In contrast, Robert feels accepted at The Green Door, enjoying a sense of belonging with other eaters who hold similar food orientations. Interestingly, Robert noted that although he does not share a food philosophy with any family members, his sister does come with him to The Green Door. Many other interviewees also said they came to The Green Door with their families because it was an eating option that everyone could enjoy. To quote Tina:

Finding places that are mutually enjoyable for everyone involved is ... 'cause yeah, my parents and my sister are not vegan, so places like The Green Door are perfect, 'cause you know, my dad can still have lasagna, right? Like it's still familiar foods, and the ambiance is something that they're comfortable with ... and then it still provides us with the type of food that we're interested in.

Feeling supported in making 'good' food choices was important to my research participants and whether this support came from family or other Green Door patrons, participants felt as if such support always existed for them at The Green Door. In fact, some participants went as far as to say that fellow Green Door patrons feel like family:

There's this mix. It feels ... quite frankly, it feels like an extended family, that you don't get very many places. Especially like, think of the restaurants most younger people go to...the feeling you get is people are there more for themselves, for their own pleasure; well, of course we're all out for our own pleasure. But it's a different mood. It's more of a family feeling.

In this passage, Helen highlights an important tension between personal desire and social responsibility. Like many other research participants, she speaks to the necessity of making food choices that are not only good for the individual, but also for society as a whole. Importantly, the participants in my study indicated that the 'fresh food ethic' 
permits easier expression of both of these priorities. For example, having spoken to the tenets of 'fresh' throughout our interview, Robert explained that his desire to make socially responsible choices outweighs his personal tastes:

Say for instance I find a food that is particularly healthy for me.... but I find out that the supply structure is damaging...Yes, this might be something that is grown sustainably and organically and produced with love, but if it's shipped from China, I have to reconcile those food miles. And if I can't reconcile those food miles, I might have to find a new source or an alternative food.

To summarize this perspective, Robert concluded:

What comes down to for me, is the maximum amount of health, and the maximum number of systems, to the maximum number of people. And that's what's important. And that ability to adapt as you go along in order to change to change our circumstances.

Similar to the majority of my research participants, Robert wants a food system that is good for everyone; a food system that is inclusive and reflects the shifting values of the people engaged in that system. This populist sentiment aligns with a democratic ideology, honoring and incorporating the needs of everyone by choosing 'fresh.' Given the structure of the urban food system, however, compromised choice becomes a necessity. The Green Door offers its patrons more effective opportunities to assert their freedom of choice and more effective expression of their democratic ideologies.

\section{Fresh: Status \& Distinction}

Johnston and Baumann (2010) argue that the democratic ideology that characterizes contemporary food discourse operates in dialectical tension with an ideology of status and distinction. They suggest that overt ideals of liberal democracy, like accessibility and inclusiveness, reflect only a faux populism that operates on covert underpinnings of 
high-status cultural distinction (41-42). They illustrate that rather than reflecting any genuine shift towards an egalitarian food system, valuing authenticity and exoticism have become new ways for those individuals with economic and cultural capital to make cultural selections that demonstrate 'good taste.'

I argue that the 'fresh food ethic' exemplifies this ideological tension. Freidberg (2009) writes that "the qualities that make freshness seem natural have also traditionally made it a marker of wealth and social status" (7). Offering examples of Roman senators stocking artificial ponds with exotic fish, English gentry dining on fresh game from their hunting grounds, and French aristocrats adorning their tables with fresh peaches and pears from private orchards, Freidberg argues that the "conspicuous consumption of freshness" has long reflected - and perpetuated - social hierarchies (7). My research indicates that 'fresh' functions as a marker of status and distinction within The Green Door space. Specifically, I found that beneath the overt construction and widely shared perceptions of accessibility and inclusiveness expressed by my research participants, Green Door patrons engage with 'fresh' in a social eating space that must ultimately be understood as exclusive, as a means of demonstrating discriminating tastes.

\section{Fresh as Exclusive}

The most glaring challenge to the perception that The Green Door works within a democratic ideology of accessibility and inclusiveness is the fact that dining in this restaurant is expensive. For example, Katy observed, "I don't really go to The Green Door. Not that often. And I can tell you why right now. Price." 
Food is priced by weight at The Green Door at a cost of $\$ 19.25$ per kilogram for hot foods and salads and $\$ 22.50$ per kilogram for desserts. Depending on the plate construction of the patron, The Green Door can be an immensely expensive dining establishment. When asked what she expects to pay for a meal at The Green Door, Katy said, "I would say $\$ 15$ to $\$ 17$ dollars ...With dessert I would say closer to $\$ 17$."

My research participants cited typical plate prices from $\$ 8$ (for light lunches) to over $\$ 30$ (for a dinner). Importantly, customers do not know the price of their meal until after they have selected it. Dining at The Green Door not only requires sufficient financial resources to pay for a $\$ 20$ lunch, but also necessitates a degree of disposable income that allows a patron to gamble on a pay-by-weight price that may be more than estimated. The story of Jon's first visit to The Green Door highlights this point:

I very clearly remember the first time going there because they said I set the record for most expensive plate. My first plate ever was 38 dollars? And my mother was horribly embarrassed, but it was a good thing she was paying. (Laughing) But I had to be more careful when I was buying. ${ }^{8}$

Tina shared similar thoughts: "So it's expensive to, to go there for sure, compared to other restaurants that would maybe serve similar things...right? Like if you went to an Indian restaurant and had, like curry, or if you went to a sushi place and had sushi." Tina stressed that the value of The Green Door is its engagement with the values of the fresh food ethic; there are various restaurants that serve similar types of dishes, but they are

\footnotetext{
${ }^{8}$ I observed many incidents similar to that which Jon describes during my field excursions. While it may be that this system produces shocking results for patrons who come to the restaurant for the first time and never return, the Green Door patrons I interviewed were sufficiently committed to their food ethic and to the Green Door to choose to participate in this 'gamble' again and again.
} 
inferior because they are not as 'fresh.' Making the link between price and socioeconomic status, Erin noted:

'Cause someone who's on minimum wage, renting an apartment, and trying to go to school, probably isn't going to pay for a $\$ 15$ lunch. You know? When you can get a $\$ 5$ sub. Really, I mean, that's the economics of it. So, I think it works well in that socioeconomic group, of Old Ottawa East, because people can afford to eat there, and they want to take the time, to go out of their way, to eat better.... So the people who frequent it are most likely to be in the upper class, upper tax bracket kind of guys.

She also commented:

It's also catering to a specific ... Um, market, of people who are aware. Because wealthier people tend to be a little bit more educated and they also tend to be more, be able to afford food to take care of themselves better.

As Erin articulated, The Green Door excludes individuals of low socio-economic status on a number of levels. This outcome may be unintentional but it does reaffirm Freidberg's analysis of 'fresh,' which highlights the historic relationship between fresh food and exclusivity. Freidberg (2009) offers a number of examples to illustrate how fresh has been implicated in highbrow displays of good taste; French aristocrats ordered the construction of greenhouses and chilled storerooms so they could serve 'fresh' fruit when it was most costly and scarce, and Roman senators imported barges full of live seafood from distant waters to dine on with guests (7). My data indicate that this link between freshness and affluence persists in contemporary food practices. However, consuming 'freshness' at The Green Door may also be considered 'exclusive' in another capacity; I see that dining at this restaurant not only requires considerable financial resources, but also food knowledge to inform and display "good" taste. 


\section{Food Knowledge: Evaluating Quality}

The interviewees in my study demonstrated the idea that 'fresh' is not only an indicator of 'good' food, but also a reflection of good taste in their accounts of why they patronized The Green Door. As previously mentioned, they read labels, conducted research on food, bought special food brands and spent much time in food selection and food preparation. In this way, these research participants conceptualized how fresh functions as a marker of status and distinction for them. Specifically, they demonstrated (both explicitly and unknowingly) their social status as educated and evaluative eaters who approach food as informed critics with clear standards.

Interviewees overwhelmingly agreed that the food at The Green Door is of the highest quality. To offer one participant's 'review:' "That freshness. That commitment to quality is always there." In a similar fashion, Jon noted that The Green Door meets and exceed his standards, pointing specifically to the expert skills of staff who produce food that is in its optimal state:

We can all make a stir-fry, but [the broccoli tofu stir fry] is just done so perfectly. Like, the broccoli is so firm. And the tofu is just like, just the right consistency of chewy-ness, and the sauce is subtle, but delicious.

Helen offered her husband's hard-won loyalty to The Green Door as a testament to the superior quality of the food:

He is highly critical of restaurant food. Which is basically the reason we don't go to any other restaurants. Because it's not pleasant for me to just listen to him criticizing everything (laughing). And that never happens at The Green Door, so it's a happy experience! ... It's so incredibly rare that we go anywhere else!

Having spent years as a professional chef who cooks exclusively from "all fresh ingredients," Helen offers her husband's approval as a testament to the high quality of 
food at The Green Door. It legitimizes The Green Door as a high quality dining space for her. Crediting their use of organic, perfectly ripe, hand-made, seasonal dishes, Helen said: "Never, ever, ever has he criticized the food there." She notes that her husband's current enthusiasm for The Green Door is particularly interesting because he has not always expressed openness to the range of culinary influences present at The Green Door:

My husband has grown considerably in his appreciation of foods from elsewhere. Because he was raised in a mono-cultural situation, where everyone just ate the same. Unlike here in Canada, where, we're from all over the place.

In this way, Helen's description of her husband's shifts in food acceptability and his new attitude toward the food provided by The Green Door exemplify a larger shift towards cultural omnivorousness whereby an appreciation for multiple, varied influences, as opposed to appreciation for a single high-brow genre, has become the accepted way to achieve and signal cultural status (Johnston \& Baumann, 2010: 35).

Customers of The Green Door are required to navigate through a buffet of unique dishes that draw on ingredients and flavour combinations from a wide range of culinary traditions. My participants revealed a remarkable competency in identifying and appreciating the specific value of each dish. For example, Robert demonstrated specialized knowledge when he outlined his selection strategy:

So for instance, I will have kimchi. And I'll always eat that because of the beneficial effects on the diet and with digestion. Kimchi has very good probiotics and its spices are aiding to the digestion, so I take kimchi and I'll eat it last. Kimchi is really strong, so if you eat it first, you don't taste anything else. 
Robert is able to navigate through The Green Door buffet with ease because he knows the nutritional properties of the food and the standard protocol for consumption. Although dishes are labeled, the signage presumes a level of literacy around fresh food that reflects 'insider' knowledge of the core tenets of the 'fresh food ethic.'

These Green Door patrons not only understood the scientific, naturopathic, and social relevancies of, for example, a "gluten-free" product but had also investigated the relevance of this dietary restriction for him or herself. Thus, in my interview with Amelia, who has adopted a strict gluten-free diet, she explained the chemical function of arrowroot and xanthan gum used in gluten-free Green Door baking. Similarly, when I asked Katherine if she is familiar with any of the more exotic flavourings listed at The Green Door, she said that yes, she learned how to use Mirin, a sweet Japanese vinegar, while she was living in Japan. Similar to Katherine, for many participants, international travel has informed and shaped their culinary appreciation.

This worldly knowledge serves as a mark of distinction, allowing them to demonstrate a nuanced appreciation of the 'exotic.' This type of experience is particularly significant because, on the one hand, it reflects an egalitarian openness to the value of different cultures, while on the other hand, it often reflects gross inequalities between the affluent tourist (who demonstrates his considerable financial resources by engaging in costly travel) and the 'authentic' subject. It also reveals the economic and cultural capital available to these urban consumers in enacting their food ethic and achieving their quest for 'fresh' food. 
In addition to drawing on extensive food knowledge to engage with unique and obscure varieties of 'fresh' food, a number of interviewees indicated that they can appreciate the food at The Green Door because they have a broad palate. While the use of the term 'palate' may suggest the stuffy, out-of-date pompousness of a connoisseur, a broad palate is celebrated in an omnivorous era as traditional high/low brow divides are replaced by an openness to appreciate multiple influences and tastes. As a group, my research participants describe self falling within the second definition of broad palate. For example, Helen asserts:

You're always going to be satisfied because you choose what you want. You choose how much of what you want, and the variety is sublime, and the quality is guaranteed. You cannot be disappointed if you have a broad palate, which we do.

Similarly, when I asked Renee to describe The Green Door, she responded, "Really varied. There's stuff for every kind of palate. And it all looks really fresh." In consequence, while participants insisted that they appreciate the full scope of food flavours, they also indicated that they value a discerning palate. As such, a number noted they enjoy going to The Green Door because they can test their ability to de-construct and decipher the tastes in each dish:

We often, um, um, perceive cilantro, for instance, 'cause they use it quite a bit. Sometimes we don't recognize a spice or an herb, and we will ask, or we will guess - one of us might guess it. They sometimes use cayenne pepper, but of course they have to be careful with that, because some people are sensitive to that. Um, what is some of the other stuff they use.... Yeah, they sometimes use turmeric, or, or some of the Indian spices. Um, ok coriander, that's said. Cilantro...oooh, and dill of course. They use all the fresh herbs. And very, very well. 
These Green Door patrons also demonstrated their discerning tastes by commenting on the aesthetic appeal of the food. Research participants agreed that The Green Door's artful approach to food is evidenced not only in terms of recipe development, but also in the final presentation of dishes on the buffet. Food is heaped in bountiful display, appearing endlessly abundant through a well-kept schedule of re-filling dishes. The food creates a rainbow of colours, glistening brightly in a display of eclectic variety. A tower of meticulously rolled brown rice sushi, a mountain of bright orange yams shimmering with sesame oil and flecks of seaweed, and a fan of snappy green asparagus drizzled with olive oil and sea salt are just three examples of the dishes that make up this colourful collage. As one patron said of the avocado salad, a particularly popular dish: "I just don't know how they keep it looking so beautiful and green.... it is all so beautiful." Similarly, when I asked Renee how she would describe the food at The Green Door, she exclaimed: "[The food is] varied in terms of taste. Varied in terms of colour! Like, you look at the buffet and you're just like, wow!" Julie said that despite being an avid meat eater, she eats at The Green Door because "It's fresh. And it just looks so good!"

This link between 'fresh' and the aesthetic appeal of the food exemplifies Bourdieu's work on "aesthetic disposition." Johnston \& Baumann describe "aesthetic disposition" as the attitude of "approaching everyday objects, like food, in a way that deemphasizes functional utility, and instead views everyday objects through an aesthetic lens of cultural appreciation and knowledge acquisition" and argue that this concept is an important component of contemporary status display (Johnston \& Baumann, 2010: 57- 
58). My data indicate that patrons of The Green Door engage with food through this lens; my interviewees minimized the functional value of food (i.e. food as simply a source of caloric energy), and instead demonstrated that they appreciated the visual appeal and cultural value of the food at The Green Door. My research participants indicated that it was these aesthetic qualities that distinguish food as 'good' and grant the individual high status as a discerning eater. Of course, the underlying sentiment is that only certain individuals truly appreciate and understand aesthetic "quality" and have "good taste" (42). This reveals the implicitly exclusive, hierarchical reality that operates beneath the seemingly inclusive, egalitarian presentation of eating 'fresh' at The Green

Door.

\section{Meeting Standards of Cleanliness \& Comfort}

Despite their insistence that the 'grass-roots' feel of The Green Door is an asset, a number of participants expressed relief that the restaurant keeps up to their personal standards of quality and cleanliness. Helen demonstrated the importance of this issue when she included an assessment of hygiene standards in her initial description of The Green Door:

High quality. Consistently good. It's fresh...I think that they're very careful in terms of hygiene. Because of course, you go to a place where you're serving yourself, we're all very aware. And they control - they always have hairnets or gloves, and you know, they're clearly following hygiene standards. And I find the customers overall, they're careful as well. You know, I never see hair in food for instance. So I feel confident that what we're consuming is hygienically ...You know within the standard that it needs to be. 
The aesthetics of the restaurant reinforce consumers' perceptions that, despite its informality, The Green Door is a high quality establishment. Tina observed:

And the atmosphere ... right? Like, I think it's very clean; it's very bright, right? Like everything is freshly painted, it's renovated; it has artwork on the walls. It's very colorful. Like I think they present themselves in a very conscious way.

Participants valued The Green Door because the fresh atmosphere is comfortable. While the casual set up and decor create an impression of accessibility and inclusiveness, participants appreciated that The Green Door maintains standards of behaviour that are sufficiently refined for an upper class clientele but not in excess:

So I feel very, very comfortable there. I'm never going to be confronted with any kind of boorish behaviour. Or you know, loud, kind of, obnoxious kind of behaviour, which makes most of us feel uncomfortable.

Viewing this 'comfort' with a more critical eye, Tina adds:

They can engage with it from a point of comfort. Right? Like, being in the Green Door and having sushi is really safe, it's really comfortable, you know, the sushi was made by a white person (with a scoff) and I think there's a lot of that going on for sure.

This passage exemplifies the tensions between authenticity/exoticism and safety/comfort that characterizes North American gourmet discourse. Amelia highlights the tenuousness of walking this line, saying that she likes the grass roots feel, but has concerns that this atmosphere reflects looser standards of cleanliness. Ultimately, she favours a more formal approach to health and safety standards:

I'm a little bit hesitant, sometimes, and I think too, what I find interesting is you know, we were talking about how the back is open. And because I see what's going on back there. And because I've noticed, like for example at The Table ${ }^{9}$

\footnotetext{
${ }^{9}$ The Table restaurant is another pay-by-weight, serve yourself vegetarian eatery in Ottawa. Like The Green Door, The Table offers a spread of vegetarian entrees, salads and desserts made daily from 'healthy,'
} 
the people, everybody is very professionally dressed who works in the back. It's ... first of all, you don't see it. So, you know, you don't see what's going on. But they dress in professional chef's clothing. Whereas at The Green Door, you know, they're in tank tops and scarves on their heads. And that's fine. But, in my mind I wonder, you know, is it as clean? Uh, so I associate The Table with being cleaner, than The Green Door. And that has an impact on which one I choose. So, even if I were ... if I were to live in the middle, I probably would frequent The Table more often now that.... I've been there. To be totally honest!

Erin shared the view that the grassroots style of The Green Door could be 'cleaned up' to showcase the success of the restaurant:

I mean it's weird. Because it is like a cafeteria-style. I mean you get your own food and sit down at very simple tables. Um, so in that respect I think it's a little ...it's pretentious in its non-pretentiousness. Because it can clearly afford better décor - better tables, better chairs. But it wants to seem like it's like a grass roots place. So it has the cafeteria style, which is fine. That's part of what I like about it. The buffet. I think it's bare-bones décor is a little ... hmmfph (skeptical huff). For me. Do you know what I mean? It's like, it's a little like, OK, you're obviously doing well, people visit here love the food. Why wouldn't you just dress up the place a little.

A number of other participants noted the behavior of the clientele often contradicts The Green Door's efforts to create an impression of inclusiveness. These research participants expressed disapproval that many patrons do not participate in the more communal activities, leaving dirty dishes on their tables rather than cleaning up after themselves, and ignoring opportunities for interaction among customers. As Jennifer said:

Because they do have these big tables, you sort of get this impression that they want people to be interactive. You do get that feeling, but then it doesn't happen.

\footnotetext{
'whole' ingredient and caters to dietary restrictions by offering vegan and gluten-free options. Opened in 2000 (12 years after The Green Door), The Table is viewed as a copy of the successful Green Door model, and despite the remarkable similarities between them, respondents often expressed a strong preference for one over the other.
} 
And that's a bit jarring! More than...you know, if you weren't expecting it. You know, you don't expect in another restaurant, for your neighbour to start talking to you ...But it seems a bit snobbish when they don't.

One participant noted that The Green Door has scaled back on design choices that were initially more radically egalitarian. Recalling an article that was written about The Green Door when it first opened, Chloe remarked:

I remember in the article there was some talk about the large communal table and how not everybody liked it. I think they've broken it up a bit, so it's smaller groups of people now.

Commenting on restaurants in Ottawa, including The Green Door, that engage with 'fresh' values, Ann said, "See, I think this is the thing with some of these places...You do get a little bit of a pretentious feel sometimes." However, Ann clarified her assessment by adding that it is not pretentious in a "high end restaurant, I have so much money way," but in more subtle indications of entitlement and privilege.

\section{Recognizing Privilege}

My data indicate that Johnston and Baumann's conceptualization of 'taste' in contemporary gourmet discourse accurately describes how 'fresh' operates within The Green Door space; individuals engage with the notion of fresh (like those of authenticity and exoticism) in an omnivorous appreciation of food which appears to work within an anti-elitist, democratic ideology, but which actually perpetuates high status ideals of 'quality,' cleanliness, and comfort that legitimize the dining experience and reflect 'good taste.' While Johnston and Baumann provide an immensely useful theoretical framework, I argue that their analysis does not address a dimension of contemporary 
North American food consumption that critically affects this dynamic. The tension between the overt performance of democratic ideals and the covert signification of discriminating tastes revealed by the Green Door patrons I interviewed suggest this process is complicated by individuals' own self-conscious recognition of privilege. My interviewees did not simply celebrate The Green Door's seemingly egalitarian atmosphere or revel in the distinction of making 'good' food choices. Rather, they demonstrated that they think critically about these dynamics and repeatedly commented on the contradictory complexity of a food system that conflates ethical ideals around health, social justice, and environmentalism with high status notions of 'good taste.'

In this section, I will illustrate how the individuals I interviewed articulated a distinct awareness of this tension between democracy and distinction. I will then explore the implications of this added complexity for one's perception of self as a food consumer. Specifically, I suggest that my interviewees' acknowledgment of privilege complicates their reflexive attempts to present a responsible, healthful, environmentally conscious, 'ethical' identity. Engaging with 'fresh' within this framework is meaningful not only in terms of how this concept obscures and shapes class inequality, but also in terms of how it is implicated in processes of self-surveillance and self-improvement (heavily promoted by modern neo-liberal ideology) that constantly threaten individuals' perceptions of selfworth. In Chapter 4, I argued that my research participants engage in highly reflexive decision making processes about food consumption that require continual research and reflect deeply internalized personal responsibility around health and wellbeing. In the 
final section of this chapter, I look specifically at how their recognition of privilege and inequality complicates these processes of 'self.' I argue that the effect of this tension on processes of 'self' - a dynamic that was not acknowledged or explored in Johnston and Baumann's work - constitutes a significant element of contemporary urban food consumption that must be understood as an important component of culture and taste.

As indicated in a number of the quotes in the preceding section, my research participants acknowledged the fact that their general approach to food and their practice of dining at The Green Door, necessitates a requisite level of privilege. In a continuation of this acknowledgement, Chloe admitted:

[The Green Door] is mostly for the privileged, you know, relatively privileged people who have money and it's, because it's convenient they are searching out these smaller places.

Chloe acknowledges that 'fresh' food is not only exclusive in that it is more expensive, but also insofar as 'fresh,' independently-owned restaurants like The Green Door are situated in more affluent neighbourhoods for the convenience of those who can afford it. Chloe noted the discrepancy in access to 'fresh' food between families who can only afford to buy homes "in the burbs," and "professionals who live in the downtown core," where they have access to Farmers' markets, independently-owned alternative grocery stores, and restaurants such as The Green Door. Amelia also noted that if you do not live in the area and do not have a car, getting to The Green Door is not easy. Now that she lives in an apartment in Hintonburg (an area of Ottawa that is more affordable to graduate students than Old Ottawa South), Amelia goes to The Green Door less than she used to, "because it is quite..., it would be two buses. No, it would be three buses to get to The 
Green Door from where I am." In contrast, while her graduate student income requires her to rely on public transit and does not permit her to live in one of Ottawa's more affluent neighbourhoods, Amelia noted that her higher level of education constitutes a form of privilege that played an important role in creating her desire to go to restaurants like The Green Door. In this way, my participants recognized they are privileged to choose 'fresh' food not only because it is viable for them to do so financially, but also because they can access a privileged knowledge around health and nutrition.

A number of participants in my study made this link between affluence, education, and health. For example, Erin noted, "wealthier people tend to be a little bit more educated and they also tend to be more, be able to afford food to take care of themselves better." According to Erin, socio-economic factors play a pivotal role in determining levels of education and this, in turn, fundamentally affects both consumers' understandings about health and nutrition, the amount of time they can devote to practices of 'self-care' or 'self-betterment,' and, ultimately, their ability to make particular food choices.

A number of my interviewees credited the restaurant's overall success to its proximity to St. Paul's University - located right across the street from The Green Door which provides the restaurant with a constant influx of highly educated clientele. Making this link, Erin observed: "[The Green Door] is also catering to a specific ... um, market, of people who are aware." Thus, the fact that the 'good' fresh food at The Green Door is only a viable solution (to the ills of our food system) for a limited group of affluent, 
educated individuals was not lost on any of my interviewees. For example, Erin commented on the tension between equality of access and distinction at The Green Door numerous times throughout the interview. In one instance she said, with a cynical smile, "I just think its image projection of being the grass roots place, in the type of neighbourhood that it's in, seems a little.... Mismatched."

Recognizing that the sale of 'fresh' food - at restaurants like The Green Door - is inextricably implicated in capitalist structures that reinforce class-based divisions of access, my research participants felt compelled to qualify their involvement in this mode of consumption. Their 'need' to adhere to a 'fresh' ethic as a means of mitigating the various types of risk outlined earlier in the thesis must be balanced against their awareness of the intrinsic inequalities that permit them access to a type of nutritional and ethical privilege that is inaccessible to so many others. Through a process of focused coding, I identified two narrative tactics that served to help them in this balancing process and to qualify their conceptualization of self as privileged. I labeled these tactics as: (1) creating distance; and (2) expressing gratitude. I argue that these tactics reflect my participants' uneasiness with their role as consumer-citizen. They understand that this role, which encourages 'ethical choices' through 'fresh' consumerism, does not ultimately allow them to address the fundamentally unethical nature of the larger food system, and therefore threatens their presentation of a healthy, ethical self as food consumers.

One of the strategies used by participants to navigate this tension was to distance themselves from their position of privilege. For example, Helen admitted that her 
husband shops at particular retailers (and avoids others) in order to maintain his identity as a money-wise immigrant:

And the reason he shops there is, um, he says 'it's the store for immigrants' and he's an immigrant himself and feels that it's just the best value and it has the broadest um, variety, and it's good quality...He says, you know, Loblaws is for, you know, the wealthy people. (Laughing) And he also sometimes goes to the Czech delicatessen to get some specialty meats.

While Helen's husband "sometimes" goes to specialty shops to get meats, and can afford to shop at Loblaws, it is important to him that he not be identified as wealthy. Many of my interviewees qualified their responses in similar ways using phrases such as, "We really don't have very much money, but..." or "I know its expensive to eat this way, but we save in other areas." Thus, Chloe clarified her tactic of distancing self by noting that The Green Door is expensive, so she chooses it as a special treat: "It makes it kind of pricey for a lunch. But if I haven't eaten out a lot, it's not a splurge that I mind so much." In this way, eating 'fresh' is presented as sufficiently important to health and wellbeing to merit the financial 'splurge' demanded from eating at The Green Door.

My research participants did not, however, want their adherence to 'fresh' to be taken as bragging, or to reflect affluence. As a case in point, one participant said explicitly that he goes to The Green Door because the grassroots, communal atmosphere minimizes his feelings of privilege:

Most restaurants I usually quickly get a favourite table, but no, there's no...um... I, I usually go [to The Green Door].... so I really don't have that, uh, that privilege, or ...yes, luxury. That's the word I'm looking for. I'd have to stand around waiting for quite some time to get a specific table. Um, but I actually, I enjoy the fact that I go in there and it is so vibrant an environment that I do have to be constantly moving around. Also ... I find that having favorite habits shuts your 
brain down. And whenever you realize you have a favourite habit, the next time you do it, you should do it completely different. Just to shake your brain up. Otherwise you become very stayed. And that ages you. You become very old very quickly.

This interviewee insisted that being pampered in up-scale dining establishments (which he has clearly had occasion to experience) is "not for [him]" because it does not challenge him to grow and improve. From his perspective, the casual, sometimes chaotic atmosphere of The Green Door not only minimizes any airs of privilege, but also functions as an exercise of self-improvement (i.e. improving brain function and mental agility).

Oftentimes interviewees used humour to distance themselves from any image of pretentiousness that could be associated with their food values. Both Tina and Jon joked that, although they tried to eat good, fresh food every day, they ate considerably more of it when they knew their parents would pick up the bill. Erin and I also developed a running joke over the course of our interview, where she would tack on a cheeky "If only I was rich!" as an addendum to comments about the food she views as 'good' and, more often than not, chooses to buy.

Participants also handled their position of privilege by expressing gratitude for their current social status. Commenting on the privilege of being able to live comfortably on a single income, Helen said, "I'm very lucky 'cause I have a husband that does all the food preparation, the cooking, etcetera at home. So we eat very wholesome." Tina expressed a similar gratitude towards her partner, who has done all of the food-related work (from gardening to grocery shopping to cooking) since she went back to school. 
Tina admitted that her ability to sustain 'fresh' food practices must be credited in large part to the efforts of a vegetable-farmer partner who shares her 'fresh' ethic. Later in the interview, Helen commented on the fact that she owes a debt of gratitude to her mother for serving fresh food to her and her siblings:

And she would prepare really good food. And so that's been kind of instilled in us. And we were really lucky, because unlike a lot of my peers - they ate out of cans, I mean, all the time, it seems - we never did. We ate real food.

Helen's comment reflects her belief that although eating 'fresh' food is an important component of healthy development and an invaluable life skill, it is also a privilege that many people do not enjoy. In addition to directing gratitude towards particular people in their lives, a number of interviewees expressed a broader, more symbolic gratitude for the privilege of being able to maintain their identity as ethical eaters. For example, in speaking about her time in Sierra Leone, Jennifer acknowledged that there are many places in the world where her vegetarian ethic would simply not be possible. Contrasting the abundance that characterizes our North American food system with the scarcity she experienced in Sierra Leone, she expressed gratitude for being able to make this purely preferential lifestyle choice.

In a similar fashion, Robert described rituals around food preparation and consumption that allow him to acknowledge the gift of 'fresh' food:

Qua Nyin. Qua Nyin, yeah.... she's the Goddess of caring, of love, nurturing, of beauty. $\mathrm{Um}$, as a corollary of dance, of performance, all things that are the essence of feminine. And we put her in our kitchen right where we store our fruits and vegetables, um, and bless our food in the morning. I also, whenever I'm going to eat my food, I take a moment to bless it. Because it's going to become part of me. 
So, um, I want whatever is going to be part of me to be full of blessings, gratitude and love.

By expressing gratitude in this way, my research participants articulate a sense of humility around their 'ethical' food behaviour that minimizes their privileged status and signifies a persona of genuine 'goodness' coherent with their perception of self.

Specifically, Robert's comment highlights the significance of food choices in the 'project of the self;' the food we choose becomes the 'self' we present to the world.

My research participants' narratives about 'fresh' food practices were characterized by a self-conscious recognition of privilege. I argue that these efforts to qualify participation in privileged modes of consumption - creating distance and expressing gratitude - complicate Johnston and Baumann's model. Johnston and Baumann's analysis of food discourse encountered in conversations with foodies indicated that food is approached primarily in apolitical terms of sensual gratification; food is appealing when it is "delicious, interesting, and worthy of eating" and social justice issues like poverty and food security are marginalized, if they are addressed at all (169). In the following section I will illustrate that, unlike Johnston and Baumann's subjects of study, my participants expressed an understanding of the complexities of contemporary food politics and made links to the broader social and environmental implications of their personal food practices. I argue that this recognition of inequality constitutes an important challenge for the modern urban individual, who engages in reflexive self-governing to ensure that they present a responsible, valued self, while continuing to participate in a food system that they recognize to be ethically problematic. 
I see that individuals' reflexive efforts are undermined by market mechanisms that promise 'ethical consumerism' while ultimately failing to address the systemic inefficiencies and inequalities of our contemporary Canadian food system.

\section{Recognizing Problems: With Privilege, Comes Responsibility}

Johnston and Baumann argue that issues of social inequality and class are either trivialized or ignored in a contemporary gourmet discourse that fails to address how food practices "relate to larger structures of class and material privilege" (Johnston \& Baumann, 2010: 59). I found, however, that my research participants' efforts to distance themselves from privilege were not mere attempts to obscure or deny their privileged status. Rather, they reflect participants' uneasiness, or guilt, about participating in a consumer culture that satisfies individual 'ethics,' but fails to address the fundamentally unethical processes and practices of the larger food system of which they are a part. I argue that this dilemma constitutes a significant dynamic in contemporary food consumption because it threatens individuals' ability to present a responsible self.

All of my interviewees demonstrated an awareness of the inherent inequalities of our contemporary Canadian food system and expressed genuine concern about the impacts of these injustices. Repeatedly using words like "worry," "worrisome," and "troubling," Helen outlines some of the key issues that were articulated by interviewees:

I worry about inequities. Like, grotesque inequities in food consumption around the world. In what's available to us, and what's available to other people in the world. Um, where we are clearly over-consuming. I find that troubling. That we are - there's a disproportionate quantity of everything. Available to us, here. And, we know, in other parts of the world. That's troubling. That's 
philosophical, these are ethical questions, but they're, they're huge. And they're very troubling. Um....yeah, so, so the inequities I worry I about. I worry about the contamination, chemicals, hormones, uh, also, also, uh, you know when you hear that people are getting these terrible diseases from eating certain like, it was beef for a while. That is worrisome. That's very worrisome. I worry about the laborers who are producing a lot of our food, including coffee. Um, that, they are not being paid fair wages and they are not, they're not given proper conditions to work it. Actually that worries me a lot.

Through responses such as this one, my research participants indicated that they $d o$ understand how their food consumption practices "relate to larger structures of class and material privilege" (Johnston \& Baumann, 2010: 59). Specifically, respondents demonstrated that they recognize the broader social dynamics that affect who is able to take up these issues and who is not. While all respondents clearly articulated their belief that they need to 'take responsibility' for their choices, they generally shared the belief that larger societal structures, rather than the individual, are "to blame" for the poor dietary choices that are made by others. Speaking with compassion, rather than judgment, Helen described her dismay at the lunches she saw children eating in her role as an elementary school principal:

And many families, the majority for very good reasons, they're just too swamped by other responsibilities, including survival, you know, jobs and what have you, who can't participate more actively. But, I, I'm dismayed at the number of children that I see with uh, lunchables in their lunch. You know those packages with ugghh; it's very, very upsetting. Um, with, you know, with parents rushing in at the last minute to bring them stuff from McDonalds or Timmy's or what have you because they can't get organized, so they just give this to their children. Chips, uh, like, junk food basically.

A number of my research participants were clear that they do not want to be mistaken for one of those 'foodies' who are not concerned with social justice and environmental 
activism. For instance, when I asked Tina if she considered herself a 'foodie,' she offered this response:

Ummm...a lot of the race and class issues don't really get taken up or negotiated with the foodie discourse. Or like the emphasis on taste.... stupid word authenticity, really get prioritized above considerations of you know, where did the food come from, under what conditions, and those types of more ... kind of ... political economy analysis of it. Ummm... so I could be a critically minded foodie...but...

More than simply expressing an awareness of widely popularized concerns such as Mad Cow Disease or diabetes, my research participants indicated that they "think critically and reflexively about their participation in food culture" (Johnston \& Baumann, 2010:

59). Interviewees demonstrated they were informed on key food consumption problems and exhibited a belief in the importance of interrogating potential solutions, to identify how progressive sentiments may be complicated in practice or appropriated by corporate interests. Tina described her investigative process in the following manner:

It was sort of a broader investigation of where my food comes from, and you know, I got curious about things like organics, and the fair trade movement. And sort of thinking critically about, say, capitalism in general, and like how that relates to specifically to like, agriculture business and how we get our food, and what are the environmental impacts, but also specifically, what are like, the human impacts. So like, from a labour perspective I was really interested. Like, migrant labour, so, even if we're talking about local food, a lot of local farmers using dozens of Mexican migrant labourers and treat them like shit. So, do you, how does that complicate our notions of local. Or, same with organics, right. If it's Loblaws organics... So, probably for the past 7 years it's been kind of ...I'll get really excited about an idea. Yeah, and then slowly, being more and more critical about it. Like at first, being like, 'yeah! Free Trade'! Yes! And then being like, OK, maybe fair trade in and of itself isn't enough and same with organics. Just saying organic isn't enough. 
Interviewees acknowledged that many of their values have been co-opted in popular fads. This process of co-optation placed them in a problematic position. To address this problem, some individuals adopted an "it's got to start somewhere" stance arguing, for example, that the appearance of an Organics line in Loblaws or Walmart may allow the 'organic movement' to "gain traction and exposure in the mainstream." Others argued that these 'fresh' trends served only as status-based marketing strategies that capitalize on 'good' food practices. All participants acknowledged, however, that this process represents a contentious issue and, regardless of which position they took in the debate, their participation as consumers, in this corporatization of 'fresh' was the only viable option if they were going to choose good, 'fresh' food. However, despite the fact that their food values have been co-opted by corporate players who recognize the social currency of 'fresh', my research participants did not lose their concern about fair wages or eating chemical-free foods. Caught up in these anxious contradictions, they still attempt to "make the correct choices" - i.e. choose 'fresh' - however they can.

Johnston and Baumann criticize the logic of this 'ethical consumption frame' (166), wherein suppliers present 'ethical,' 'fresh' commodities as win-win solutions that are 'good for business, good for you!' Similar to Johnston and Baumann, my research participants indicate that this framing process builds on a deeply problematic "neo-liberal vision of environmental and social sustainability" that relies on market mechanisms to provide the requisite systemic change (169). This argument is particularly relevant to my thesis because of how it positions the food consumer. Although Johnston and Baumann do not explore the effects of this trend on the individual consumer in any great depth, 
they observe: "When faced with the plethora of political concerns that surround our food [in a commodity-based system] - and the feelings of guilt that accompany them - the proposed "solution" is to become an informed consumer" $(170)$. This system relies on "enlightened, educated consumers' self-interest as the primary motor for social change" (170). Based on the accounts of my research participants, I propose that, despite tireless efforts to become an informed - i.e. responsible - consumer, individuals are ultimately unable to come to terms with their participation in a system that is inaccessible to a majority and reproduces inequality. These participants consistently expressed their belief that, in spite of their concerted efforts to sustain self as an ethical food consumer, they were, ultimately, unsuccessful. Thus, for example, having just outlined a number of the "ethically abhorrent practices" that characterize of our contemporary Canadian food system (such as factory farms), Helen admits that she continues participating in the system that perpetuates these injustices, and describes a sentiment of ethical defeat when she says,

I am very aware of the whole conundrum. Uh, and I understand that it is actually quite pathetic given everything we understand about the needs of food in the world, but we're still doing this. And in that sense, I probably think vegetarianism is probably the most responsible um, way to be eating. And probably um, uh, like, ethically the right thing to be doing. Uh, that more people haven't gone that route, it's perplexing, but I haven't either.

In less dramatic terms, interviewees described the "imperfections" of their dietary practices (such as eating highly-processed potato chips) with regret, saying they "should know better." These types of 'bad,' 'unethical' food consumption practices were perceived by my interviewees as threatening to erode the moral fabric of an individual's 
identity, while good, 'fresh' food consumption practices granted individuals assurance that they have successfully constructed a responsible self in terms of one's food consumption. Attempting to frame his approach to food practices in forgiving, nonjudgmental terms, Robert highlights the potency of food consumption as an endlessly improvable variable in the project of the self:

Well, there's always that temptation to judgment. Of 'did they go far enough?' And what I want to see is that you're making the effort and that you're constantly, like you're bettering the effort.

Erin links this project of self-betterment specifically to patrons of The Green Door:

It's usually situations where there are people who are clearly invested in a particular lifestyle. An identity, which is associated with something like The Green Door. And they're usually talking about things like self-betterment, and all of these things. But they can be very militant about it! (Laughing) And a couple of times it was basically people, really trying to convince, let's say another person, about their lifestyle. And their way of existing. And you'd think they'd be relaxed about it (laughing)! And they're not!

As Erin illustrates in this response, stakes are high when considering individual efforts to maintain an ethical, responsible self as a food consumer. These practices can require 'militant' attention and are often characterized by high levels of stress that contrast and, at times, undermine the positive intention of creating a healthier, more ethical food practice.

Interestingly, I found that this dynamic was evident in the majority of my interviews. My research participants demonstrated high levels of enthusiasm and passion as they talked about the discoveries they have made in this deeply personal aspect of their lives. While some participants engaged in extremely complex, articulate analyses of food issues in our interview, discussing these issues was never simply an intellectual exercise. 
Despite the innocuous nature of my research questions (See Appendix B), a number of my participants got notably emotional as they discussed the impact 'fresh' food practices have on their life and the implications of our food system on society at large. For example, near the end of our interview, Helen began to tear up and remarked:

I can get kind of emotional about it, but, 'cause it's very, it's very troubling, that in a world like ours, where, I bet you everyone in this place [Bridgehead] ${ }^{10}$ is educated, and this is the best we've come up with so far. I think we can be doing a lot better.

In this way, Helen asserts the general perspective offered by my research participants regarding how the failure of the individual to make ethical choices is compounded by his/her awareness of the ethical issues that trouble our food system. Additionally, these participants repeatedly indicated that because of their privilege (whether it be in terms of access to financial or knowledge resources) they should be able to 'do better.' They agreed that with privilege, comes responsibility, and, having accepted this responsibility, they set out to make 'better' choices. As such, The Green Door fulfilled their need for a safe space where the requirement of balancing these choices were minimized and, in the majority of cases, removed altogether.

\section{"Feeling Better" by Entering Through The Green Door}

The individuals I spoke with indicated that The Green Door Restaurant is a particularly desirable dining establishment because it offers them the opportunity to make 'ethical'

\footnotetext{
${ }^{10}$ Bridgehead is a popular locally owned coffee shop chain in Ottawa that serves "fairly traded organic, and shade grown coffees from small-scale farmers," as well as a menu of freshly made soups, sandwiches, salads, and pastries. There are now 10 Bridgehead locations across Ottawa.
} 
food choices that reinforce their identity as a 'responsible' food consumer. Specifically, The Green Door provides a space for maintaining a more positive sense of self. As Helen noted, "[The Green Door] is a hopeful business. It's a business that is modeling a better way to be."

Research participants repeatedly shared the sentiment with me that The Green Door does the 'ethical' work for you. Robert said:

Yes, I think they're aware of food systems, and they are reflective upon their impact on the food systems. Possibly not as reflective as other restaurants that we've gone to, but I think that they might be the most reflective in Ottawa.

Because The Green Door engages in a reflexive ethical interrogation of their food, research participants can simply relax and enjoy their meal without worrying about doing the work themselves to maintain their positive sense of self as ethical eaters.

A number of participants articulated that they could take a break from their dietary vigilance at The Green Door because it is a "safe place." Renee declared: "Yeah, actually, that's really what it is...It's a safe place in terms of the people who are there. It's a safe place in terms of the food that's on offer." Participants felt that The Green Door is a particularly attractive option because all of the food that they offer is ethical in some sense; the patron does not need to worry about making a 'wrong move.' Renee also indicated that The Green Door is a 'safe' space socially, insofar as the other patrons who will dine there share a common food 'ethic.' In this way, the patronage at The Green Door gives them social support for self as part of a larger group of ethical food consumers. 
Tina observed, however, that beneath the surface of this shared, overarching 'fresh' ethic, the specifics of each diner's personal food practice are extremely individualized. She sees that the 'fresh' space provided at The Green Door offers patrons the opportunity to attend to their individualized food ethics:

I think they've done a really good job of like, kind of like, providing this space that's open to interpretations... So some people go because it's just really good food.... And some people go because it's organic and they have lots of gluten-free options. Or ... Um... they've done a ... like it's an interesting intersection into lots of different ethics or politics that people bring to food. Um, yeah, I don't know, so I think ... it provides a lot of different, like it provides a wide opportunity for people to engage in their food in different ways.

Tina accepts the notion that she, like any other Green Door patron, is participating in food practices at The Green Door as an individual, who makes choices that best reflect the food values that are important to her. In this passage, she illustrates the link between a consumer's individualistic efforts to achieve a responsible self, and the success of The Green Door restaurant. Because the multiple elements of the 'fresh food ethic' emerge, interact, take focus, or recede as priorities for individual consumers, food retailers are challenged with the task of offering consumers a product, or in the case of a restaurant, an experience, that accommodates, or better epitomizes, these values. The Green Door models a solution: create a dining experience that is 'fresh.'

In the urban center, the hunger for 'freshness' invariably translates into an increasing niche market of consumers who want 'freshness' fast. My participants valued The Green Door because it makes 'ethical' eating convenient for them. For example, Amelia admits that she likes The Green Door because she can satisfy her food ethic quickly whenever she wants. She concluded: "That's why I love them so much.... it's 
almost like my fast food. But it's really healthy." The implicit contradiction of this statement is revealing. While participants want to re-connect with the 'natural state' of their food, the realities of doing so (for example, tending a garden and witnessing vegetables mature slowly over several months or making a meal from scratch) are not often compatible with the fast pace of modern urban life. 'Ethical' consumption then becomes about finding short cuts that generate the same feelings of safety and ethical assurance. For my research participants, The Green Door represents a viable alternative.

My research participants noted that whether or not they are 'doing' better by going to The Green Door (i.e. inciting social change), they feel better when they choose to eat at a restaurant that is committed to 'ethical' practices. Helen recognized that part of the value of The Green Door is how it makes consumers feel:

We probably all feel better when we go there, because we feel that we're at least partially contributing to a better way of being in the world. Um, which could, um, impact, uh, on the whole world, because it's um ... there's not enough Green Doors!

Helen's declaration at the end of this passage opens up an important question. Do we need more Green Doors? Is this the solution to the ethical (i.e. social, environmental, physical) shortcomings of our contemporary Canadian food system? Could this "impact the whole world"'? According to all appearances, The Green Door is 'win-win.' Not only does this restaurant understand the issues and make 'ethical' choices, it also provides a culinary experience that patrons consistently, and enthusiastically enjoy. And yet, I would propose with resounding clarity that the answer to these questions is invariably, no. To elucidate this position, I go back to the origin of this analysis. 
Johnston and Baumann (2010) observe that the tension between democracy and distinction that characterizes contemporary food discourse effectively conflates, rather than illuminates, fundamental inequities in class and material privilege. They argue that issues of social inequality and class become either trivialized or ignored as individuals engage in food consumption practices that only seem to reflect a more egalitarian approach to food. Johnston and Bauman offer a relevant critique of contemporary food consumption practices. However, I suggest that there are significant implications on the individual that are not addressed in this work. My data indicates that although 'fresh' operates within the ideological tension between democracy and distinction described by these authors, those individuals who adopt a fresh food ethic are strongly aware of how their participation in certain food practices "relates to larger structures of class and material privilege" (Johnston \& Baumann, 2010: 59). The participants in my study recognize that their ability to act on their 'fresh' food values reflects a position of privilege. This recognition adds a layer of complexity that is significant for the analysis of food consumption because it complicates the individual's ability to present a 'responsible,' 'ethical' self. Specifically, in a system that relies on class inequalities and externalized environmental costs, the failure of the individual to present a sufficiently 'responsible,' 'ethical' self (and the self-criticism and guilt that accompanies it) is predetermined and inevitable due to those very costs and class inequalities.

While the significance of this dynamic on processes of 'self' is considerable, my data analysis also indicates a broader failure of the modern neo-liberal ideology of 
individualism that supports Western culture. This study indicates that the responsibilization of the individual as consumer-citizen maintained by that ideology has damaging consequences on the individual at the same time as it ensures that the systemic problems of our industrialized food system will not be addressed. As long as individuals are pre-occupied with the minute successes and inevitable failures of their personal 'project of the self' as ethical food consumers, they cannot be engaged meaningfully in collective action designed to change the larger system of food production. Despite the personal appeal of prescribing more Green Doors as an antidote for this problem, the food practices that take place within this 'fresh' space must ultimately be understood as a reflection of a modern, neo-liberal paradigm that engages the individual in an endless project of self-improvement; one that effectively distracts from and displaces genuine individual efforts for real change.

In my concluding chapter, I outline the key arguments made in this thesis and expand further on how these findings highlight the broader limitations of an individualistic, consumer-based activism. Appreciating the merits of 'fresh' as a response to the industrialization of our food system, I argue that we need to shift the scope of the 'fresh food ethic' from the individual to the collective in order to address fundamental issues of inequality and sustainability and to meet shared goals of more ethical food consumption practices. 


\section{CHAPTER 6}

\section{CONCLUSION: Final Thoughts on 'Fresh'}

This thesis has been concerned with identifying the values that inform food choice. Current processes of industrialization and globalization have dramatically changed the scope of possibility of food selection for the individual consumer. Within this contemporary urban food system, individuals are constantly enlisted to make choices, from an immense variety of options, about the food they eat. I propose that this situation constitutes a particularly significant dynamic in contemporary society, and have chosen to explore it as the primary focus of my Master's thesis. In this final chapter, I offer my personal interpretation of my analysis, outlining what I believe to be the implications of my findings and arguing for a collective, rather than individualistic, food activism.

Whereas I have been meticulous, up to this point, about letting my data guide and illustrate my analysis, I now present a personal reflection that reveals my position in the research and my impressions of its broader significance. I propose that interrogating contemporary food values is an immensely relevant and important task, which requires the creative thinking of scholars who will work enthusiastically on developing deeper understandings of, and innovative solutions for, Western urban food consumption. It is my hope that my work can serve as a contribution in these efforts.

I begin this section by reviewing the core components of my analysis. Focusing my research on urban food choice at The Green Door Restaurant, I have investigated the values that certain individuals bring to their food consumption practices, and explored 
how food choices are manifested in the action of dining out. Through a detailed analysis of my research data, I identified 'fresh' as a core value of urban food consumption for a particular group of people and have engaged in a thorough examination of how this value operates within the context of a contemporary Canadian food system. Recognizing the prevalence of 'fresh' in my data, I identified five component layers of 'freshness' and illustrated how each of these sub-values - local, seasonal, non-contaminated, nonpackaged, and human handled - represents a shared concern with consuming food that is 'as close to its natural state as possible.' The participants in my study engage with these values in order to mitigate the health risks that they perceive to have occurred from an increasingly industrialized food system. Because they cannot successfully attend to each of these values all the time, my research participants can rely on a broad, overarching commitment to "freshness" to construct a personal food practice that is 'good enough.' I argue that this commitment is significant because it exemplifies the increasing responsibilization of the individual in the modern neo-liberal era. Specifically, when considering food consumption, the individual is tasked with being an informed, responsible consumer and making the 'right' choices to ensure optimal standards of health and wellbeing.

My research indicates that The Green Door restaurant engages with the values of the 'fresh food ethic' in distinct and varied ways to create a dining experience that attracts the types of food consumers represented by my research participants. Employing Johnston and Baumann's (2010) model, I illustrate that 'fresh' invokes democratic, egalitarian values of accessibility and inclusiveness, while also functioning covertly as a 
marker of status and distinction. While Johnston and Baumann conclude that this dichotomy effectively eliminates considerations of class and inequality in contemporary gourmet discourse, I found that patrons of The Green Door demonstrate an awareness of privilege that complicates this analysis. Given this awareness, my research participants experienced a tension that disrupted their efforts to present an ethical, responsible self as a food consumer and required them to engage in constant self-reflection and concerted food consumption activities. I argue that these types of self-regulation practices constitute a critical breakdown in processes of self for the 'ethical' individual, who recognizes the fundamentally unethical nature of the larger food production system of which he or she is a part. It also points to the limitations of market-based, consumerchoice solutions to the environmental and social problems that afflict our contemporary Canadian foodscape.

\section{Connecting 'Fresh' to Collective Action}

Once I identified 'fresh' as a core category in my interview data and started to really think about what it means, I have come to find it everywhere. Subway invites us to "Eat Fresh;" Wendy's distinguishes its burger patties as “Fresh, never Frozen;” A\&P grocery chain is "Fresh Obsessed." Notions of 'fresh' pervade contemporary marketing campaigns, and it is no wonder why. Freidberg (2009) notes, "these days we are constantly reminded that freshness is good for us" (5). With recommendations from the Federal Ministry of Health urging consumers to shop around the perimeter of the grocery store and to avoid high sugar, high fat packaged foods, media reports citing links between 
pesticide consumption and cancer rates, and doctors urging patients to mitigate disease by eating an apple a day, corporations can target a readily available niche market of 'educated' individuals who have been fully briefed on these issues, and believe that 'fresh' is the responsible choice. As Freidberg (2009) suggests, "few food qualities seem as unquestionably good as freshness" (2).

While the logic of promoting food products (or restaurants) as 'fresh' is clear, my data suggest that the implications of this marketing trend are far reaching and constitute one of the most important complications of making 'good' food choices. I have illustrated that 'fresh' serves as a catchall signifier of 'good' food; it invokes antiindustrial sentiments that quell consumer fears, while maintaining a cheerful positivity that emphasizes pleasure. With these capacities, this 'value' of freshness has proven to be an immensely powerful tool in product development and food marketing. We buy our groceries from big box stores, we eat poor-quality, nutrient deficient, chemical laden burgers, and we fill our landfills with fast food wrappers, convinced that we are choosing an option that is at least "fresh" in some sense of the word. While genuinely 'fresh' food is decidedly not accessible to a growing number of Canadians (with urban food deserts and insufficient social assistance shaping the diets of the lower class), 'fresh' is presented as an easy choice that is applicable to and accessible for everyone. Conceptualizations of 'fresh' blur boundaries of 'good' and 'bad' food to make less healthful, unethical food more palatable. I suggest that the dynamic I have identified in my data, whereby individuals and businesses alike rely on a broad, ambiguous commitment to 'freshness,' exemplifies the shortcomings of consumer-based activism. While there is the occasional 
Green Door restaurant which seems to genuinely stand for connecting consumers to the 'natural state' of their food, all of my participants acknowledged that the food they enjoy in this setting is the exception, not the rule, in their lives, despite their deeply held belief that eating food that it as 'close to its natural state as possible' is fundamental to good health, symbolizes a basic stewardship of the environmental, and produces a high quality of life.

Of course, 'fresh' is not the first big idea to stimulate the appetites of environmentally conscious, 'ethical' consumers. Ten years ago, 'organic' was the industry buzz word. Similar to 'fresh,' 'organic' represented a reaction against the industrialization of food and appealed to consumer desires with ambiguous promises of naturalness and earthiness (Johnston et al, 2009). Without established regulations or certifications, corporations were free to apply the organic label to their products without any accountability about processes and practices (Johnston et al., 2009). Now that we have relatively strict regulations around organic practices, this term can no longer be applied in broad strokes to highlight the naturalness of food or quell the fears that emerge out of an industrial food system. I suggest that fresh can be understood as an evolution of the idea of organic. Still incorporating organic as one of its key tenets, the increasing prevalence of 'fresh' as a contemporary food value reflects a further step in a direction of abstraction. I see that, rather than moving forward with concrete solutions, we have simply found new language to ease consumer anxieties and frame individual choices as 'good.' 
So what is the fundamental problem? And how can it be addressed? The individuals I interviewed offered their suggestions on how to move forward; their responses are revealing. When I asked Erin what should be done, she suggested that we need to "popularize" ethical eating. She offered the example of former Montreal Canadians hockey player Georges Laraque, who is "bringing ethical consumption into the mainstream." Laraque, and the body builder referenced later in the passage, are framed as role models for 'ethical' consumption:

Yeah, he's a vegan. Yeah, and he drives, like a hybrid car, and all that kind of stuff. Like, he's pretty, like hippy-ish. (Laughing) But he's, like he's a hockey play, right? It's kind of a disconnect between people's usual ideas of macho, but he's a vegan. And um, I read in the Globe and Mail about this book, of a guy. He's a body builder, he's like one of the world's best body builders, and he's also, he's a vegetarian. And it wasn't thought possible that you could gain that much muscle mass while eating only vegetables, but he put out a book, like describing nutrition and describing, OK look, if you eat the right kind of vegetables then you can, you can, gain muscle mass. And you'll feel better at the same time.

In this passage, Erin describes precisely the individualistic mentality that is, I argue, at the core of the problem. While making the choice to disengage with the industrialized slaughter of animals, or to drive a low-carbon-footprint car indicates an awareness of important ethical and environmental issues, these choices are executed within a neoliberal paradigm that valorizes the efforts of affluent individuals who have, ostensibly, achieved a level of optimal health. Significantly, Erin's account does not contain any reference to widespread solutions or fundamental change; instead it invites other individuals to engage in practices of self-betterment that can (but almost definitely will not) earn them status, like the body builder, as the "world's best." The reader is taught to eat the 'right' kind of vegetables to achieve socially sanctioned standards of health and 
masculinity but not to question the manner in which most food is produced. I argue that this individualistic approach characterizes contemporary food discourse and is significant because it sets the rules that we play by as consumers; a set of rules that does not question or threaten the status quo.

Although Erin's response focuses on individual self-improvement, the consumer model that she describes (i.e. buying a hybrid car to be environmentally-friendly) is often presented as a viable system for social change. Within the context of a capitalist economic structure, individuals are given access to every conceivable food option and are then held responsible to make 'good' food choices. Acting as consumer-citizens, individuals are expected to 'vote with their wallet' to create the food system they want (Lang, 1998). Food is a particularly interesting commodity in this 'democratic' system, because it is relatively inexpensive and short-lasting but also a necessity; individuals can 'vote' in ways that feel manageable, multiple times a day (Lang, 1998). Viewing food choices in this way is understandably tempting - my participants can enjoy a delicious meal at The Green Door as an act of 'resistance' - but I argue that these market-based solutions obscure, rather than solve, the inequities and dangers of our contemporary urban food practices. Buying organic meat when it goes on sale, choosing fair trade coffee when it is convenient, or going to the farmers market on a Sunday morning when the weather seems nice (all practices described by my research participants) are activities of privilege; they do not address the fundamental inefficiencies and innumerable externalized costs of our industrial food system. 
Johnston et al. (2009) assert, "the contradictions and limitations of consumerbased forms of political action are increasingly well-documented, theorized, and linked to neo-liberal agendas" (Freidberg, 2004; Goodman, 2004: 909; Guthman, 2007: 263; Johnston, 2008 in Johnston et al., 2009: 525). The participants in my research study exemplified this trend, articulating specific patterns of behaviour that allow them to present self as ethical eaters; they engaged in consumption practices that are most accurately understood as efforts of self-betterment in a 'project of the self.' Importantly, these actions cannot be viewed in activist terms. Rather, such efforts effectively preoccupy the individual in a losing battle to present a responsible, 'ethical' self, and in doing, so inhibit unified social action.

I argue that we need to re-conceptualize 'ethical food practices' in terms of collective action as opposed to individual responsibility. Johnston et al. (2009) articulate this vision when they write:

Most crucially we need a collective re-thinking of the industrial food system that can, and must, motivate a collective challenge to the neo-liberal reliance on consumer choice as the optimal means of regulating how and what we eat. (528)

Instead of a false democracy based on a consumerist model of individual choice and 'voting with your wallet,' Johnston et al. propose that we engage in the "more challenging elements of democratic process, such as moving towards decentralized ownership structures and creating non-commodified social and economic relationships around food" (Johnston et al., 2009: 525). Specifically, these authors promote a "rethinking of strategies to resists the inequities associated with industrial food production and distribution" (Johnston et al., 2009: 514). Part of this vision of food 
democracy includes alternative agricultural initiatives: "Farmers' markets, community supported agriculture (CSA) and food box schemes are represented as alternatives to a corporate supermarket system that operates without consideration for resource depletion, and the inequities inherent in food production" (Johnston et al. 2009: 510). ${ }^{11}$

While these alternatives must not be viewed as simple, clear-cut solutions, and undoubtedly present challenges of their own, they do operate according to collective values. First, these alternatives represent a "decentralization of power, out of the hands of corporations and into the hands of the people" (515). Similar to Johnston et al., I believe that meaningful dialogue about collective needs cannot happen within a consumer-as-citizen paradigm that prioritizes corporate interests and obscures inequality. Secondly, these alternative food practices require individuals to create and rely on social networks as a primary support system. As Johnston et al. write, "social relationships foster democratic accountability and substantive equality, which are of primary importance to a meaningful vision of food democracy" (Johnston et al., 2009: 515). For this reason, food democracy cannot be a "product of the market" (527); rather it must be built through collective efforts that address collective needs. I see this vision of food

\footnotetext{
${ }^{11}$ Importantly, many of the patrons I interviewed participate in these types of alternative initiatives and spoke to me about their concern for creating a more sustainable food system. I did not explore my research participants' political engagement or community activism in any great detail in my interviews and therefore, cannot speak to how these actions may or may not contribute to substantive social change. However, I suggest that my interviewees' involvement in these various strategies of food activism further highlights the tension that I have identified between individuals' genuine desire to act ethically and their participation in an industrialized Western food system that is fundamentally unsustainable, but comforts consumers with promises of 'fresh.' Additional research is required to explore how individuals' involvement in alternative food production and consumption systems could constitute meaningful collective action and create a more sustainable Western food system.
} 
democracy as a particularly appropriate final destination for my research; not only would this activity result in a more equal and ethical food system, it would also effectively release the individual from arguably oppressive, and inevitably futile, efforts to present an ethical, responsible self in his or her food consumption.

\section{Why Does It Matter?}

The people that I spoke with love food. They identified themselves as 'big' eaters, 'avid' eaters, 'passionate' eaters, and 'joyful' eaters. For them, eating means indulging in one of life's absolute greatest pleasures. And yet my conversations with each of these eaters very clearly indicated that eating is a project; it requires awareness, strategy, and care. My research demonstrates that making choices about food in a contemporary Western context requires perpetual research and rigid self-surveillance; the individuals I spoke with sought out expert advice and weighed corporate messaging in an effort to present an evermore 'ethical' self. While environmental issues and concerns about inequality were very much at the fore of our conversations, the focus of our discussions remained fixed on efforts to present a 'responsible' self in one's food consumption, rather than on inciting meaningful social change.

I suggest that my interviewees' desire for 'freshness' is an entirely appropriate vision for contemporary food consumers, but we cannot achieve that vision without engaging in a fundamental re-thinking of how we engage with the food. Freidberg notes that the appeal of fresh "lies in the anxieties and dilemmas borne of industrial capitalism and the culture of mass consumption" (Freidberg, 2009: 3). If we continue to engage with food solely as individual consumers, the inequalities and environmental 
inefficiencies that threaten the health of our food system will persist, and continue to undermine individuals' genuine efforts to 'be ethical.' Alternatively, we could engage with food as a collective to challenge the corporate vision that "food is principally a commodity produced for sale" and replaces it with a humanitarian view that access to safe and nutritious food is a basic right (Johnston et al., 2009: 514). My interviewees indicated that valuing 'fresh' food is good, common sense. I argue that we now need to make the leap from common sense, to collective action. 


\section{WORKS CITED}

Appedurai, A. 1988. "How to Make a National Cuisine: Cookbooks in Contemporary India" in C. Counihan and P. Van Esterik (eds), Food and Culture: Reader $\left(2^{\text {nd }}\right.$ Edition). New York: Routledge, pp. 289-307.

Ashley, B., J. Hollows, S. Jones and B. Taylor. 2005. "Food Ethics and Anxieties." In Food and Cultural Studies. New York: Routledge. pp. 187-203.

Bauman, S. and J. Johnston. 2007. "Democracy vs. Distinction: A Study of Omnivorousness in Gourmet Food Writing" in American Journal of Sociology, 3(1): pp. 165-204.

- 2010. Foodies: Democracy and Distinction in the Gourmet Foodscape. New York: Routledge.

Beck, U. 2002. "The Cosmopolitan Society and its Enemies." Theory, Culture \& Society. 19(1-2): pg. 17-44.

Belasco, W.J. 2002. 'Food Matters: Perspectives on an Emerging Field' in Food Nations: Selling Taste in Consumer Societies, New York: Routledge.

Blumer, H. 1969. Symbolic Interactionism. Englewood Cliffs, NJ: Prentice-Hall.

Bordo, S. 1993. "Anorexia Nervosa: Psychopathology as the Crystallization of Culture" in C. Counihan and P. Van Esterik (eds), Food and Culture: A Reader $\left(2^{\text {nd }}\right.$ Edition). New York: Routledge. pp. 162-186.

Bourdieu, P. 1984. "Taste of Luxury, Taste of Necessity" in C. Korsmeyer (ed), The Taste Culture Reader: Experiencing Food and Drink. New York: Berg., pp. 7278.

- 1984. Distinction: A Social Critique of the Judgment of Taste, translated by R. Nice. Cambridge, MA: Harvard University Press.

Caplan, P. 1997. Food, Health and Identity. Routledge.

Charmaz, K. 2006. Constructing Grounded Theory: A Practical Guide Through Qualitative Analysis. London: Sage.

Doucet, A. \& Mauthner, N. 2003. "Reflexive Accounts and Accounts of Reflexivity in Qualitative Data Analysis." In Sociology. London: Sage, 37(3). Pp. 413-431

Douglas, M. 1999. "Deciphering a Meal." In C. Counihan and P. Van Esterik (eds.) Food and Culture: A Reader (2 ${ }^{\text {nd }}$ Edition). New York: Routledge, pp. 44-53 
Elias, N. 2000. The Civilizing Process. Sociogenetic and Psychogenetic Investigations. Revised edition of 1994. Oxford, Blackwell.

Emerson, R., R. Fretz, \& L. Shaw. 1995. "Participant Observation \& Fieldnotes." in Handbook of Ethnography. London: Sage. pp. 352-267

Enoch, G. \& Buchbinder, E. 2005. "The interactive construction of narrative styles in sensitive interviews: The case of domestic violence research." Qualitative Inquiry. 11, pp. 588-617.

Fawcett, B. \& Hearn, J. 2004. "Researching others: Epistemology, experience, standpoints and participation." International Journal of Social Research Methodology. 7(3). Pp. 201-218.

Feilzer, M. 2010. "Doing Mixed Methods Research Pragmatically: Implications for the Rediscovery of Pragmatism as a Research Paradigm." Journal of Mixed Methods Research. London: Sage, 4(1). Pp. 6-16.

Fine, G. 2003. "Crafting Authenticity: The Validation of Identity in Self-Taught Art." Theory and Society 33(2): pp. 153-180.

Fischler, C. 1980. Food Habits, Social Change, and the Nature/Culture Dilemma.

- 1988. "Food, Self and Identity." Social Science Information. 27(2): 275292.

- 1990. L'Homnivore: Le Gout, La Cuisine et Le Corps. Paris: Editions Odile Jacob.

Flyvbjerg, B. 2001. Making Social Science Matter: Why social inquiry fails and how it can succeed again. New York: Cambridge University Press.

Foucault, M. 1978. The History of Sexuality: Vol. I: An introduction. Vintage Books, New York.

Freidberg, S. 2009. Fresh: A Perishable History. London: The Belknap Press of Harvard University Press.

Fusco. 2006. "Inscribing healthification: governance, risk, surveillance and the subjects and spaces of fitness and health." Health \& Place. 12(1), pp. 65-78.

Giddens, A. 1991. Modernity \& Self Identity: Self and Society in the Late Modern Age. Cambridge: Polity Press. 
Glaser, BG. 1978. Theoretical Sensitivity: Advances in the methodology of Grounded Theory. Sociology Press.

Glaser, BG. \& A. Strauss. 1967. Discovery of Grounded Theory. Strategies for Qualitative Research. Sociology Press.

Goffman, Erving. 1959. The Presentation of Self In Everyday Life. New York: Doubleday Anchor Books.

Guide To The Green Door. 2010. www.thegreendoor.ca.

Guthman, J. 2003. "Fast food/organic food: reflexive tastes and the making of 'yuppie chow"' in Social and Cultural Geography, 4(1): pp. 45-58.

Haden, R. 2005. "Taste in an Age of Convenience: From Frozen Food to Meal in 'the Matrix"' in C. Korsmeyer (ed.) The Taste Culture Reader: Experiencing Food and Drink. New York: Berg. pp. 344-358.

Johnston, J. A. Biro and N. Mackendrick. 2009. "Lost in the Supermarket: The Corporate Organic Foodscape and the Struggle for Food Democracy" in Antipode: A Radical Journal of Geography.

Lang, T. 1998. "Toward a Food Democracy" in S. Griffiths and J. Wallace (eds.), Consuming Passions: Food in an Age of Anxiety. New York: Manchester University Press, pp. 13-23.

Lofland, J. \& L. Lofland. 1984. Analyzing social settings: A guide to qualitative observation and analysis. Belmont, CA: Wadsworth.

- 1995. Analyzing Social Settings: A Guide to Qualitative Observation and Analysis. Belmont, CA: Wadsworth.

Long, L. 2004. "Culinary Tourism: A Folkloristic Perspective on Eating and Otherness" in L. Long (ed.), Culinary Tourism. Lexington: The University Press of Kentucky, pp. $20-49$.

Lupton, D. 1995. The Imperative of Health: Public Health and the Regulated Body, Sage, London.

- 1996. "Food, Health, and Nature" in Food, the Body and the Self. London: Sage Publications. Pp. 68-93.

- 1996. "Theoretical Perspectives on Food and Eating" in Food, the Body and the Self. London: Sage Publications, pp. 6-36 (Chapter 1). 
- 1999. Risk. London: Routledge.

Lupton, D. \& Peterson. 1996. The New Public Health: Health and Self in the Age of Risk. Sage Publications.

Lupton, D. and S. Chapman. 1995. "A healthy life-style might be the death of you: discourse on diet, cholesterol control and heart disease in the press and among the lay public" in Sociology of Health and Illness, 17(4): pp. 477-94.

MacCannell, Dean. 1976. "Staged Authenticity" The Tourist: A New Theory of the Leisure Class. New York: Schocken Books, pp 91-105

Martens, L. and A. Warde. 1997. "Urban Pleasure? On the Meaning of Eating Out in a Northern City" in P. Caplan (ed), Food, Health and Identity. London: Routledge, pp. $131-150$

Mason. 2006. 'Mixing methods in a qualitatively-driven way.' Qualitative Research. 6(1): pp 9-25.

Mennell, S. 1987. All Manners of Food. Eating and Taste in England and France from the Middle Ages to the Present. New York: Basil Blackwell.

- 1987. "On the Civilizing of Appetite" in Theory, Culture \& Society, 4(2): 373- 403.

Mintz, S. 1985. Sweetness and Power: The Place of Sugar in Modern History. New York: Viking.

Moltz, J. "Tasting an Imagined Thailand: Authenticity and Culinary Tourism in Thai Restaurants" in L. Long (ed.), Culinary Tourism. Lexington: The University Press of Kentucky, pp. 53-75

Parkin, W. and G. Craig. 2009. "Culture and the Politics of Alternative Food Networks" in Food, Culture \& Society. 12(1): pp. 78-103.

Pollen, M. 2007. In Defense of Food: An Eater's Manifesto. The Penguin Press.

Rose, N. 1996. Inventing Our Selves: Psychology, Power and Personhood. Cambridge: Cambridge University Press.

Roseberry, W. 1996. "The Rise of Yuppie Coffees and the Reimagination of Class in the United States" in American Anthropologist, 98(4): pp. 762-775.

Said, E. 1978. Orientalism. New York: Vintage. 
Schatzmann \& Strauss. 1973. Field Research; Strategies for a Natural Sociology. Englewood Cliff, NJ: Prentice-Hall.

Seale, C. 1999. The Quality of Qualitative Research. Sage Publications.

Simmel, G. 1997. "Sociology of the Meal" in D. Frisby and M. Featherstone (eds.), Simmel on Culture. London: Sage Publications, pp. 130-135.

Smith, A. \& J.B. MacKinnon. 2007. The 100 Mile Diet: A Year of Local Eating. Toronto: Random House.

Smith, D. 1999. "From Women's Standpoint to a Sociology for People." in J. AbuLugood, Sociology for the Twenty-first Century-Continuities and Cutting Edges. Chicago: University Press.

Snow \& Anderson. 1987. Identity work among the homeless: the verbal construction and avowal of personal identities. The American Journal of Sociology. 92: pp. 133671.

van Eijck, K. 2001. "Social Differentiation in Musical Taste Patterns" Social Forces 79: $1163-1184$.

Warde, A. 1997. Consumption, Food, and Taste: Culinary Antimonies and Commodity Culture. London: Sage.

Warde, A., Wright, D, \& Modesto Gayo-Cal. Understanding Cultural Omnivorousness: Or, the Myth of Cultural Omnivore. Cultural Sociology. July 2007. 1(2) pp. 143164.

Wilson, L. 2004. "Pass the Tofu, Please: Asian Food for Aging Baby Boomers" in L. Long (ed.) Culinary Tourism. Lexington: The University Press of Kentucky, pp. 245-267.

Winson, T. 2005. "Bringing political economy in to the debate on the obesity epidemic" in Agriculture and Human Values. 21(4): 299-312.

Zukin, S. 2004. Point of purchase: How shopping changed American culture. Routledge. 


\title{
CALL FOR INTERVIEW PARTICIPANTS
}

\section{Interested in urban food consumption? Been to The Green Door Restaurant?}

My name is Rebecca Warden and I am a graduate student in the department of Sociology and Anthropology at Carleton University. I am looking for individuals who have been to The Green Door Restaurant and would be interested in participating in research that $I$ am doing for my master's thesis.

The aim of my research is to understand the social value of a restaurant like The Green Door within a larger urban food system. I am hoping to interview 15 individuals who would be willing to share their experiences at The Green Door and talk about what this type of restaurant does or does not offer as a dining experience. Note that this project is in no way affiliated with The Green Door Restaurant, its owners, or its staff. This is not a business evaluation and the Green Door will not receive a report.

As a participant in my research project, you would participate in one semistructured interview that will last approximately 1 hour. Interviews will be conducted in person, with the specific time and location arranged to accommodate the schedules of respondents. With your permission, this interview will be recorded and transcribed. These recordings will be permanently deleted once the project has been completed (approximately May 2011).

Participants will be given pseudonyms in the transcription of interviews in order to maintain anonymity and ensure that no comments may be attributed to individual participants. There will not be any potential social risk for your participation in this research project because respondents will not be reliant on, or implicated with, the owners or staff of the restaurant. All views that you express during the interviews will be kept confidential. Also note that after participating in this interview, you may choose to withdraw from participating in this research project any time before January 17, 2011.

As a student researcher, I am supervised by Michael Mopas from Carleton University's Department of Sociology \& Anthropology and have had this project cleared by Carleton University's Research Ethics Committee. The contact information for my supervisor and Carleton University's Research Ethics Committee's Chair will be readily available to all participants.

Please respond via email to: rwarden@connect.carleton.ca. All responses would be greatly appreciated!

\author{
Rebecca Warden, MA Candidate \\ Department of Sociology \& Anthropology \\ Carleton University
}




\section{APPENDIX B}

\section{INTERVIEW SCRIPT}

\section{Primary Questions}

What makes food 'good'? What do you value in food?

What kind of an eater are you? How has this changed over time? How would you describe the food you eat on an everyday basis? The food your family eats? The food your partner eats?

\section{Why do you go to The Green Door?}

How would you describe food consumption in Ottawa? What do you perceive to be the strengths/weaknesses of our food system?

How would you describe The Green Door? Can you compare it to other food retailers you go to? Food? Décor? Staff? Energy?

\section{Secondary Questions}

How often do you go to The Green Door? Would you describe yourself as a 'regular'?

What do you get when you go to The Green Door? What are your favourite dishes? Are there any dishes you don't like? Why?

How do you choose food from the buffet? What is your selection strategy?

When did you first discover The Green Door? What can you remember of your first impressions? How have they changed?

How does the food at The Green Door compare to the dishes you prepare at home? Have you ever tried to replicate Green Door dishes?

Is there any particular moment that happened at The Green Door that stands out to you? A memorable visit? Meal? Conversation?

Is there anything that worries you about the food you eat? Do you have anxiety around eating? Your diet? Your body? 


\section{ANALYSIS FLOW CHART}
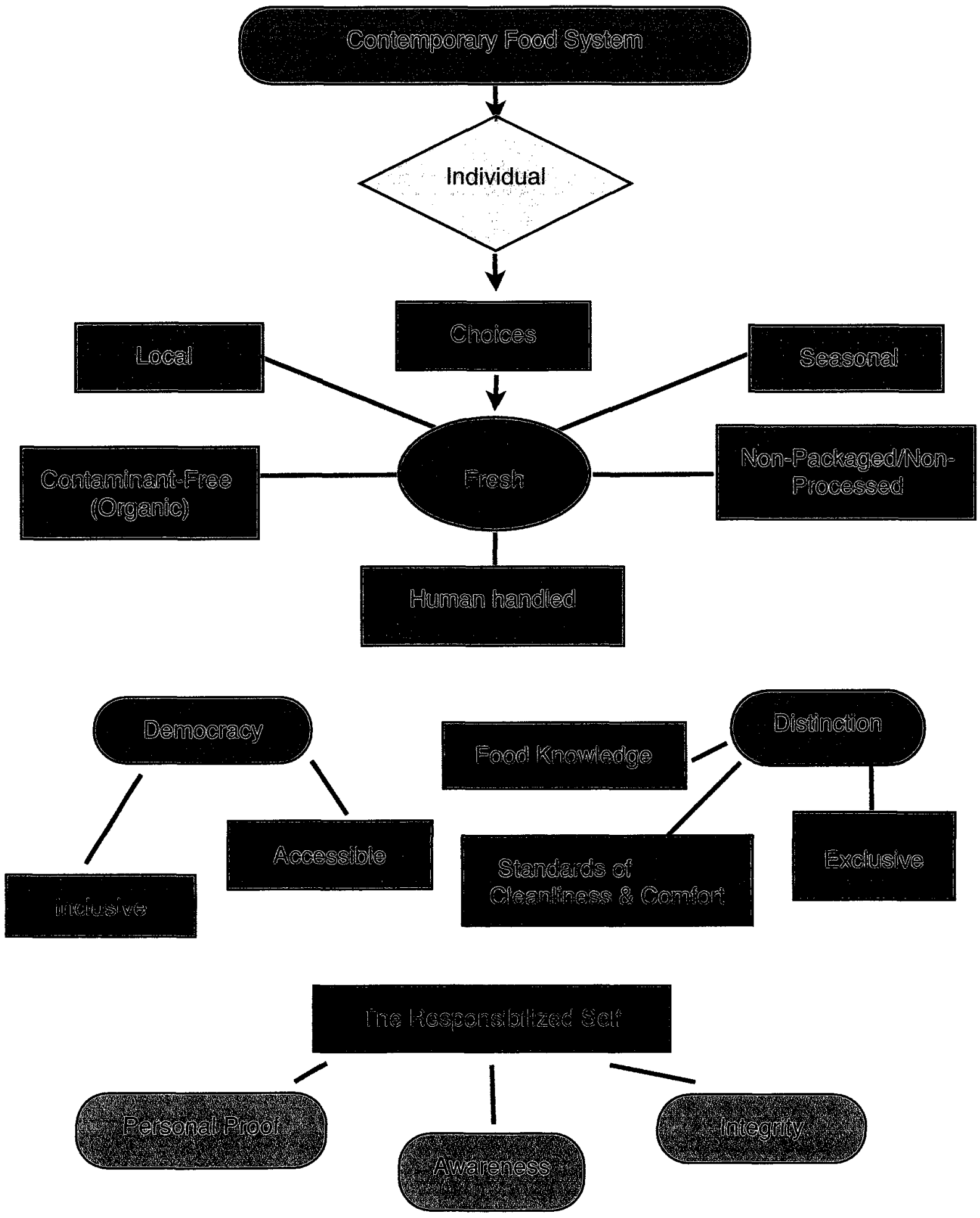